\title{
Stability of coassociative conical singularities
}

\author{
JASON D. LOTAY
}

\begin{abstract}
We study the stability of coassociative 4 -folds with conical singularities under perturbations of the ambient $\mathrm{G}_{2}$ structure by defining an integer invariant of a coassociative cone which we call the stability index. The stability index of a coassociative cone is determined by the spectrum of the curl operator acting on its link. We explicitly calculate the stability index for cones on group orbits. We also describe the stability index for cones fibred by 2-planes over algebraic curves using the degree and genus of the curve and the spectrum of the Laplacian on the link. Finally, we apply our results to construct the first known examples of coassociative 4-folds with conical singularities in compact manifolds with $\mathrm{G}_{2}$ holonomy.
\end{abstract}

\section{Introduction}

Coassociative 4-folds are calibrated, hence minimal, submanifolds of 7-manifolds with $\mathrm{G}_{2}$ structures, first defined in [10]. Of particular interest are coassociative 4 -folds in manifolds with $\mathrm{G}_{2}$ holonomy, of which few examples are known. Coassociative submanifolds with conical singularities have been previously studied by the author in $[25,26]$, building upon the work on special Lagrangian submanifolds with conical singularities by Joyce in $[14-18]$.

We continue to generalize the work of Joyce to the coassociative setting by defining the notion of stability index for coassociative cones. The stability index is a non-negative integer invariant for a coassociative cone, the vanishing of which guarantees that coassociative 4-folds with a singularity modelled on that cone will have a smooth moduli space of deformations: in particular, they are stable under small perturbations of the $\mathrm{G}_{2}$ structure on the 7-manifold.

We calculate the stability index for certain types of coassociative cones and use our results to construct the first examples of coassociative 4-folds with conical singularities in compact manifolds with $\mathrm{G}_{2}$ holonomy. This is 
an essential step in the proposed construction given in [21] of coassociative fibrations of compact $\mathrm{G}_{2}$ manifolds.

\subsection{Motivation}

Even though there has been a wealth of research devoted to calibrated submanifolds with conical singularities in manifolds with special holonomy, particularly in [14-18] and [25, 26], there were no known examples of such submanifolds. Given this well-developed theory, we were motivated to construct coassociative examples. As far as the author is aware, it is unknown whether there are special Lagrangian $m$-folds with conical singularities in Calabi-Yau $m$-folds for $m \geq 3$.

The stability index of a special Lagrangian cone is defined in [15] in terms of the spectrum of the Laplacian on the link, and is calculated for cones over flat tori originally given in [10, Section III]. The stability index for special Lagrangian cones over certain homogeneous spaces is calculated in [34] and Haskins [11] shows that the only stable special Lagrangian $T^{2}$-cone is the cone over the flat torus.

In contrast, the stability index for a coassociative cone is determined by the spectrum of the curl operator acting on 1-forms on the 3-dimensional link of the cone. Although still a natural geometric object, there is relatively little material on the spectrum of the curl operator in the literature, and it is certainly less straightforward to analyse than the spectrum of the Laplacian which has received so much attention. We are thus required to undertake fundamental elementary calculations to describe the stability index even in simple cases.

Eigenforms for the curl operator naturally define contact structures and are dual to certain Beltrami fields, which are important in hydrodynamics. Beltrami fields are also equivalent to Reeb vector fields by the work in [8], so are of particular interest in contact geometry. We hope therefore that our spectral calculations may be of wider benefit.

Motivated by the SYZ conjecture (see [38]), one would hope to construct coassociative fibrations of compact manifolds with $\mathrm{G}_{2}$ holonomy. An elementary argument shows that some of the fibres must necessarily be singular, so it is natural to assume that they have the simplest type of singularity, namely conical singularities. As already mentioned, the stability results in this paper are essential for the proposed construction of a coassociative $K 3$ fibration in [21]. Moreover, our results will almost certainly be useful for any other construction of a coassociative fibration with conically singular fibres. 


\subsection{Summary}

We begin in Section 2 with the basic definitions we need, including concepts from calibrated geometry and Geometric Measure Theory. We also discuss the relationship between complex and coassociative geometry.

In Section 3 we mainly review the material in [25], defining coassociative 4-folds with conical singularities and describing their deformation theory. Our definition of coassociative conical singularities (Definition 3.2) on the face of it looks rather strong. However, we prove the following regularity result, generalizing results in $[15,37]$, which shows that the definition is more applicable than it appears.

Theorem 1.1. If a coassociative integral current has a multiplicity one, Jacobi integrable tangent cone with isolated singularity at an interior point $p$, then it has a conical singularity at $p$.

Remarks. The link of a coassociative cone is a Lagrangian (or totally real) submanifold of the nearly Kähler 6 -sphere $\mathcal{S}^{6}$. A coassociative cone is Jacobi integrable if every infinitesimal variation of its link as a Lagrangian in $\mathcal{S}^{6}$ is integrable.

In Section 4, we begin by briefly reviewing the known examples of coassociative cones. Then, by refining some of the results in [25], we define the notion of $\mathcal{C}$-stability index for a coassociative cone $C$ in a family $\mathcal{C}$. We simply call the $\mathcal{C}$-stability index of $C$ the stability index when $\mathcal{C}$ is the family of cones generated by $\mathrm{G}_{2}$ transformations and translations of $C$.

Sections 5 and 6 are devoted to calculating the stability index for certain coassociative cones. In Section 5, we analyse the curl operator acting on Berger 3-spheres and their quotients by finite groups, and thus determine the stability index for all homogeneous coassociative cones. We deduce the following.

Theorem 1.2. The only stable homogeneous coassociative cones are coassociative 4-planes and the $\mathrm{Sp}(1)$-invariant coassociative cone given in Example 4.2.

Remark. The cone in Example 4.2 was originally constructed in [22, Section 7] and identified as coassociative in [10, Section IV]. 
In Section 6 we study coassociative cones which are fibred by 2-planes over algebraic curves: either holomorphic curves in $\mathbb{C P}^{2}$, where the corresponding coassociative cone is complex, or null-torsion pseudoholomorphic curves in $\mathcal{S}^{6}$. In both cases, we express the stability index in terms of the spectrum of the Laplacian acting on functions on the link and algebrogeometric data from the curve. These results allow us to examine the behaviour of the stability index under deformations of these types of coassociative cones.

Finally, in Section 7, after discussing the construction in [20] of compact manifolds with $\mathrm{G}_{2}$ holonomy, we apply the results of Sections 5 and 6 to prove the following.

Theorem 1.3. Given a pair of maximal deformation families of Fano 3folds, one can construct a one-parameter family of compact manifolds with $\mathrm{G}_{2}$ holonomy, which contain coassociative K3 surfaces with conical singularities.

Remark. The key ingredients in the proof are Theorem 1.1, the $\mathcal{C}$-stability index for a homogeneous complex cone $C$ in a deformation family $\mathcal{C}$ and the invariance of the $\mathcal{C}$-stability index under deformations of $C$ in $\mathcal{C}$.

Given the proof of Theorem 1.3, it is clear that this result will naturally extend to give coassociative 4-folds with conical singularities in the compact holonomy $\mathrm{G}_{2}$ manifolds constructed by Corti et al. [6], where one replaces Fano 3 -folds by a more general class of complex 3 -folds.

\section{Notes.}

(a) Manifolds are taken to be non-singular and submanifolds to be embedded, for convenience, unless stated otherwise.

(b) We use the convention that $\mathbb{N}=\{0,1,2, \ldots\}$ and $\mathbb{Z}^{+}=\mathbb{N} \backslash\{0\}$.

\section{Coassociative 4 -folds}

In this section, we cover all of the basic definitions and theory we need.

\subsection{Calibrated geometry and Geometric Measure Theory}

We will need some general theory from calibrated geometry later, so we start with the following definition. 
Definition 2.1. Let $(M, g)$ be a Riemannian manifold. An oriented tangent $m$-plane $W$ on $M$ is an oriented $m$-dimensional vector subspace $W$ of $T_{x} M$, for some $x$ in $M$. Given an oriented tangent $m$-plane $W$ on $M,\left.g\right|_{W}$ is a Euclidean metric on $W$ and hence, using $\left.g\right|_{W}$ and the orientation on $W$, there is a natural volume form, $\operatorname{vol}_{W}$, which is an $m$-form on $W$.

A closed $m$-form $\phi$ on $M$ is a calibration on $M$ if $\left.\phi\right|_{W} \leq \operatorname{vol}_{W}$ for all oriented tangent $m$-planes $W$ on $M$, where $\left.\phi\right|_{W}=\kappa \cdot \operatorname{vol}_{W}$ for some $\kappa \in \mathbb{R}$, so $\left.\phi\right|_{W} \leq \operatorname{vol}_{W}$ if $\kappa \leq 1$. An oriented $m$-dimensional submanifold $S$ of $M$ is a calibrated submanifold or $\phi$-submanifold if $\left.\phi\right|_{S}=\operatorname{vol}_{S}$.

We shall need some ideas from Geometric Measure Theory. A good introduction to this theory, which we only need in a superficial way, is given in $[32]$.

Definition 2.2. Let $(M, g)$ be a complete Riemannian manifold and let $\mathcal{H}^{m}$ be $m$-dimensional Hausdorff measure on $M$. By [32, Proposition 3.11], one can define an $\mathcal{H}^{m}$-measurable subset $S$ of $M$ to be an $m$-dimensional rectifiable set if $S$ has finite $\mathcal{H}^{m}$-measure and $\mathcal{H}^{m}$-almost all of $S$ is covered by the disjoint union of a countable number of compact $C^{1}$-submanifolds of $M$. Let $\operatorname{vol}(S)$ be the $\mathcal{H}^{m}$-measure of $S$. By [32, Proposition 3.12], an $m$-dimensional rectifiable set $S$ in $M$ has a well-defined $m$-dimensional tangent plane $\mathcal{H}^{m}$-almost everywhere, and so is orientable almost everywhere. If $S$ is an oriented rectifiable set, let $\mathbf{s}(x)$ be the unit $m$-vector to $S$ at $x$ given by the choice of orientation, when this is well-defined.

Let $\mathcal{D}^{m}(M)$ be the space of smooth compactly supported $m$-forms on $M$. An $m$-dimensional current on $M$ is an element of the dual space $\mathcal{C}^{m}(M)=$ $\mathcal{D}^{m}(M)^{*}$ and we define the support of $T \in \mathcal{C}^{m}(M)$, supp $T$, to be the smallest closed set in $M$ such that, for any $\xi \in \mathcal{D}^{m}(M)$, supp $\xi \cap \operatorname{supp} T=\emptyset$ implies that $T(\xi)=0$. We define the boundary $\partial T \in \mathcal{C}^{m-1}(M)$ of $T \in \mathcal{C}^{m}(M)$ via the formula $\partial T(\xi)=T(\mathrm{~d} \xi)$ for $\xi \in \mathcal{D}^{m-1}(M)$. We also define the interior $T^{\circ}$ of $T$ to be the set $\operatorname{supp} T \backslash \operatorname{supp} \partial T$.

Given an $m$-dimensional oriented rectifiable set $S$ and a function $\nu$ : $S \rightarrow \mathbb{Z}^{+}$such that $\int_{S} \nu(x) \mathrm{d} \mathcal{H}^{m}<\infty$, we define an associated element $T_{S}$ of $\mathcal{C}^{m}(M)$ via

$$
T_{S}(\xi)=\int_{S} \mathbf{s}(x) \cdot \xi(x) \nu(x) \mathrm{d} \mathcal{H}^{m} .
$$

If supp $T_{S}$ is compact, we say that $T_{S}$ (or simply $S$, since $T_{S}$ is defined by $S$ ) is an $m$-dimensional rectifiable current and we denote the set of $m$-dimensional rectifiable currents by $\mathcal{R}^{m}(M)$. We also let $\mathcal{I}^{m}(M)=\left\{S \in \mathcal{R}^{m}(M): \partial S \in\right.$ $\left.\mathcal{R}^{m-1}(M)\right\}$ be the set of $m$-dimensional integral currents. 
Finally, we define the set of $m$-dimensional locally integral currents by

$$
\mathcal{I}_{\mathrm{loc}}^{m}(M)=\left\{T \in \mathcal{C}^{m}(M): \forall x \in M \exists S \in \mathcal{I}^{m}(M) \text { with } x \notin \operatorname{supp}(T-S)\right\}
$$

and similarly define $\mathcal{R}_{\mathrm{loc}}^{m}(M)$.

The idea behind a rectifiable current is to generalize the notion of a compact $C^{1}$-submanifold with boundary to include multiplicities (given by the function $\nu$ ) and to allow for singular behaviour. Since the boundary of a rectifiable current may be very badly behaved, we often require that the rectifiable current be integral. As we shall need to deal with planes and cones which are definitely not compact, we expand our notation to include the "local" versions of the integral and rectifiable currents.

The majority of this paper will be dedicated to the study of cones, so we make some formal definitions for convenience.

Definition 2.3. Recall the notation of Definition 2.2, let $W$ be a normed vector space and let $\mathcal{S}(W)$ be the unit sphere in $W$ with respect to the norm. An element $C \in \mathcal{R}_{\text {loc }}^{m}(W)$ is a cone in $W$ if $t C=C$ for all $t>0$, and we call $C \cap \mathcal{S}(W)$ the link of $C$.

We formally define convergence in the space of currents as follows.

Definition 2.4. Recall the notation of Definition 2.2. We say that a sequence $\left(S_{j}\right)$ in $\mathcal{R}_{\text {loc }}^{m}(M)$ converges to $S \in \mathcal{R}_{\text {loc }}^{m}(M)$ if $S_{j} \rightarrow S$ in the weak topology in $\mathcal{C}^{m}(M)$; that is,

$$
S_{j} \rightarrow S \text { if and only if } \int_{S_{j}} \xi \rightarrow \int_{S} \xi \text { as } j \rightarrow \infty,
$$

for all $\xi \in \mathcal{D}^{m}(M)$, where integration is carried out with respect to $\mathcal{H}^{m}$ and includes multiplicities.

In the seminal work on calibrated geometry [10], the relationship between calibrated geometry and Geometric Measure Theory is discussed at length. We note some of the observations originally presented there.

Definition 2.5. Let $(M, g)$ be a complete Riemannian manifold, let $\phi$ be an $m$-form which is a calibration on $M$ and recall the notation of Definition 2.2. By the work in [10], we can define $S \in \mathcal{I}^{m}(M)$ to be an integral $\phi$-current if $S$ is calibrated with respect to $\phi$; that is, $\int_{S} \phi=\operatorname{vol}(S)$. Then integral $\phi$-currents are volume-minimizing in their homology class. We can also define a locally integral $\phi$-current in $M$ in the obvious manner. 
One of the key ideas in Geometric Measure Theory is the concept of a tangent cone, which we now define.

Definition 2.6. Let $(M, g)$ be a complete Riemannian $n$-manifold and recall the notation of Definition 2.2. Let $S \in \mathcal{R}_{\text {loc }}^{m}(M)$ and let $x \in S^{\circ}$. Choose a diffeomorphism $v: V \rightarrow B$, where $B$ is an open neighbourhood of the origin in $\mathbb{R}^{n}$ and $V$ is an open neighbourhood of $x$ in $M$. Let $U=V \cap S$ and let $\Upsilon=\left.\mathrm{d} v\right|_{x}$, which is an isomorphism between $T_{x} M$ and $\mathbb{R}^{n}$. A tangent cone for $S$ at $x$ is a cone $C$ in $T_{x} M$ such that there exists a strictly decreasing positive sequence $\left(r_{j}\right)$, converging to zero as $j \rightarrow \infty$, such that

$$
r_{j}^{-1} v(U \backslash\{x\}) \rightarrow \Upsilon(C) \quad \text { as } j \rightarrow \infty
$$

in the sense of Definition 2.4.

In Geometric Measure Theory there are two notions of "tangent cone": one is a set and the other is a current. We have defined a current in Definition 2.6 and so it is, strictly speaking, an oriented tangent cone in the sense of Geometric Measure Theory. Oriented tangent cones can be defined for more general currents than locally rectifiable ones, but then one only requires a weaker form of convergence in the definition.

We conclude with an important result that follows from $[19$, Theorem 4.4.4].

Proposition 2.7. Let $\phi$ be a calibration on a complete Riemannian manifold $M$ and let $S$ be an integral $\phi$-current in $M$. There exists a tangent cone to $S$ at each $x \in S^{\circ}$, and it is a locally integral $\left.\phi\right|_{x^{-c u r r e n t}}$ in $T_{x} M$.

\subsection{Calibrated geometry in $\mathbb{R}^{7}$}

We define coassociative 4 -folds in $\mathbb{R}^{7}$ by introducing a distinguished 3 -form.

Definition 2.8. Let $\left(x_{1}, \ldots, x_{7}\right)$ be coordinates on $\mathbb{R}^{7}$ and write $\mathrm{d} \mathbf{x}_{i j \ldots k}$ for the form $\mathrm{d} x_{i} \wedge \mathrm{d} x_{j} \wedge \cdots \wedge \mathrm{d} x_{k}$. Define a 3 -form $\varphi_{0}$ on $\mathbb{R}^{7}$ by:

$$
\varphi_{0}=\mathrm{d} \mathbf{x}_{123}+\mathrm{d} \mathbf{x}_{145}+\mathrm{d} \mathbf{x}_{167}+\mathrm{d} \mathbf{x}_{246}-\mathrm{d} \mathbf{x}_{257}-\mathrm{d} \mathbf{x}_{347}-\mathrm{d} \mathbf{x}_{356}
$$

The Hodge dual of $\varphi_{0}$ is a 4-form given by:

$$
* \varphi_{0}=\mathrm{d} \mathbf{x}_{4567}+\mathrm{d} \mathbf{x}_{2367}+\mathrm{d} \mathbf{x}_{2345}+\mathrm{d} \mathbf{x}_{1357}-\mathrm{d} \mathbf{x}_{1346}-\mathrm{d} \mathbf{x}_{1256}-\mathrm{d} \mathbf{x}_{1247}
$$


The forms $\varphi_{0}$ and $* \varphi_{0}$ are calibrations by [10, Theorems IV.1.4 and IV.1.16]. Submanifolds calibrated with respect to $\varphi_{0}$ and $* \varphi_{0}$ are called associative 3-folds and coassociative 4-folds respectively. We can also characterize the coassociative 4 -folds as the oriented 4 -dimensional submanifolds $N$ in $\mathbb{R}^{7}$ satisfying $\left.\varphi_{0}\right|_{N} \equiv 0$, oriented such that $\left.* \varphi_{0}\right|_{N}>0$, by [10, Proposition IV.4.5 and Theorem IV.4.6].

Remark. The form $\varphi_{0}$ is sometimes called the " $\mathrm{G}_{2} 3$-form" because the exceptional Lie group $\mathrm{G}_{2}$ is the stabilizer of $\varphi_{0}$ in $\mathrm{GL}(7, \mathbb{R})$.

A straightforward calculation yields the following lemma.

Lemma 2.9. Identify $\mathbb{R}^{7}$ with $\mathbb{R} \oplus \mathbb{C}^{3}$ so that $x_{1}$ is the coordinate on $\mathbb{R}$ and $z_{1}=x_{2}+i x_{3}, z_{2}=x_{4}+i x_{5}$ and $z_{3}=x_{6}+i x_{7}$ are coordinates on $\mathbb{C}^{3}$. If $\omega_{0}$ and $\Omega_{0}$ are the standard Kähler and holomorphic forms on $\mathbb{C}^{3}$, then:

$$
\begin{aligned}
\varphi_{0} & =\mathrm{d} x_{1} \wedge \omega_{0}+\operatorname{Re} \Omega_{0}, \\
* \varphi_{0} & =\frac{1}{2} \omega_{0} \wedge \omega_{0}-\mathrm{d} x_{1} \wedge \operatorname{Im} \Omega_{0},
\end{aligned}
$$

where $\varphi_{0}$ and $* \varphi_{0}$ are given in (2.1) and (2.2).

Since $\operatorname{Re} \Omega_{0}$ and $\operatorname{Im} \Omega_{0}$ are both calibrations on $\mathbb{C}^{3}$ we have the following definition, again due to Harvey and Lawson [10].

Definition 2.10. Let $\left(z_{1}, \ldots, z_{m}\right)$ be coordinates on $\mathbb{C}^{m}$ and let $\omega_{0}$ and $\Omega_{0}$ be the Kähler and holomorphic volume forms on $\mathbb{C}^{m}$. Then $\cos \theta \operatorname{Re} \Omega_{0}+$ $\sin \theta \operatorname{Im} \Omega_{0}$ is a calibration on $\mathbb{C}^{m}$ for all real constants $\theta$, and its corresponding calibrated submanifolds are real $m$-dimensional submanifolds of $\mathbb{C}^{m}$ called special Lagrangian $m$-folds (with phase $e^{i \theta}$ ). Moreover, special Lagrangian $m$-folds with phase $e^{i \theta}$ are the oriented real $m$-dimensional submanifolds $L$ of $\mathbb{C}^{m}$ such that $\left.\omega_{0}\right|_{L} \equiv 0$ and $\left.\left(\sin \theta \operatorname{Re} \Omega_{0}-\cos \theta \operatorname{Im} \Omega_{0}\right)\right|_{L} \equiv 0$, up to a choice of orientation.

Examination of (2.3) and (2.4) immediately yields the following elementary result.

Corollary 2.11. In the notation of Lemma 2.9, $\mathbb{R} \times L \subseteq \mathbb{R} \oplus \mathbb{C}^{3}$ and $N \subseteq$ $\mathbb{C}^{3}$ are coassociative in $\mathbb{R}^{7}$ if and only if $L$ is a special Lagrangian 3-fold with phase $-i$ and $N$ is a complex surface in $\mathbb{C}^{3}$ respectively. 
Since we will be concerned with coassociative cones, we make the following convenient definition.

Definition 2.12. The 6-sphere $\mathcal{S}^{6}$ inherits a nearly Kähler structure from the standard $\mathrm{G}_{2}$ structure on $\mathbb{R}^{7}$. In particular, if $r$ is the radial coordinate and $\mathbf{e}_{r}$ is the radial vector field on $\mathbb{R}^{7}, \omega=\left.\left(\mathbf{e}_{r} \cdot \varphi_{0}\right)\right|_{r=1}$ is a non-degenerate 2 -form on $\mathcal{S}^{6}$ which is not closed. Using this 2 -form and the round metric $g$ on $\mathcal{S}^{6}$ we can define an almost complex structure $J$ by $\omega(x, y)=g(J x, y)$ for tangent vectors $x, y$. The almost complex structure $J$ is not integrable.

An oriented 3-dimensional submanifold $L \subseteq \mathcal{S}^{6}$ is the link of a coassociative cone if and only if $\left.\omega\right|_{L} \equiv 0$. Thus, we say that the link of a coassociative cone in $\mathbb{R}^{7}$ is a Lagrangian submanifold of $\mathcal{S}^{6}$.

An oriented surface $\Sigma \subseteq \mathcal{S}^{6}$ is a pseudoholomorphic curve if and only if $\left.\omega\right|_{\Sigma}=\operatorname{vol}_{\Sigma}$ or, equivalently, if $J\left(T_{\sigma} \Sigma\right)=T_{\sigma} \Sigma$ for all $\sigma \in \Sigma$. Note that $\Sigma$ is the link of an associative cone if and only if $\Sigma$ is a pseudoholomorphic curve.

By Corollary 2.11, any complex 2-dimensional cone in $\mathbb{C}^{3}$ is coassociative in $\mathbb{R} \oplus \mathbb{C}^{3} \cong \mathbb{R}^{7}$. Thus, the Hopf lift of any holomorphic curve in $\mathbb{C P}^{2}$ to a totally geodesic $\mathcal{S}^{5}$ in $\mathcal{S}^{6}$ is Lagrangian. Since special Lagrangian 3 -folds in $\mathbb{C}^{3}$ are associative in $\mathbb{R} \oplus \mathbb{C}^{3} \cong \mathbb{R}^{7}$, minimal Legendrian surfaces in $\mathcal{S}^{5}$, which are the links of special Lagrangian cones in $\mathbb{C}^{3}$, give examples of pseudoholomorphic curves in $\mathcal{S}^{6}$.

\section{3. $\mathrm{G}_{2}$ structures}

So that we may define coassociative submanifolds of more general 7-manifolds, we make the following definition.

Definition 2.13. Let $M$ be an oriented 7 -manifold and recall the 3 -form $\varphi_{0}$ on $\mathbb{R}^{7}$ given in (2.1). For each $x \in M$ there exists an orientation preserving isomorphism $\iota_{x}: T_{x} M \rightarrow \mathbb{R}^{7}$. Since $\operatorname{dim} \mathrm{G}_{2}=14, \operatorname{dim} \mathrm{GL}_{+}\left(T_{x} M\right)=49$ and $\operatorname{dim} \Lambda^{3} T_{x}^{*} M=35$, the $\mathrm{GL}_{+}\left(T_{x} M\right)$ orbit of $\iota_{x}^{*}\left(\varphi_{0}\right)$ in $\Lambda^{3} T_{x}^{*} M$, denoted $\Lambda_{+}^{3} T_{x}^{*} M$, is open. A 3 -form $\varphi$ on $M$ is positive if $\left.\varphi\right|_{x} \in \Lambda_{+}^{3} T_{x}^{*} M$ for all $x \in M$. Denote the bundle of positive 3 -forms by $\Lambda_{+}^{3} T^{*} M$.

A positive 3 -form is identified with the $\mathrm{G}_{2} 3$-form $\varphi_{0}$ on $\mathbb{R}^{7}$ at each point in $M$. Therefore, to each positive 3 -form $\varphi$ we can uniquely associate a 4 -form $*_{\varphi} \varphi$ and a metric $g_{\varphi}$ on $M$ such that the triple $\left(\varphi, *_{\varphi} \varphi, g_{\varphi}\right)$ corresponds to $\left(\varphi_{0}, * \varphi_{0}, g_{0}\right)$ at each point. Note that since the metric $g_{\varphi}$ depends on $\varphi$, the Hodge star $*_{\varphi}$ depends on $\varphi$ also. This leads us to our next definition. 
Definition 2.14. Let $M$ be an oriented 7-manifold and $\varphi \in C^{\infty}\left(\Lambda_{+}^{3} T^{*} M\right)$. If $g_{\varphi}$ is the metric associated with $\varphi$, we call $\left(\varphi, g_{\varphi}\right)$ a $\mathrm{G}_{2}$ structure on $M$. If $\varphi$ is closed (or $*_{\varphi} \varphi$ is closed) then $\left(\varphi, g_{\varphi}\right)$ is a closed (or coclosed) $\mathrm{G}_{2}$ structure. A closed and coclosed $\mathrm{G}_{2}$ structure is called torsion-free.

Our choice of notation here agrees with [5].

Remark. By [35, Lemma 11.5], $\left(\varphi, g_{\varphi}\right)$ is a torsion-free $\mathrm{G}_{2}$ structure on $M$ if and only if the holonomy of $g_{\varphi}$ is contained in $\mathrm{G}_{2}$.

Definition 2.15. Let $M$ be an oriented 7-manifold with a $\mathrm{G}_{2}$ structure $\left(\varphi, g_{\varphi}\right)$, denoted $\left(M, \varphi, g_{\varphi}\right)$. If $\left(\varphi, g_{\varphi}\right)$ is closed, we say that $\left(M, \varphi, g_{\varphi}\right)$ is an almost $\mathrm{G}_{2}$ manifold. If $\left(\varphi, g_{\varphi}\right)$ is torsion-free, we call $\left(M, \varphi, g_{\varphi}\right)$ a $\mathrm{G}_{2}$ manifold.

Note. By [19, Proposition 11.2.1], the metric $g_{\varphi}$ on a compact $\mathrm{G}_{2}$ manifold $M$ has $\mathrm{G}_{2}$ holonomy if and only if the fundamental group $\pi_{1}(M)$ is finite.

We are now able to complete our definitions regarding coassociative 4-folds.

Definition 2.16. A 4-dimensional submanifold $N$ of $\left(M, \varphi, g_{\varphi}\right)$ is coassociative if and only if $\left.\varphi\right|_{N} \equiv 0$ and $\left.*_{\varphi} \varphi\right|_{N}>0$.

Note. Though we may define coassociative 4 -folds with respect to any $\mathrm{G}_{2}$ structure, for deformation theory and related results to hold we need it to be closed. Therefore, we shall work with almost $\mathrm{G}_{2}$ manifolds for greatest useful generality.

The next result, [31, cf. Proposition 4.2], is invaluable in describing the deformation theory of coassociative 4 -folds.

Proposition 2.17. Let $N$ be a coassociative 4-fold in an almost $\mathrm{G}_{2}$ manifold $\left(M, \varphi, g_{\varphi}\right)$. There is an isometric isomorphism between the normal bundle $\nu(N)$ of $N$ in $M$ and $\left(\Lambda_{+}^{2}\right)_{\left.g_{\varphi}\right|_{N}} T^{*} N$ given by $\left.\jmath_{N}: v \mapsto(v\lrcorner \varphi\right)\left.\right|_{T N}$. Thus, infinitesimal coassociative deformations of $N$ are governed by closed self-dual 2-forms on $N$.

Remarks. From Proposition 2.17 and some further analysis, one may deduce as in [19, Theorem 12.3.4], by following [31, Theorem 4.5], that the moduli space of deformations of a compact coassociative 4 -fold $N$ in 
an almost $\mathrm{G}_{2}$ manifold is a manifold of dimension $b_{+}^{2}(N)$. The author in [25] adapted this deformation theory result to the situation where $N$ has conical singularities, which will be invaluable for the study in this article. The relevant material in [25] will be the focus of Section 3.

We shall also be briefly concerned with $\mathrm{SU}(3)$ structures, so they form the subject of the next definition.

Definition 2.18. On $\mathbb{C}^{3}$, let $g_{0}, \omega_{0}$ and $\Omega_{0}$ denote the standard Euclidean metric, Kähler form and holomorphic volume form respectively. Let $(Y, J, g, \omega)$ be an almost Hermitian 6-manifold; that is, $g$ is a Riemannian metric on the almost complex 6 -manifold $Y, J$ is an almost complex structure preserved by $g$ and $\omega$ is the associated (non-degenerate) (1,1)-form on $M$. An $\mathrm{SU}(3)$ structure on $Y$ is a choice of nowhere vanishing $(3,0)$-form $\Omega$ on $Y$ such that, for all $y \in Y$, there exists an orientation preserving isomorphism $\iota_{y}: T_{y} Y \rightarrow \mathbb{C}^{3}$ satisfying $\iota_{y}^{*}\left(g_{0}\right)=\left.g\right|_{y}, \iota_{y}^{*}\left(\omega_{0}\right)=\left.\omega\right|_{y}$ and $\iota_{y}^{*}\left(\Omega_{0}\right)=\left.\Omega\right|_{y}$.

If $(Y, J, g, \omega, \Omega)$ is an almost Hermitian 6-manifold endowed with an $\mathrm{SU}(3)$ structure, the product 7 -manifold $M=\mathbb{R} \times Y\left(\right.$ or $\mathcal{S}^{1} \times Y$ ) has a "product" $\mathrm{G}_{2}$ structure given by $\varphi=\mathrm{d} x \wedge \omega+\operatorname{Re} \Omega$ and $g_{\varphi}=\mathrm{d} x^{2}+g$, where $x$ is the coordinate on $\mathbb{R}$ or $\mathcal{S}^{1}$, by [19, Proposition 11.1.9]. Moreover, $*_{\varphi} \varphi=\frac{1}{2} \omega \wedge \omega-\mathrm{d} x \wedge \operatorname{Im} \Omega$.

Notes. If $(Y, g, J, \omega)$ is a compact Kähler 3 -manifold and $\Omega$ is a nowhere vanishing holomorphic $(3,0)$-form on $Y$, then $(Y, g, J, \omega, \Omega)$ is called an almost Calabi-Yau 3-fold. If, in addition, $\omega^{3}=\frac{3 i}{4} \Omega \wedge \bar{\Omega}$ then the $\mathrm{SU}(3)$ structure is torsion-free and $(Y, J, g, \omega, \Omega)$ is called a Calabi-Yau 3-fold. These are the natural "SU(3) analogues" of the manifolds defined in Definition 2.15. In particular, the Calabi-Yau condition is equivalent to saying that the compact Kähler manifold has metric $g$ with holonomy contained in $\mathrm{SU}(3)$.

\section{Conical singularities}

In this section, we review some of the theory of conical singularities of coassociative 4-folds as described in [25]. We also prove an important new result that shows that singular coassociative integral currents with particularly "nice" tangent cones have conical singularities.

\subsection{Coassociative 4 -folds with conical singularities}

We first define a preferred choice of local coordinates on an almost $\mathrm{G}_{2}$ manifold near a finite set of points, which is an analogue of one given for almost 
Calabi-Yau manifolds in $\left[15\right.$, Definition 3.6]. We let $B(0 ; \delta) \subseteq \mathbb{R}^{7}$ denote the open ball about 0 with radius $\delta>0$.

Definition 3.1. Let $\left(M, \varphi, g_{\varphi}\right)$ be an almost $\mathrm{G}_{2}$ manifold and let $z_{1}, \ldots$, $z_{s} \in M$ be distinct points. There exist a constant $\epsilon_{M} \in(0,1)$, an open set $V_{i} \ni z_{i}$ in $M$ with $V_{i} \cap V_{j}=\emptyset$ for $j \neq i$ and a diffeomorphism $\chi_{i}: B\left(0 ; \epsilon_{M}\right) \rightarrow$ $V_{i}$ with $\chi_{i}(0)=z_{i}$, for $i=1, \ldots, s$, such that $\zeta_{i}=\left.\mathrm{d} \chi_{i}\right|_{0}: \mathbb{R}^{7} \rightarrow T_{z_{i}} M$ is an isomorphism identifying the standard $\mathrm{G}_{2}$ structure $\left(\varphi_{0}, g_{0}\right)$ on $\mathbb{R}^{7}$ with the pair $\left(\left.\varphi\right|_{z_{i}},\left.g_{\varphi}\right|_{z_{i}}\right)$. We call the set $\left\{\chi_{i}: B\left(0 ; \epsilon_{M}\right) \rightarrow V_{i}: i=1, \ldots, s\right\}$ a $\mathrm{G}_{2}$ coordinate system near $z_{1}, \ldots, z_{s}$.

We say that two $\mathrm{G}_{2}$ coordinate systems near $z_{1}, \ldots, z_{s}$, with maps $\chi_{i}$ and $\tilde{\chi}_{i}$ for $i=1, \ldots, s$, respectively, are equivalent if $\left.\mathrm{d} \tilde{\chi}_{i}\right|_{0}=\left.\mathrm{d} \chi_{i}\right|_{0}=\zeta_{i}$ for all $i$.

Definition 3.2. Let $\left(M, \varphi, g_{\varphi}\right)$ be an almost $\mathrm{G}_{2}$ manifold, let $N \subseteq M$ be compact and connected and let $z_{1}, \ldots, z_{s} \in N$ be distinct. We also let $\left\{\chi_{i}: B\left(0 ; \epsilon_{M}\right) \rightarrow V_{i}: i=1, \ldots, s\right\}$ be a $\mathrm{G}_{2}$ coordinate system near $z_{1}, \ldots, z_{s}$, as in Definition 3.1. We say that $N$ is a 4 -fold in $M$ with conical singularities at $z_{1}, \ldots, z_{s}$ with rate $\mu$, denoted a $C S$ 4-fold, if $\hat{N}=N \backslash\left\{z_{1}, \ldots, z_{s}\right\}$ is a (non-singular) 4-dimensional submanifold of $M$ and there exist constants $0<\epsilon<\epsilon_{M}$ and $\mu \in(1,2)$, a compact 3-dimensional Riemannian submanifold $\left(L_{i}, h_{i}\right)$ of $\mathcal{S}^{6} \subseteq \mathbb{R}^{7}$, where $h_{i}$ is the restriction of the round metric on $\mathcal{S}^{6}$ to $L_{i}$, an open set $U_{i} \ni z_{i}$ in $N$ with $U_{i} \subseteq V_{i}$ and a smooth map $\Phi_{i}:(0, \epsilon) \times L_{i} \rightarrow B\left(0 ; \epsilon_{M}\right) \subseteq \mathbb{R}^{7}$, for $i=1, \ldots, s$, such that $\Psi_{i}=\chi_{i} \circ \Phi_{i}:$ $(0, \epsilon) \times L_{i} \rightarrow U_{i} \backslash\left\{z_{i}\right\}$ is a diffeomorphism, and $\Phi_{i}$ satisfies

$$
\Phi_{i}\left(r_{i}, x_{i}\right)-\iota_{i}\left(r_{i}, x_{i}\right) \in\left(T_{r_{i} x_{i}} \iota_{i}\left(C_{i}\right)\right)^{\perp} \text { for all }\left(r_{i}, x_{i}\right) \in(0, \epsilon) \times L_{i}
$$

and

$$
\left|\nabla_{i}^{j}\left(\Phi_{i}\left(r_{i}, x_{i}\right)-\iota_{i}\left(r_{i}, x_{i}\right)\right)\right|=O\left(r_{i}^{\mu-j}\right) \quad \text { for } j \in \mathbb{N} \text { as } r_{i} \rightarrow 0
$$

where $\iota_{i}\left(r_{i}, x_{i}\right)=r_{i} x_{i} \in B\left(0 ; \epsilon_{M}\right), \nabla_{i}$ is the Levi-Civita connection of the cone metric $g_{i}=d r_{i}^{2}+r_{i}^{2} h_{i}$ on $C_{i}=(0, \infty) \times L_{i}$ coupled with partial differentiation on $\mathbb{R}^{7}$, and $|$.$| is calculated with respect to g_{i}$.

We call $C_{i}$ the cone at the singularity $z_{i}$ and $L_{i}$ the link of the cone $C_{i}$. We may write $N$ as the disjoint union $N=K_{N} \sqcup \bigsqcup_{i=1}^{s} U_{i}$, where $K_{N}$ is compact.

If $\hat{N}$ is coassociative in $M$, we say that $N$ is a $C S$ coassociative 4 -fold.

Remark. If $N$ is a CS 4 -fold, $\hat{N}$ is non-compact. 
Suppose $N$ is a CS 4 -fold at $z_{1}, \ldots, z_{s}$ with rate $\mu$ in $\left(M, \varphi, g_{\varphi}\right)$ and use the notation of Definition 3.2. The induced metric on $\hat{N},\left.g_{\varphi}\right|_{\hat{N}}$, makes $\hat{N}$ into a Riemannian manifold. Moreover, it is clear from (3.2) that, as long as $\mu<2$, the maps $\Psi_{i}$ satisfy

$$
\left|\nabla_{i}^{j}\left(\Psi_{i}^{*}\left(\left.g_{\varphi}\right|_{\hat{N}}\right)-g_{i}\right)\right|=O\left(r_{i}^{\mu-1-j}\right) \quad \text { for } j \in \mathbb{N} \text { as } r_{i} \rightarrow 0 \text {. }
$$

Consequently, the condition $\mu>1$ guarantees that the induced metric on $\hat{N}$ genuinely converges to the conical metric on $C_{i}$.

Note. As shown on [25, p. 6], since $\mu \in(1,2)$, Definition 3.2 is independent of the choice of $\mathrm{G}_{2}$ coordinate system near the singularities, up to equivalence.

Definition 3.3. Let $N$ be a CS coassociative 4-fold in an almost $\mathrm{G}_{2}$ manifold and use the notation of Definition 3.2. A radius function on $\hat{N}$ is a smooth map $\rho: \hat{N} \rightarrow(0,1]$ such that there exist positive constants $c_{1}<1$ and $c_{2}>1$ with $c_{1} r_{i}<\Psi_{i}^{*}(\rho)<c_{2} r_{i}$ on $(0, \epsilon) \times L_{i}$ for $i=1, \ldots, s$.

It is clear how we may construct such a function.

We now make a definition that also depends only on equivalence classes of $\mathrm{G}_{2}$ coordinate systems near the singularities.

Definition 3.4. Let $N$ be a CS coassociative 4 -fold at $z_{1}, \ldots, z_{s}$ in an almost $\mathrm{G}_{2}$ manifold. Use the notation of Definitions 3.1 and 3.2. For $i=$ $1, \ldots, s$, define a cone $\hat{C}_{i}$ in $T_{z_{i}} M$ by $\hat{C}_{i}=\left(\zeta_{i} \circ \iota_{i}\right)\left(C_{i}\right)$. We call $\hat{C}_{i}$ the tangent cone at $z_{i}$.

Using (3.2), one sees that $\hat{C}_{i}$ is a tangent cone at $z_{i}$ in the sense of Definition 2.6. Since the tangent cone has multiplicity one, [37, Theorem 5.7] implies that $\hat{C}_{i}$ is the unique tangent cone to $N$ at $z_{i}$. It is still an open question whether a general calibrated integral current has a unique tangent cone at each point.

We conclude with a straightforward result that follows from Proposition 2.7 or, by more elementary means, from [25, Proposition 3.6].

Proposition 3.5. Let $N$ be a CS coassociative 4 -fold at $z_{1}, \ldots, z_{s}$ in an almost $\mathrm{G}_{2}$ manifold. The tangent cones at $z_{1}, \ldots, z_{s}$ are coassociative. 


\subsection{Weighted Banach spaces}

For this subsection we let $(P, g)$ denote the non-singular part of a CS coassociative 4-fold in an almost $\mathrm{G}_{2}$ manifold with the induced metric and let $\rho$ be a radius function on $P$, as given in Definitions 3.2 and 3.3. We also let $\nabla$ denote the Levi-Civita connection of $g$. We define weighted Banach spaces of forms on $P$ as in $[2$, Section 1] so as to implement the analytic framework of $[25]$.

We shall use the notation and definition of the usual "unweighted" Banach spaces of forms as in [13, Section 1.2]; that is, Sobolev and Hölder spaces are denoted by $L_{k}^{p}$ and $C^{k, a}$, respectively, where $p \geq 1, k \in \mathbb{N}$ and $a \in(0,1)$. Recall that, by the Sobolev Embedding Theorem, $L_{k}^{p}$ embeds continuously in $L_{l}^{q}$ if $l \leq k$ and $l-\frac{4}{q} \leq k-\frac{4}{p}$, and $L_{k}^{p}$ embeds continuously in $C^{l, a}$ if $k-\frac{4}{p} \geq l+a$. We also introduce the notation $C_{\text {loc }}^{k}$ for the space of forms $\xi$ such that $f \xi$ lies in $C^{k}$ for every smooth compactly supported function $f$, and similarly define spaces $L_{k, \text { loc }}^{p}$ and $C_{\mathrm{loc}}^{k, a}$.

Definition 3.6. Let $p \geq 1, k \in \mathbb{N}$ and $\lambda \in \mathbb{R}$. The weighted Sobolev space $L_{k, \lambda}^{p}\left(\Lambda^{m} T^{*} P\right)$ of $m$-forms $\xi$ on $P$ is the subspace of $L_{k, \text { loc }}^{p}\left(\Lambda^{m} T^{*} P\right)$ such that the norm

$$
\|\xi\|_{L_{k, \lambda}^{p}}=\sum_{j=0}^{k}\left(\int_{P}\left|\rho^{j-\lambda} \nabla^{j} \xi\right|^{p} \rho^{-4} d V_{g}\right)^{\frac{1}{p}}
$$

is finite. Then $L_{k, \lambda}^{p}\left(\Lambda^{m} T^{*} P\right)$ is a Banach space.

We now define the dual weighted Sobolev space that shall be useful later.

Definition 3.7. Use the notation from Definition 3.6. Let $p, q>1$ be such that $\frac{1}{p}+\frac{1}{q}=1$, let $k, l \in \mathbb{N}$ and let $\lambda \in \mathbb{R}$. We then define $\langle.,$.$\rangle :$ $L_{k, \lambda}^{p}\left(\Lambda^{m} T^{*} P\right) \times L_{l,-4-\lambda}^{q}\left(\Lambda^{m} T^{*} P\right) \rightarrow \mathbb{R}$ by

$$
\langle\xi, \eta\rangle=\int_{P} \xi \wedge * \eta
$$

We shall refer to this as the dual pairing. For our purposes, we take the dual space of $L_{k, \lambda}^{p}\left(\Lambda^{m} T^{*} P\right)$ to be $L_{l,-4-\lambda}^{q}\left(\Lambda^{m} T^{*} P\right)$, with linear functionals represented by dual pairings. 
Definition 3.8. Let $\lambda \in \mathbb{R}$ and $k \in \mathbb{N}$. The weighted $C^{k}$-space $C_{\lambda}^{k}\left(\Lambda^{m} T^{*} P\right)$ of $m$-forms $\xi$ on $P$ is the subspace of $C_{\mathrm{loc}}^{k}\left(\Lambda^{m} T^{*} P\right)$ such that the norm

$$
\|\xi\|_{C_{\lambda}^{k}}=\sum_{j=0}^{k} \sup _{P}\left|\rho^{j-\lambda} \nabla^{j} \xi\right|
$$

is finite. We define $C_{\lambda}^{\infty}\left(\Lambda^{m} T^{*} P\right)=\bigcap_{k \geq 0} C_{\lambda}^{k}\left(\Lambda^{m} T^{*} P\right)$. Then $C_{\lambda}^{k}\left(\Lambda^{m} T^{*} P\right)$ is a Banach space, but $C_{\lambda}^{\infty}\left(\Lambda^{m} T^{*} P\right)$ is not in general.

Definition 3.9. Let $E$ be a vector bundle on $P$ endowed with Euclidean metrics on its fibres and a connection preserving these metrics. Let $d(x, y)$ be the geodesic distance between points $x, y \in P$, let $a \in(0,1)$, let $k \in \mathbb{N}$ and let $\lambda \in \mathbb{R}$. Let

$$
\begin{aligned}
& H=\left\{(x, y) \in P \times P: x \neq y, c_{1} \rho(x) \leq \rho(y) \leq c_{2} \rho(x)\right. \text { and } \\
&\text { there exists a geodesic in } P \text { of length } d(x, y) \text { from } x \text { to } y\},
\end{aligned}
$$

where $0<c_{1}<1<c_{2}$ are constant. A section $s$ of $E$ is Hölder continuous (with exponent $a$ ) if

$$
[s]^{a}=\sup _{(x, y) \in H} \frac{|s(x)-s(y)|_{E}}{d(x, y)^{a}}<\infty .
$$

We understand the quantity $|s(x)-s(y)|_{E}$ as follows. Given $(x, y) \in H$, there exists a geodesic $\gamma$ of length $d(x, y)$ connecting $x$ and $y$. Parallel translation along $\gamma$ using the connection on $E$ identifies the fibres over $x$ and $y$ and the metrics on them. Thus, with this identification, $|s(x)-s(y)|_{E}$ is well-defined.

The weighted Hölder space $C_{\lambda}^{k, a}\left(\Lambda^{m} T^{*} P\right)$ is defined to be the subspace of $C_{\mathrm{loc}}^{k, a}\left(\Lambda^{m} T^{*} P\right)$ such that the norm

$$
\|\xi\|_{C_{\lambda}^{k, a}}=\|\xi\|_{C_{\lambda}^{k}}+[\xi]_{\lambda}^{k, a}
$$

is finite, where

$$
[\xi]_{\lambda}^{k, a}=\left[\rho^{k+a-\lambda} \nabla^{k} \xi\right]^{a} .
$$

Then $C_{\lambda}^{k, a}\left(\Lambda^{m} T^{*} P\right)$ is a Banach space. It is clear that we have embeddings $C_{\lambda}^{k, a}\left(\Lambda^{m} T^{*} P\right) \hookrightarrow C_{\lambda}^{l}\left(\Lambda^{m} T^{*} P\right)$ and $C_{\lambda}^{k+1}\left(\Lambda^{m} T^{*} P\right) \hookrightarrow C_{\lambda}^{l, a}\left(\Lambda^{m} T^{*} P\right)$ if $l \leq k$.

Remark. The set $H$ in Definition 3.9 is introduced so that $[\xi]_{\lambda}^{k, a}$ is well-defined. 
Finally, we shall need the analogue of the Sobolev Embedding Theorem for weighted spaces, which is adapted from [23, Lemma 7.2] and [2, Theorem 1.2].

Theorem 3.10 (Weighted Sobolev Embedding Theorem). Let $p, q \geq 1, a \in(0,1), \lambda, \nu \in \mathbb{R}$ and $k, l \in \mathbb{N}$.

(a) If $k \geq l, k-\frac{4}{p} \geq l-\frac{4}{q}$, and either $p \leq q$ and $\lambda \geq \nu$, or $p>q$ and $\lambda>$ $\nu$, there is a continuous embedding $L_{k, \lambda}^{p}\left(\Lambda^{m} T^{*} P\right) \hookrightarrow L_{l, \nu}^{q}\left(\Lambda^{m} T^{*} P\right)$.

(b) If $k-\frac{4}{p} \geq l+a$, there is a continuous embedding $L_{k, \lambda}^{p}\left(\Lambda^{m} T^{*} P\right) \hookrightarrow$ $C_{\lambda}^{l, a}\left(\Lambda^{m} T^{*} P\right)$.

\subsection{Deformation theory}

We now review and discuss the key deformation theory results for CS coassociative 4-folds from [25]. We begin by recalling the linear differential operator governing infinitesimal deformations of CS coassociative 4-folds.

Definition 3.11. Let $N$ be a CS coassociative 4-fold in an almost $\mathrm{G}_{2}$ manifold. Let $p>4, k \geq 2$ and let $\lambda \in \mathbb{R}$. Define

$$
\left(\mathrm{d}_{+}+\mathrm{d}^{*}\right)_{\lambda}: L_{k+1, \lambda}^{p}\left(\Lambda_{+}^{2} T^{*} \hat{N} \oplus \Lambda^{4} T^{*} \hat{N}\right) \rightarrow L_{k, \lambda-1}^{p}\left(\Lambda^{3} T^{*} \hat{N}\right)
$$

by $\left(\mathrm{d}_{+}+\mathrm{d}^{*}\right)_{\lambda}(\alpha, \beta)=\mathrm{d} \alpha+\mathrm{d}^{*} \beta$.

Notes. We use the operator (3.4) rather than simply the exterior derivative on self-dual 2 -forms since the former operator is elliptic, whereas the latter is not. The choice of $p>4$ and $k \geq 2$ ensures that $L_{k+1, \lambda}^{p}$-solutions to the (non-linear) deformation problem are in fact smooth.

Definition 3.12. Let $N$ be a CS coassociative 4-fold in an almost $\mathrm{G}_{2}$ manifold and use the notation of Definition 3.2. Let

$$
\begin{gathered}
D(\lambda, i)=\left\{\left(\alpha_{i}, \beta_{i}\right) \in C^{\infty}\left(\Lambda^{2} T^{*} L_{i} \oplus \Lambda^{3} T^{*} L_{i}\right): \mathrm{d}_{i} \alpha_{i}=-\lambda \beta_{i},\right. \\
\left.\mathrm{d}_{i} *_{i} \alpha_{i}+\mathrm{d}_{i}^{*} \beta_{i}=-(\lambda+2) \alpha_{i}\right\},
\end{gathered}
$$

where $*_{i}, \mathrm{~d}_{i}$ and $\mathrm{d}_{i}^{*}$ are the Hodge star, the exterior derivative and its formal adjoint on $L_{i}$. By [25, Propositions 5.1 and 5.2], the set $\mathcal{D}$ of real numbers 
such that (3.4) is not Fredholm is countable and discrete and given by

$$
\mathcal{D}=\bigcup_{i=1}^{s}\{\lambda: D(\lambda, i) \neq 0\} .
$$

We also set $d(\lambda)=\sum_{i=1}^{s} \operatorname{dim} D(\lambda, i)$.

Note. The forms $\left(\alpha_{i}, \beta_{i}\right) \in D(\lambda, i)$ correspond to homogeneous forms $(\alpha, \beta) \in C^{\infty}\left(\Lambda_{+}^{2} T^{*} C_{i} \oplus \Lambda^{4} T^{*} C_{i}\right)$ of order $\lambda$ which satisfy $\mathrm{d} \alpha+\mathrm{d}^{*} \beta=0$ on the cone $C_{i}$.

In [25] the author studied deformations of CS coassociative 4-folds, allowing the singularities and tangent cones to vary, and permitting changes in the ambient $\mathrm{G}_{2}$ structure. However, for our purposes, we require a slightly more general theory, where we allow the underlying cones on which the singularities are modelled to vary as well. We now define the moduli space of deformations.

Definition 3.13. Let $N$ be a CS coassociative 4-fold in an almost $\mathrm{G}_{2}$ manifold $\left(M, \varphi, g_{\varphi}\right)$ and let $\mathcal{D}$ be given by Definition 3.12. Suppose further that $N$ has singularities at $z_{1}, \ldots, z_{s}$ with rate $\mu \in(1,2) \backslash \mathcal{D}$, having cone $C_{i}$ and tangent cone $\hat{C}_{i}$ at $z_{i}$ for all $i$. Let $\mathfrak{C}=\prod_{i=1}^{s} \mathcal{C}_{i}$ where $\mathcal{C}_{i}$ is a smooth, connected family of coassociative cones in $\mathbb{R}^{7}$, closed under the natural action of $\mathrm{G}_{2} \ltimes \mathbb{R}^{7}$, such that $C_{i} \in \mathcal{C}_{i}$ for all $i$.

The moduli space of deformations $\mathcal{M}(N, \mu, \mathfrak{C})$ is the set of CS coassociative 4-folds $N^{\prime}$ in $M$ such that:

(a) $N^{\prime}$ has a singularity at $z_{i}^{\prime}$ with rate $\mu$ and cone in $\mathcal{C}_{i}$ for $i=1, \ldots, s$; and

(b) there exists a diffeomorphism $h: M \rightarrow M$, isotopic to the identity, such that $h\left(z_{i}\right)=z_{i}^{\prime}$ for all $i,\left.h\right|_{N}: N \rightarrow N^{\prime}$ is a homeomorphism and $\left.h\right|_{\hat{N}}: \hat{N} \rightarrow N^{\prime} \backslash\left\{z_{1}^{\prime}, \ldots, z_{s}^{\prime}\right\}$ is a diffeomorphism.

We state the deformation theory result we require.

Theorem 3.14. Use the notation of Definition 3.13, let $p>4$ and $k \geq 2$. There exist finite-dimensional vector spaces $\mathcal{I}(N, \mu, \mathfrak{C})$ and $\mathcal{O}(N, \mu, \mathfrak{C})$, with $\mathcal{O}(N, \mu, \mathfrak{C})$ contained in $L_{k, \mu-1}^{p}\left(\Lambda^{3} T^{*} \hat{N}\right)$, and there exist

(a) a smooth manifold $\hat{\mathcal{M}}(N, \mu, \mathfrak{C})$, which is an open neighbourhood of 0 in $\mathcal{I}(N, \mu, \mathfrak{C})$, and 
(b) a smooth map $\pi: \hat{\mathcal{M}}(N, \mu, \mathfrak{C}) \rightarrow \mathcal{O}(N, \mu, \mathfrak{C})$, with $\pi(0)=0$,

such that an open neighbourhood of zero in Ker $\pi$ is homeomorphic to an open neighbourhood of $N$ in $\mathcal{M}(N, \mu, \mathfrak{C})$.

Furthermore, if $\mathcal{O}(N, \mu, \mathfrak{C})=\{0\}$, then $\mathcal{M}(N, \mu, \mathfrak{C})$ is a smooth manifold of dimension equal to that of $\mathcal{I}(N, \mu, \mathfrak{C})$.

We can actually say more about the spaces $\mathcal{I}(N, \mu, \mathfrak{C})$ and $\mathcal{O}(N, \mu, \mathfrak{C})$.

Proposition 3.15. Use the notation of Definitions 3.11-3.13 and Theorem 3.14.

(a) $\mathcal{I}(N, \mu, \mathfrak{C})$ contains a subspace isomorphic to $\operatorname{Ker}\left(\mathrm{d}_{+}+\mathrm{d}^{*}\right)_{\mu}$.

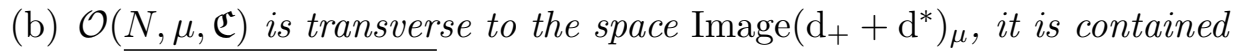
in $\overline{\mathrm{d}\left(L_{k+1, \mu}^{p}\left(\Lambda^{2} T^{*} \hat{N}\right)\right)} \subseteq L_{k, \mu-1}^{p}\left(\Lambda^{3} T^{*} \hat{N}\right)$ and satisfies

$$
\operatorname{dim} \mathcal{O}(N, \mu, \mathfrak{C}) \leq \sum_{\lambda \in(-2, \mu) \cap \mathcal{D}} d(\lambda)-\sum_{i=1}^{s} \operatorname{dim} \mathcal{C}_{i}
$$

These results essentially follow from [25, Theorem 7.9 and Proposition 8.10], the only difference being that we allow the cones $C_{i}$ on which the singularities are modelled to deform in the families $\mathcal{C}_{i}$, which may be larger than the families given simply by translations and $\mathrm{G}_{2}$ transformations of $C_{i}$. Rather than repeating the entire analysis in [25] with this change, we appeal to the similar discussion in [16, Section 8.3]; that is, the infinitesimal deformation space $\mathcal{I}(N, \mu, \mathfrak{C})$ is unchanged but the dimension of the obstruction space $\mathcal{O}(N, \mu, \mathfrak{C})$ is reduced by the dimension of the families in which the cones vary.

Notes. In $(3.5)$, the sum is over $(-2, \mu) \cap \mathcal{D}$, rather than $(-1, \mu) \cap \mathcal{D}$ as in $[25$, Proposition 8.10]. This ability to improve to the latter smaller set was based on the claim in [25, Proposition 5.3], which followed from applying $[23$, Theorem 10.2], that $(-2,-1] \cap \mathcal{D}=\emptyset$. Although $(-2,-1) \cap \mathcal{D}=\emptyset$ for almost all examples of interest, often $-1 \in \mathcal{D}$ so the claim is erroneous. However, we shall show in Proposition 4.11 that not only is [25, Proposition 8.10] valid, but the estimate in (3.5) can be improved even further.

In [25, Theorem 7.13] we proved a result for deformations of $N$ where the ambient $\mathrm{G}_{2}$ structure on $M$ also varies. We see that we have to restrict our choice of perturbations of the $\mathrm{G}_{2}$ structure as follows. 
Definition 3.16. Use the notation of Definition 3.13. By [25, Proposition 6.19] there exists a neighbourhood $T_{N}$ of $N$ in $M$, which retracts onto $N$ and $H_{\mathrm{dR}}^{3}\left(T_{N}\right) \cong H_{\mathrm{cs}}^{3}(\hat{N})$, the third compactly supported cohomology group of $\hat{N}$. Thus, any closed positive 3 -form $\varphi^{\prime}$ on $M$ defines a cohomology class $\left[\left.\varphi^{\prime}\right|_{\hat{N}}\right] \in H_{\mathrm{cs}}^{3}(\hat{N})$ and, moreover, if $\left[\left.\varphi^{\prime}\right|_{\hat{N}}\right] \neq 0$ in $H_{\mathrm{cs}}^{3}(\hat{N})$ there are $n o$ nearby coassociative deformations of $N$. Therefore, we let $\mathcal{F}=\left\{\left(\varphi^{f}, g_{\varphi^{f}}\right)\right.$ : $\left.f \in B(0 ; \delta) \subseteq \mathbb{R}^{r}\right\}$ be a smooth $r$-dimensional family of closed $\mathrm{G}_{2}$ structures on $M$, with $\varphi^{0}=\varphi$ and $\left[\left.\varphi^{f}\right|_{\hat{N}}\right]=0$ for all $f \in B(0 ; \delta)$.

The obstruction space for the deformation problem where the ambient $\mathrm{G}_{2}$ structure deforms in the family $\mathcal{F}$, suitably generalized to include the possibility that the cones at the singularities vary in the family $\mathfrak{C}$, is contained in $\mathcal{O}(N, \mu, \mathfrak{C})$. Therefore, if $\mathcal{O}(N, \mu, \mathfrak{C})=\{0\}$, for any sufficiently small perturbation of the $\mathrm{G}_{2}$ structure $\left(\varphi, g_{\varphi}\right)$ in $\mathcal{F}$ we obtain a corresponding CS deformation of $N$ which is coassociative with respect to the new $\mathrm{G}_{2}$ structure. Thus, we have the following result using [25, Theorem 7.13].

Theorem 3.17. Use the notation of Definitions 3.13 and 3.16 and Theorem 3.14. If $\mathcal{O}(N, \mu, \mathfrak{C})=\{0\}$, there exists $\delta_{N} \in(0, \delta)$ such that, if $f \in$ $B\left(0 ; \delta_{N}\right)$, then there exists a CS deformation $N^{f}$ of $N$ in $T_{N}$ with s singularities with rate $\mu$, modelled on cones in $\mathfrak{C}$, which is coassociative with respect to $\varphi^{f}$.

Note. By the method of proof of [25, Theorem 7.13], a "sufficiently small" perturbation $\left(\varphi^{\prime}, g_{\varphi^{\prime}}\right)$ of $\left(\varphi, g_{\varphi}\right)$ is one for which $\left\|\varphi^{\prime}-\varphi\right\|_{C^{1}}<\epsilon_{C^{1}}$ and $\| \varphi^{\prime}-$ $\varphi \|_{L_{2}^{p}}<\epsilon_{L_{2}^{p}}$ for some $p>4$, where the norms are calculated in $T_{N}$ with respect to $g_{\varphi}$, and $\epsilon_{C^{1}}$ and $\epsilon_{L_{2}^{p}}$ are small constants determined by the geometry of $\left(M, \varphi, g_{\varphi}\right)$ near $N$ (i.e., in $\left.T_{N}\right)$. Thus $\delta_{N}$ is chosen so that these $C^{1}$ and $L_{2}^{p}$ norms are smaller than the appropriate constants.

\subsection{Multiplicity one tangent cones}

In this subsection, we study coassociative integral currents with multiplicity one tangent cones, motivated by the work on special Lagrangian integral currents in [15, Section 6]. The key condition is that the underlying cone is Jacobi integrable, which we define for a coassociative cone following [15, Definition 6.7].

Definition 3.18. Let $C$ be a coassociative cone in $\mathbb{R}^{7}$ with compact link $L$ in $\mathcal{S}^{6}$ such that $C \backslash\{0\}$ is non-singular. We say that $v \in C^{\infty}\left(\nu_{\mathcal{S}^{6}}(L)\right)$ 
is a Lagrangian Jacobi field if $\left.\alpha_{v}=*(v\lrcorner \varphi_{0}\right)\left.\right|_{T L} \in C^{\infty}\left(T^{*} L\right)$ satisfies $\mathrm{d} \alpha_{v}=$ $-3 * \alpha_{v}$, where $*$ is the Hodge star on $L$. Notice that $v$ is a Lagrangian Jacobi field if and only if $r^{3} * \alpha_{v}+r^{2} \alpha_{v} \wedge \mathrm{d} r$ is a closed self-dual 2-form on $C$ of order $O(r)$, and so defines an infinitesimal deformation of $C$ as a coassociative cone; i.e., an infinitesimal deformation of $L$ as a Lagrangian in $\mathcal{S}^{6}$ in the sense of Definition 2.12. Since Lagrangians in $\mathcal{S}^{6}$ are minimal, Lagrangian Jacobi fields are Jacobi fields in the usual sense.

We say that $C$ is Jacobi integrable if every Lagrangian Jacobi field $v$ on $L$ defines a smooth one-parameter family $\left\{L_{t}=\exp _{t v}(L) \subseteq \mathcal{S}^{6}: t \in(-\tau, \tau)\right\}$, for some $\tau>0$, of Lagrangian submanifolds of $\mathcal{S}^{6}$.

Our next result proves Theorem 1.1; namely, that interior singular points of coassociative integral currents, with multiplicity one tangent cones modelled on Jacobi integrable cones, are conical singularities in the sense of Definition 3.2.

Theorem 3.19. Let $N$ be a coassociative integral current in an almost $\mathrm{G}_{2}$ manifold $M$ and let $z \in N^{\circ}$ be a singular point of $N$. Let $\left\{\chi: B\left(0 ; \epsilon_{M}\right) \rightarrow\right.$ $V\}$ be a $\mathrm{G}_{2}$ coordinate system near $z$ in the sense of Definition 3.1, with $\zeta=\left.\mathrm{d} \chi\right|_{0}$. Suppose that $C$ is a cone in $\mathbb{R}^{7}$ with compact link $L$ such that $C \backslash\{0\}$ is non-singular and $\hat{C}=\zeta(C) \subseteq T_{z} M$ is a multiplicity one tangent cone for $N$ at $z$. Then $C$ is coassociative and $\hat{C}$ is the unique tangent cone for $N$ at $z$.

Suppose further that $C$ is Jacobi integrable in the sense of Definition 3.18 and let $U=N \cap V$. Then there exist $\epsilon \in\left(0, \epsilon_{M}\right)$ and an embedding $\Phi:(0, \epsilon) \times$ $L \rightarrow B\left(0 ; \epsilon_{M}\right)$ such that $U \backslash\{z\}=\chi \circ \Phi((0, \epsilon) \times L)$ as an embedded submanifold of $M$ and $\Phi$ satisfies (3.1) and (3.2) for some $\mu \in(1,2)$.

Proof. The fact that $C$ is coassociative follows from Proposition 2.7. By [37, Theorem 5.7], the tangent cone $\hat{C}$ is unique and $U \backslash\{z\}$ can be realized as a $C^{2}$-embedding $\Psi$ of $(0, \epsilon) \times L$, for some $\epsilon>0$. Moreover, $\Psi=\chi \circ \Phi$ where $\Phi$ is a $C^{2}$-embedding of $(0, \epsilon) \times L$ into $B\left(0 ; \epsilon_{M}\right)$ which satisfies (3.1). This last point is not explicit in the statement of the theorem, but does follow from the proof. Moreover, if $\iota: C \backslash\{0\} \cong(0, \infty) \times L \rightarrow \mathbb{R}^{7}$ is the inclusion map, $\Phi$ also satisfies:

$$
\begin{aligned}
|\Phi(r, x)-\iota(r, x)| & =o(r), \\
|\nabla(\Phi(r, x)-\iota(r, x))| & =o(1), \\
\left|\nabla^{2}(\Phi(r, x)-\iota(r, x))\right| & =O(1)
\end{aligned}
$$


as $r \rightarrow 0$. Equation (3.8) again is not stated explicitly in [37, Theorem 5.7] but follows from the minimality of $C$ and $N$ as discussed in the proof of [1, Theorem 1]. The aforementioned theorem [1, Theorem 1] and its proof imply that there exists $\mu \in(1,2)$ such that the estimates (3.6) and (3.7) can be improved to:

$$
\begin{aligned}
|\Phi(r, x)-\iota(r, x)| & =O\left(r^{\mu}\right), \\
|\nabla(\Phi(r, x)-\iota(r, x))| & =O\left(r^{\mu-1}\right)
\end{aligned}
$$

as $r \rightarrow 0$, since $C$ is Jacobi integrable. We now only need a regularity argument to complete the proof, similar to [27, Proposition 4.17], which we now detail.

Let $P=\iota((0, \epsilon) \times L) \subseteq \mathbb{R}^{7}$ and recall that, since $P$ is coassociative, we have an isomorphism $\jmath_{P}: \nu(P) \rightarrow \Lambda_{+}^{2} T^{*} P$ by Proposition 2.17. For $\alpha \in$ $C_{\mathrm{loc}}^{2}\left(\Lambda_{+}^{2} T^{*} P\right)$ and $v=\jmath_{P}^{-1}(\alpha)$, define

$$
F(\alpha)=\exp _{v}^{*}\left(\left.\varphi_{0}\right|_{\exp _{v}(P)}\right) \in C_{\mathrm{loc}}^{1}\left(\Lambda^{3} T^{*} P\right)
$$

and

$$
G(\alpha)=\pi_{\Lambda_{+}^{2}}\left(\mathrm{~d}^{*} F(\alpha)\right) \in C_{\mathrm{loc}}^{0}\left(\Lambda_{+}^{2} T^{*} P\right)
$$

where $\pi_{\Lambda_{+}^{2}}$ is the projection from 2-forms to self-dual 2-forms on $P$. Clearly, if $F(\alpha)=0$, the deformation $\exp _{v}(P)$ of $P$ is coassociative and $G(\alpha)=0$. Moreover, $\left.\mathrm{d} F\right|_{0}(\alpha)=\mathrm{d} \alpha$ by $\left[31\right.$, p. 731] and thus $\left.\mathrm{d} G\right|_{0}(\alpha)=\mathrm{d}_{+}^{*} \mathrm{~d} \alpha$, where $\mathrm{d}_{+}^{*}=\pi_{\Lambda_{+}^{2}} \circ \mathrm{d}^{*}$ acting on 3 -forms on $P$. We deduce that $G(\alpha)=0$ is a nonlinear elliptic equation for $\alpha$ at 0 ; that is, its linearization at 0 is elliptic.

Since $\Phi$ is a $C^{2}$-coassociative embedding satisfying (3.1), it defines a $C^{2}$ normal vector field $v_{\Phi}$ on $P$ and hence a $C^{2}$-self-dual 2-form $\alpha_{\Phi}=\jmath_{P}\left(v_{\Phi}\right)$ on $P$ which satisfies $F\left(\alpha_{\Phi}\right)=0$. Moreover, $\alpha_{\Phi} \in C_{\mu}^{2}\left(\Lambda_{+}^{2} T^{*} P\right)$ by $(3.9)-(3.10)$ and (3.8), since $\mu-2<0$. Thus, $\alpha_{\Phi} \in C_{\mu}^{1, a}\left(\Lambda_{+}^{2} T^{*} P\right)$ for any $a \in(0,1)$.

Let $\nabla$ be the Levi-Civita connection of the conical metric on $P$. We can write, for $\alpha \in C_{\mathrm{loc}}^{2}\left(\Lambda_{+}^{2} T^{*} P\right)$ and $p \in P$,

$$
G(\alpha)(p)=R(p, \alpha(p), \nabla \alpha(p)) \nabla^{2} \alpha(p)+E(p, \alpha(p), \nabla \alpha(p))
$$

where $R$ and $E$ are smooth functions of their arguments, since $G(\alpha)$ is linear in $\nabla^{2} \alpha$ and a smooth function of $\alpha$. This leads us to define a new operator 
on $\beta \in C_{\mathrm{loc}}^{2}\left(\Lambda_{+}^{2} T^{*} P\right)$ by

$$
S_{\Phi}(\beta)(p)=R\left(p, \alpha_{\Phi}(p), \nabla \alpha_{\Phi}(p)\right) \nabla^{2} \beta(p)
$$

Note that $S_{\Phi}$ is not the linearization of $G$ of 0 , but it is a linear second-order elliptic operator on $\beta$ with coefficients in $C_{\text {loc }}^{0, a}$.

Since $G\left(\alpha_{\Phi}\right)=0, S_{\Phi}\left(\alpha_{\Phi}\right)=-E\left(\alpha_{\Phi}\right)$, where we set

$$
E(\beta)(p)=E(p, \beta(p), \nabla \beta(p))
$$

for $p \in P$ and $\beta \in C_{\mathrm{loc}}^{2}\left(\Lambda_{+}^{2} T^{*} P\right)$. As argued in [27, Proposition 4.17], $E(\beta) \in$ $C_{\mu-1}^{k, a}$ if $\beta \in C_{\mu}^{k+1, a}$ since $E$ is no worse than quadratic in $\beta$ and $\nabla \beta$. Thus, using the Schauder regularity estimates as given in [30] and the fact that $\alpha_{\Phi} \in C_{\mu}^{1, a}$, we deduce that $\alpha_{\Phi} \in C_{\mu}^{2, a}\left(\Lambda_{+}^{2} T^{*} P\right)$. A standard inductive argument leads us to the conclusion that $\alpha_{\Phi} \in C_{\mu}^{k, a}$ for all $k \in \mathbb{N}$. Thus the corresponding map $\Phi$ satisfies (3.2) as claimed.

From Definition 3.2, we have an immediate corollary to Theorem 3.19.

Corollary 3.20. Let $N$ be a connected coassociative integral current in an almost $\mathrm{G}_{2}$ manifold with $\partial N=\emptyset$. If $N$ has multiplicity one tangent cones at its singular points modelled on Jacobi integrable coassociative cones, then $N$ is a CS coassociative 4-fold in the sense of Definition 3.2.

\section{Coassociative cones and stability index}

In this section, we define the notion of stability of conical singularities of coassociative 4-folds using a numerical invariant for a coassociative cone which we call the stability index. This stability index is calculated using the spectrum of the curl operator acting on 1-forms on the link of the cone. We begin by giving a brief survey of the known examples of coassociative cones.

\subsection{Examples}

For this subsection it is convenient to identify $\mathbb{R}^{7}$ with the imaginary octonions $\operatorname{Im} \mathbb{O}$. Let $\left\{\varepsilon_{1}, \ldots, \varepsilon_{7}\right\}$ be a basis for $\operatorname{Im} \mathbb{O}$ satisfying the multiplication 
law below.

\begin{tabular}{l|rrrrrrr} 
& $\varepsilon_{1}$ & $\varepsilon_{2}$ & $\varepsilon_{3}$ & $\varepsilon_{4}$ & $\varepsilon_{5}$ & $\varepsilon_{6}$ & $\varepsilon_{7}$ \\
\hline$\varepsilon_{1}$ & -1 & $\varepsilon_{3}$ & $-\varepsilon_{2}$ & $\varepsilon_{5}$ & $-\varepsilon_{4}$ & $\varepsilon_{7}$ & $-\varepsilon_{6}$ \\
$\varepsilon_{2}$ & $-\varepsilon_{3}$ & -1 & $\varepsilon_{1}$ & $\varepsilon_{6}$ & $-\varepsilon_{7}$ & $-\varepsilon_{4}$ & $\varepsilon_{5}$ \\
$\varepsilon_{3}$ & $\varepsilon_{2}$ & $-\varepsilon_{1}$ & -1 & $-\varepsilon_{7}$ & $-\varepsilon_{6}$ & $\varepsilon_{5}$ & $\varepsilon_{4}$ \\
$\varepsilon_{4}$ & $-\varepsilon_{5}$ & $-\varepsilon_{6}$ & $\varepsilon_{7}$ & -1 & $\varepsilon_{1}$ & $\varepsilon_{2}$ & $-\varepsilon_{3}$ \\
$\varepsilon_{5}$ & $\varepsilon_{4}$ & $\varepsilon_{7}$ & $\varepsilon_{6}$ & $-\varepsilon_{1}$ & -1 & $-\varepsilon_{3}$ & $-\varepsilon_{2}$ \\
$\varepsilon_{6}$ & $-\varepsilon_{7}$ & $\varepsilon_{4}$ & $-\varepsilon_{5}$ & $-\varepsilon_{2}$ & $\varepsilon_{3}$ & -1 & $\varepsilon_{1}$ \\
$\varepsilon_{7}$ & $\varepsilon_{6}$ & $-\varepsilon_{5}$ & $-\varepsilon_{4}$ & $\varepsilon_{3}$ & $\varepsilon_{2}$ & $-\varepsilon_{1}$ & -1
\end{tabular}

If we identify $\operatorname{Im} \mathbb{O} \cong \mathbb{R}^{7}$ so that $\left\{\varepsilon_{1}, \ldots, \varepsilon_{7}\right\}$ corresponds to the standard oriented orthonormal basis in $\mathbb{R}^{7}$, then the multiplication law we have chosen is consistent with the $\mathrm{G}_{2}$ structure on $\mathbb{R}^{7}$ given by $\varphi_{0}$ in $(2.1)$.

The coassociative cones invariant under a closed 3-dimensional Lie subgroup of $\mathrm{G}_{2}$ were classified in [29]. We now review these examples starting with the degenerate example of a coassociative cone, namely a coassociative 4-plane.

Example 4.1 (Planes). By [19, Proposition 12.1.2], $\mathrm{G}_{2}$ acts transitively on the set of coassociative 4-planes with isotropy $\mathrm{SO}(4)$. Therefore, any coassociative 4-plane is equivalent up to $\mathrm{G}_{2}$ transformation to

$$
C_{0}=\left\{x_{0} \varepsilon_{1}+x_{1} \varepsilon_{3}+x_{2} \varepsilon_{5}+x_{3} \varepsilon_{7} \in \operatorname{Im} \mathbb{O}:\left(x_{0}, x_{1}, x_{2}, x_{3}\right) \in \mathbb{R}^{4}\right\} .
$$

The link $L_{0}$ of $C_{0}$ is trivially a totally geodesic $\mathrm{SO}(4)$-invariant $\mathcal{S}^{3}$ in $\mathcal{S}^{6}$.

Our next example was first introduced in [22, Section 7] and shown to be coassociative in [10, Theorem IV.3.2].

Example 4.2 (U(2) symmetry). The cone $C_{1}$ in Im $\mathbb{O}$, with link

$$
L_{1}=\left\{\frac{\sqrt{5}}{3} \bar{q} \varepsilon_{1} q+\frac{2}{3} q \varepsilon_{5}: q \in\left\langle 1, \varepsilon_{1}, \varepsilon_{2}, \varepsilon_{3}\right\rangle_{\mathbb{R}} \text { with }|q|=1\right\}
$$

is coassociative and invariant under a $\mathrm{U}(2)$ subgroup of $\mathrm{G}_{2}$. The Lagrangian $L_{1}$ is realized as an $\operatorname{Sp}(1)$-orbit in $\mathcal{S}^{6}$.

Recall that, by Corollary 2.11, any complex 2-dimensional cone in $\mathbb{C}^{3}$ embedded in $\mathbb{R}^{7}$ is coassociative. 
Example 4.3 (Complex SO(3) symmetry). Identify $\mathbb{R}^{7} \cong \mathbb{R} \oplus \mathbb{C}^{3}$ as in Lemma 2.9. The cone

$$
C_{2}=\left\{\left(0, z_{1}, z_{2}, z_{3}\right) \in \mathbb{R} \oplus \mathbb{C}^{3}: z_{1}^{2}+z_{2}^{2}+z_{3}^{2}=0\right\}
$$

is coassociative and invariant under the standard $\mathrm{SO}(3)$ action on $\mathbb{C}^{3}$. The real link $L_{2}$ of $C_{2}$ in $\mathcal{S}^{6}$ is diffeomorphic to $\mathrm{SO}(3)$ and is the Hopf lift of the constant curvature degree $2 \mathbb{C P}^{1}$ in $\mathbb{C P}^{2}$.

Our final symmetric examples are most easily described using homogeneous harmonic cubics on $\mathbb{R}^{3}$.

Example 4.4 (SO(3) symmetry). Identify $\operatorname{Im} \mathbb{O}$ with the homogeneous harmonic cubics $\mathcal{H}^{3}\left(\mathbb{R}^{3}\right)$ on $\mathbb{R}^{3}$ by:

$$
\begin{array}{rlrl}
\varepsilon_{1} & \mapsto \frac{\sqrt{10}}{10} x\left(2 x^{2}-3 y^{2}-3 z^{2}\right) ; & & \\
\varepsilon_{2} \mapsto-\sqrt{6} x y z ; & \varepsilon_{3} \mapsto \frac{\sqrt{6}}{2} x\left(y^{2}-z^{2}\right) ; \\
\varepsilon_{4} \mapsto-\frac{\sqrt{15}}{10} y\left(4 x^{2}-y^{2}-z^{2}\right) ; & \varepsilon_{5} \mapsto-\frac{\sqrt{15}}{10} z\left(4 x^{2}-y^{2}-z^{2}\right) ; \\
\varepsilon_{6} \mapsto \frac{1}{2} y\left(y^{2}-3 z^{2}\right) ; & \varepsilon_{7} \mapsto-\frac{1}{2} z\left(z^{2}-3 y^{2}\right) .
\end{array}
$$

The standard $\mathrm{SO}(3)$ action on $\mathbb{R}^{3}$ induces an action on $\mathcal{H}^{3}\left(\mathbb{R}^{3}\right)$, hence on $\operatorname{Im}(\mathbb{O}$.

Let $L_{3}$ be the orbit through $\varepsilon_{6}$ of this $\mathrm{SO}(3)$ action on $\operatorname{Im} \mathbb{O}$ and let $L_{4}$ be the orbit through $\varepsilon_{2}$. By [29, Theorem 4.3] and observations in [28, Examples 6.6 and 6.15], $L_{3} \cong \mathrm{SO}(3) / \mathrm{S}_{3}$ and $L_{4} \cong \mathrm{SO}(3) / \mathrm{A}_{4}$ are Lagrangian. Thus the cones $C_{3}$ and $C_{4}$ on $L_{3}$ and $L_{4}$ respectively are coassociative and $\mathrm{SO}(3)$ invariant.

Note. The $\mathrm{SO}(3)$-orbit through $\epsilon_{1}$ is a constant curvature $\frac{1}{6}$ pseudoholomorphic curve in $\mathcal{S}^{6}$ often called the Boruoka sphere in $\mathcal{S}^{6}$.

All of the coassociative cones introduced so far have links which are fibred by oriented circles of constant radius over a surface. Lagrangians in $\mathcal{S}^{6}$ with this property were classified in [28]. This classification describes all of the known links of coassociative cones and it turns out that the circles have radius either $\frac{2}{3}$ or 1 . To describe these examples we make two definitions. 
Definition 4.5. Let $\mathbf{u}: \Sigma \rightarrow \mathcal{S}^{6}$ be a surface, let $\Pi$ be a 2-plane subbundle of $\mathbf{u}^{*}\left(T \mathcal{S}^{6}\right)$ and let $\mathcal{U}(\Pi)=\{\mathbf{v} \in \Pi:|\mathbf{v}|=1\}$. For $\gamma \in\left(0, \frac{\pi}{2}\right]$ we define $\mathbf{x}_{\gamma}$ : $\mathcal{U}(\Pi) \rightarrow \mathcal{S}^{6}$ by $\mathbf{x}_{\gamma}(\mathbf{v})=\cos \gamma \mathbf{u}+\sin \gamma \mathbf{v}$. We say that the image of $\mathbf{x}_{\gamma}$ is a tube of radius $\gamma$ (in $\Pi$ ) about $\Sigma$.

Definition 4.6. If $\mathbf{u}: \Sigma \rightarrow \mathcal{S}^{6}$ is a non-totally geodesic pseudoholomorphic curve in the sense of Definition 2.12, there is an orthogonal decomposition $\mathbf{u}^{*}\left(T^{1,0} \mathcal{S}^{6}\right)=T^{1,0} \Sigma \oplus N_{1} \Sigma \oplus N_{2} \Sigma$, where $N_{1} \Sigma$ and $N_{2} \Sigma$ are holomorphic line bundles such that the second fundamental form of $\mathbf{u}$ takes values in $N_{1} \Sigma$. We call $N_{1} \Sigma$ and $N_{2} \Sigma$ the first and second normal bundles, respectively.

If $\left(\mathbf{f}_{1}, \mathbf{f}_{2}, \mathbf{f}_{3}\right)$ is a moving orthonormal frame for $T^{1,0} \Sigma \oplus N_{1} \Sigma \oplus N_{2} \Sigma$ and $\theta_{1}$ is the $(1,0)$-form dual to $\mathbf{f}_{1}$, then the structure equations for $\Sigma$ are:

$$
\begin{aligned}
& \mathrm{d} \mathbf{u}=-2 i \mathbf{f}_{1} \theta_{1}+2 i \overline{\mathbf{f}}_{1} \bar{\theta}_{1} ; \quad \quad \mathrm{d} \mathbf{f}_{1}=-i \mathbf{u} \bar{\theta}_{1}+\mathbf{f}_{1} \kappa_{11}+\mathbf{f}_{2} \kappa_{21} ; \\
& \mathrm{d} \mathbf{f}_{2}=-\mathbf{f}_{1} \bar{\kappa}_{21}+\mathbf{f}_{2} \kappa_{22}+\mathbf{f}_{3} \kappa_{32}-\overline{\mathbf{f}}_{3} \theta_{1} ; \quad \mathrm{d} \mathbf{f}_{3}=-\mathbf{f}_{2} \bar{\kappa}_{32}+\mathbf{f}_{3} \kappa_{33}+\overline{\mathbf{f}}_{2} \theta_{1} ; \\
& \mathrm{d} \theta_{1}=-\kappa_{11} \wedge \theta_{1} ; \quad \mathrm{d} \kappa_{11}=-\kappa_{21} \wedge \bar{\kappa}_{21}+2 \theta_{1} \wedge \bar{\theta}_{1} ; \\
& \mathrm{d} \kappa_{22}=\kappa_{21} \wedge \bar{\kappa}_{21}-\kappa_{32} \wedge \bar{\kappa}_{32}-\theta_{1} \wedge \bar{\theta}_{1} ; \quad \mathrm{d} \kappa_{33}=\kappa_{32} \wedge \bar{\kappa}_{32}-\theta_{1} \wedge \bar{\theta}_{1} ; \\
& \mathrm{d} \kappa_{21}=\left(\kappa_{11}-\kappa_{22}\right) \wedge \kappa_{21} ; \quad \mathrm{d} \kappa_{32}=\left(\kappa_{22}-\kappa_{33}\right) \wedge \kappa_{32},
\end{aligned}
$$

for some imaginary-valued 1-forms $\kappa_{11}, \kappa_{22}, \kappa_{33}$ such that $\kappa_{11}+\kappa_{22}+\kappa_{33}=$ 0 and complex-valued 1 -forms $\kappa_{21}$ and $\kappa_{32}$. Moreover, by the work in [4, Section 4], there exist holomorphic functions $K$ and $T$ such that $\kappa_{21}=K \theta_{1}$ and $\kappa_{32}=T \theta_{1}$. We identify $K$ with the second fundamental form of $\Sigma$, so $K \neq 0$, and we call $T$ the torsion of $\Sigma$. Of particular interest are the pseudoholomorphic curves with null-torsion, i.e., with $T \equiv 0$, since they may be viewed as certain algebraic curves in the 5-quadric in $\mathbb{C P}^{6}$ and every compact Riemann surface can be realized as such a curve in $\mathcal{S}^{6}$ by the work in $[4$, Section 4].

Remark. The Borůvka sphere $\Sigma$ in $\mathcal{S}^{6}$ has null-torsion.

The coassociative cones with links which are fibred by circles of radius $\frac{2}{3}$ are locally cones over tubes of radius $\sin ^{-1}\left(\frac{2}{3}\right)$ in $N_{2} \Sigma$ about null-torsion pseudoholomorphic curves $\Sigma \subseteq \mathcal{S}^{6}$. This includes $C_{1}$ given in Example 4.2, where $\Sigma$ is a totally geodesic $\mathcal{S}^{2}$ (which is the degenerate case of a null-torsion curve), and $C_{4}$ given in Example 4.4, where $\Sigma$ is the Borůvka sphere.

We shall be more concerned with the case where the link is fibred by oriented geodesic circles so we make the following definition as in [24]. 
Definition 4.7. A 4-dimensional submanifold $N$ of $\mathbb{R}^{n}$ is 2-ruled if there is a surface $\Sigma$ and a smooth fibration $\pi: N \rightarrow \Sigma$ whose fibres are affine 2-planes in $\mathbb{R}^{n}$. In addition, we say that $N$ is $r$-framed if there is a choice of oriented frame for each 2-plane $\pi^{-1}(\sigma)$ which varies smoothly with $\sigma \in \Sigma$.

The 2-ruled coassociative 4-folds in $\mathbb{R}^{7}$ were studied in [24], and there was a further focus on the conical case in [9]. By [28, Theorems 1.1-1.2] we can extend the work in [9] and describe all r-framed 2-ruled coassociative cones. We refer the reader to [28] for full details but give a brief description here.

Example 4.8 (The 2-ruled family). Complex 2-dimensional cones in $\mathbb{C}^{3}$ embedded in $\mathbb{R}^{7}$ are 2-ruled coassociative cones by Corollary 2.11.

Recall Definition 4.5. The general r-framed 2-ruled coassociative cone $C$ has link $L$ such that, for all $p$ in an open dense subset $L^{*}$ of $L$, there exist an open set $U \ni p$, a non-totally geodesic pseudoholomorphic curve $\mathbf{u}: \Sigma \rightarrow \mathcal{S}^{6}$, and a holomorphic line subbundle $\Pi$ of $\mathbf{u}^{*}\left(T^{1,0} \mathcal{S}^{6}\right)$ such that $U \cap L^{*}$ is a tube of radius $\frac{\pi}{2}$ in $\Pi$ about $\Sigma$. There are restrictions on the choice of line subbundle $\Pi$ (see [28, Example 7.4]), however, in particular, we may always choose $\Pi=N_{2} \Sigma$.

We conclude this subsection with the following important examples of 2-ruled coassociative cones.

Example 4.9. Let $C$ be a coassociative cone with link $L$ admitting a Killing vector field whose integral curves are geodesic circles in $\mathcal{S}^{6}$. By [41, Theorem 2], either $C$ is a complex 2-dimensional cone in $\mathbb{C}^{3}$ or $L$ is locally a tube of radius $\frac{\pi}{2}$ in $N_{1} \Sigma$ or $N_{2} \Sigma$ about a null-torsion pseudoholomorphic curve $\Sigma$ in $\mathcal{S}^{6}$.

Cones as in Example 4.9 are given trivially by $C_{2}$ in Example 4.3 but also by $C_{3}$ in Example 4.4, since $L_{3}$ is a tube of radius $\frac{\pi}{2}$ in $N_{2} \Sigma$ about the Borůva sphere $\Sigma$.

\subsection{Stability index and the curl operator}

For this subsection, use the notation of Definitions 3.2 and 3.13 and Theorem 3.14. In particular, $N$ is a CS coassociative 4-fold where the $s$ singularities have rate $\mu \in(1,2) \backslash \mathcal{D}$, where $\mathcal{D}$ is given in Definition 3.12, and the $s$-tuple of cones at the singularities $\left(C_{1}, \ldots, C_{s}\right)$ lies in $\mathfrak{C}=\prod_{i=1}^{s} \mathcal{C}_{i}$. 
To get an effective notion of stability for the conical singularities of $N$ we need to improve the estimate (3.5) for the dimension of the obstruction space $\mathcal{O}(N, \mu, \mathfrak{C})$ for the deformation problem for $N$. To understand this we need to compare the maps $\left(\mathrm{d}_{+}+\mathrm{d}^{*}\right)_{\lambda}$ given by (3.4) and

$$
\begin{aligned}
\left(\mathrm{d}+\mathrm{d}^{*}\right)_{\lambda}: L_{k+1, \lambda}^{p}\left(\Lambda^{2} T^{*} \hat{N} \oplus \Lambda^{4} T^{*} \hat{N}\right) & \rightarrow L_{k, \lambda-1}^{p}\left(\Lambda^{3} T^{*} \hat{N}\right) \\
(\alpha, \beta) & \mapsto \mathrm{d} \alpha+\mathrm{d}^{*} \beta
\end{aligned}
$$

for $\lambda \in(-2, \mu]$. From Theorem 3.14, we see that $\mathcal{O}(N, \mu, \mathfrak{C})$ is a subspace of the cokernel of $\left(d_{+}+d^{*}\right)_{\mu}$ which is transverse to the cokernel of $\left(d+d^{*}\right)_{\mu}$. Moreover, from the work in [25, Section 8], the sum over $\lambda \in(-2, \mu) \cap \mathcal{D}$ in (3.5) is the upper bound of the dimension of the space of forms which adds to the cokernel of $\left(\mathrm{d}_{+}+\mathrm{d}^{*}\right)_{\lambda}$ as the rate $\lambda$ increases from -2 to $\mu$. Hence, we can improve the upper bound by considering the forms which add to the cokernel of $\left(\mathrm{d}_{+}+\mathrm{d}^{*}\right)_{\lambda}$ but not the cokernel of $\left(\mathrm{d}+\mathrm{d}^{*}\right)_{\lambda}$. These cokernels are isomorphic to the annihilators of the images of $\left(\mathrm{d}_{+}+\mathrm{d}^{*}\right)_{\lambda}$ and $\left(\mathrm{d}_{+} \mathrm{d}^{*}\right)_{\lambda}$ under the dual pairing given in Definition 3.7. Thus, by comparing these annihilators we may obtain our estimate.

We begin with the following.

Proposition 4.10. Let $\mathcal{A}_{+}(\lambda)$ and $\mathcal{A}(\lambda)$ denote the annihilators of the images of (3.4) and (4.1) via the dual pairing given in Definition 3.7. For $\lambda \leq-1, \mathcal{A}_{+}(\lambda)=\mathcal{A}(\lambda)$.

Proof. As observed in the proof of [25, Proposition 8.6], if $\lambda \notin \mathcal{D}$, there exist finite-dimensional spaces $\mathcal{C}_{+}(\lambda)$ and $\mathcal{C}(\lambda)$ of smooth compactly supported 3-forms on $\hat{N}$ such that

$$
L_{k, \lambda-1}^{p}\left(\Lambda^{3} T^{*} \hat{N}\right)=\operatorname{Image}\left(\mathrm{d}_{+}+\mathrm{d}^{*}\right)_{\lambda} \oplus \mathcal{C}_{+}(\lambda)=\overline{\operatorname{Image}\left(\mathrm{d}+\mathrm{d}^{*}\right)_{\lambda}} \oplus \mathcal{C}(\lambda)
$$

and the dual pairings between $\mathcal{C}_{+}(\lambda)$ and $\mathcal{A}_{+}(\lambda)$, and between $\mathcal{C}(\lambda)$ and $\mathcal{A}(\lambda)$, are non-degenerate. Let $\mathcal{C}^{\prime}(\lambda)$ be such that $\mathcal{C}_{+}(\lambda)=\mathcal{C}(\lambda) \oplus \mathcal{C}^{\prime}(\lambda)$.

Notice by Definition 3.7 that if $q>1$ is such that $\frac{1}{p}+\frac{1}{q}=1$ and $l \in \mathbb{N}$ then, for any $\lambda \in \mathbb{R}$,

$$
\begin{array}{r}
\mathcal{A}_{+}(\lambda)=\left\{\gamma \in L_{l+1,-3-\lambda}^{q}\left(\Lambda^{3} T^{*} \hat{N}\right):\left\langle\mathrm{d} \alpha+\mathrm{d}^{*} \beta, \gamma\right\rangle=0\right. \\
\text { for all } \left.(\alpha, \beta) \in L_{k+1, \lambda}^{p}\left(\Lambda_{+}^{2} T^{*} \hat{N} \oplus \Lambda^{4} T^{*} \hat{N}\right)\right\}
\end{array}
$$




$$
\begin{aligned}
= & \left\{\gamma \in L_{l+1,-3-\lambda}^{q}\left(\Lambda^{3} T^{*} \hat{N}\right):\left\langle\alpha, \mathrm{d}^{*} \gamma\right\rangle=0,\langle\beta, \mathrm{d} \gamma\rangle=0\right. \\
& \left.\quad \text { for all }(\alpha, \beta) \in L_{k+1, \lambda}^{p}\left(\Lambda_{+}^{2} T^{*} \hat{N} \oplus \Lambda^{4} T^{*} \hat{N}\right)\right\} \\
= & \left\{\gamma \in L_{l+1,-3-\lambda}^{q}\left(\Lambda^{3} T^{*} \hat{N}\right): \mathrm{d} \gamma=0, \mathrm{~d}^{*} \gamma \in L_{l,-4-\lambda}^{q}\left(\Lambda_{-}^{2} T^{*} \hat{N}\right)\right\}
\end{aligned}
$$

where the integration by parts is justified by the choice of weight for the dual weighted Sobolev space. Similarly,

$$
\mathcal{A}(\lambda)=\left\{\gamma \in L_{l+1,-3-\lambda}^{q}\left(\Lambda^{3} T^{*} \hat{N}\right): \mathrm{d} \gamma=\mathrm{d}^{*} \gamma=0\right\} .
$$

If $\lambda \leq-1$, then $-3-\lambda \geq \lambda-1$ so, by Theorem $3.10, L_{l+1,-3-\lambda}^{q} \hookrightarrow L_{k, \lambda-1}^{p}$ for sufficiently large $l$. Since $\mathcal{A}_{+}(\lambda)$ consists of smooth forms, as it is the kernel of an elliptic operator, we can choose $l$ arbitrarily large and see that $\mathcal{A}_{+}(\lambda) \subseteq L_{k, \lambda-1}^{p}\left(\Lambda^{3} T^{*} \hat{N}\right)$ and the same is clearly true for $\mathcal{A}(\lambda)$. Moreover, if additionally $\lambda \notin \mathcal{D}$, since $\mathcal{A}_{+}(\lambda)$ and $\mathcal{A}(\lambda)$ are of equal dimension to $\mathcal{C}_{+}(\lambda)$ and $\mathcal{C}(\lambda)$, and the annihilators are by construction orthogonal to the closures of the images of (3.4) and (4.1), we deduce that $\mathcal{A}_{+}(\lambda)=\mathcal{C}_{+}(\lambda)$ and $\mathcal{A}(\lambda)=\mathcal{C}(\lambda)$.

Suppose that $\gamma \in \mathcal{C}^{\prime}(\lambda)$. Then $\gamma$ is compactly supported and lies in $\mathcal{A}_{+}(\lambda)$. Therefore, since $\mathrm{d}^{*} \gamma$ is anti-self-dual,

$$
\left\|\mathrm{d}^{*} \gamma\right\|_{L^{2}}^{2}=\int_{\hat{N}}-\mathrm{d}^{*} \gamma \wedge \mathrm{d}^{*} \gamma=\int_{\hat{N}}-\mathrm{d} * \gamma \wedge \mathrm{d} * \gamma=\int_{\hat{N}}-\mathrm{d}(* \gamma \wedge \mathrm{d} * \gamma)=0
$$

where the integration by parts is valid since $\gamma$ is compactly supported. Thus, $\gamma \in \mathcal{A}(\lambda)=\mathcal{C}(\lambda)$, so $\gamma=0$. We deduce that $\mathcal{C}(\lambda)=\mathcal{C}_{+}(\lambda)=\mathcal{A}(\lambda)=$ $\mathcal{A}_{+}(\lambda)$ for $\lambda \leq-1, \lambda \notin \mathcal{D}$. The annihilators are well-defined for $\lambda \in \mathcal{D}$ and hence, since the dimension of $\mathcal{A}_{+}(\lambda)$ is lower semi-continuous at $\lambda=-1$, as remarked in the proof of $\left[25\right.$, Proposition 8.4], we see that $\mathcal{A}(\lambda)=\mathcal{A}_{+}(\lambda)$ for all $\lambda \leq-1$.

Proposition 4.11. Recall the notation of Definition 3.12. Let

$$
\check{D}(\lambda, i)=\left\{\gamma_{i} \in C^{\infty}\left(T^{*} L_{i}\right): \mathrm{d}_{i}^{*} \gamma_{i}=0, \mathrm{~d}_{i} \gamma_{i}=-(\lambda+2) *_{i} \gamma_{i}\right\}
$$

and let $\check{d}(\lambda)=\sum_{i=1}^{s} \operatorname{dim} \check{D}(\lambda, i)$. Then

$$
\operatorname{dim} \mathcal{O}(N, \mu, \mathfrak{C}) \leq \sum_{\lambda \in(-1, \mu) \cap \mathcal{D}} \check{d}(\lambda)-\sum_{i=1}^{s} \operatorname{dim} \mathcal{C}_{i} .
$$


Proof. By Proposition 4.10, there are no forms which add to the cokernel of (3.4) but not the cokernel of (4.1) for $\lambda \leq-1$. Therefore, we can first improve the estimate $(3.5)$ by restricting to the range of rates $(-1, \mu) \cap \mathcal{D}$.

The map $\left(d+d^{*}\right)_{\lambda}$, given in (4.1), is not elliptic, but forms part of the map

$$
\mathrm{d}+\mathrm{d}^{*}: L_{k+1, \lambda}^{p}\left(\Lambda^{\mathrm{even}} T^{*} \hat{N}\right) \rightarrow L_{k, \lambda-1}^{p}\left(\Lambda^{\text {odd }} T^{*} \hat{N}\right),
$$

which is elliptic. Therefore, we can apply the theory of [23] and deduce that there is a countable discrete set of rates $\mathcal{E} \supseteq \mathcal{D}$ for which $\mathrm{d}+\mathrm{d}^{*}$ is Fredholm and, moreover, we can calculate which forms on $L_{i}$ correspond to forms on $\hat{N}$ which subtract from the kernel or add to the cokernel as $\lambda$ increases.

The cokernel of $\mathrm{d}+\mathrm{d}^{*}$ is isomorphic to the annihilator of the image of $d+d^{*}$, using the dual pairing given in Definition 3.7. The work in [23] identifies the forms which add to this annihilator as the rate crosses elements $\lambda \in \mathcal{E}$. To calculate the changes in the annihilator, we need to consider homogeneous forms on the cone $C_{i}$ of the following type:

$$
\left(\gamma^{1}, \gamma^{3}\right)=\left(r^{-\lambda-2} \alpha^{1}+r^{-\lambda-3} \alpha^{0} \wedge \mathrm{d} r, r^{-\lambda} \alpha^{3}+r^{-\lambda-1} \alpha^{2} \wedge \mathrm{d} r\right)
$$

for $\left(\alpha^{0}, \alpha^{1}, \alpha^{2}, \alpha^{3}\right) \in C^{\infty}\left(\oplus_{m=0}^{3} \Lambda^{m} T^{*} L_{i}\right)$, which satisfy $\mathrm{d}^{*} \gamma^{1}=\mathrm{d} \gamma^{1}+\mathrm{d}^{*} \gamma^{3}=$ $\mathrm{d} \gamma^{3}=0$, where $\mathrm{d}^{*}$ is calculated using the cone metric. We therefore have the following conditions which define $\mathcal{E}$ and the changes to the kernel or cokernel of $\mathrm{d}+\mathrm{d}^{*}$ acting on even forms, using the notation of Definition 3.12:

$$
\begin{array}{ll}
\mathrm{d}_{i}^{*} \alpha^{1}=-\lambda \alpha^{0}, & \mathrm{~d}_{i} \alpha^{0}+\mathrm{d}_{i}^{*} \alpha^{2}=-(\lambda+2) \alpha^{1}, \\
\mathrm{~d}_{i} \alpha^{2}=-\lambda \alpha^{3}, & \mathrm{~d}_{i} \alpha^{1}+\mathrm{d}_{i}^{*} \alpha^{3}=-(\lambda+2) \alpha^{2} .
\end{array}
$$

Thus, by (4.3) and (4.4), forms $(\alpha, \beta) \in D(\lambda, i)$ giving rise to cokernel forms for $\left(\mathrm{d}_{+}+\mathrm{d}^{*}\right)_{\lambda}, \lambda \in \mathcal{D}$, which can lie in $\mathcal{O}(N, \mu, \mathfrak{C})$ must be transverse to forms $\left(\alpha^{\prime}, \beta^{\prime}\right) \in C^{\infty}\left(\Lambda^{2} T^{*} L_{i} \oplus \Lambda^{3} T^{*} L_{i}\right)$ satisfying:

$$
\mathrm{d}_{i} \alpha^{\prime}=-\lambda \beta^{\prime}, \quad \mathrm{d}_{i}^{*} \beta^{\prime}=-(\lambda+2) \alpha^{\prime}, \quad \mathrm{d}_{i}^{*} \alpha^{\prime}=0 .
$$

For $\lambda \neq-2,0$, solutions to (4.5) are equivalent to giving exact forms $\beta^{\prime} \in C^{\infty}\left(\Lambda^{3} T^{*} L_{i}\right)$ with $\Delta_{i} \beta^{\prime}=\lambda(\lambda+2) \beta^{\prime}$, where $\Delta_{i}$ is the Laplacian on $L_{i}$, and setting $\alpha^{\prime}=-(\lambda+2)^{-1} \mathrm{~d}_{i}^{*} \beta^{\prime}$. For $\lambda=0, \beta^{\prime}$ is locally constant and $\alpha^{\prime}=0$. Since $\beta$ is also an exact eigenform of $\Delta_{i}$ with eigenvalue $\lambda(\lambda+2)$ for $\lambda \neq 0$ and is constant if $\lambda=0$, we quickly deduce that $\beta=0$. Note that for the case $\lambda=-2, \beta$ must be zero since it is harmonic and exact. 
Observe that $\alpha$ is automatically orthogonal to coexact forms since $\mathrm{d}_{i} \alpha=0$ and $-(\lambda+2) \alpha=\mathrm{d}_{i} *_{i} \alpha$. Setting $\gamma_{i}=*_{i} \alpha$, we see that we can replace the quantities $d(\lambda)$ by $\check{d}(\lambda)$ in our estimate (4.2) for the dimension of $\mathcal{O}(N, \mu, \mathfrak{C})$ as claimed.

Theorem 3.14 and Proposition 4.11 invite us to define the stability index of a coassociative cone in a similar manner to [16, Definition 3.6].

Definition 4.12. Let $C$ be a coassociative cone in $\mathbb{R}^{7}$ with compact link $L \subseteq \mathcal{S}^{6}$ such that $C \backslash\{0\}$ is non-singular. Let $\mathcal{C}$ be a smooth, connected family of coassociative cones in $\mathbb{R}^{7}$ which contains $C$ and is closed under the natural action of $\mathrm{G}_{2} \ltimes \mathbb{R}^{7}$. Finally, for $\lambda \in \mathbb{R}$, let

$$
\check{D}(\lambda)=\left\{\gamma \in C^{\infty}\left(T^{*} L\right): \mathrm{d}^{*} \gamma=0, \mathrm{~d} \gamma=-(\lambda+2) * \gamma\right\}
$$

We define the $\mathcal{C}$-stability index of $C$ by

$$
\operatorname{ind}_{\mathcal{C}}(C)=\sum_{\lambda \in(-1,1]} \operatorname{dim} \check{D}(\lambda)-\operatorname{dim} \mathcal{C} .
$$

If the family $\mathcal{C}$ consists solely of the $\mathrm{G}_{2} \ltimes \mathbb{R}^{7}$ transformations of $C$, we simply write $\operatorname{ind}_{\mathcal{C}}(C)=\operatorname{ind}(C)$ and call ind $(C)$ the stability index of $C$.

Note that the sum in (4.7) is well-defined because the set of $\lambda$ for which $\operatorname{dim} \check{D}(\lambda) \neq 0$ is countable and discrete by the observations in Definition 3.12. Moreover, $\operatorname{dim} \check{D}(1)$ is the dimension of the space of Lagrangian Jacobi fields on $L$ by Definition 3.18, so the space of all infinitesimal deformations of $C$ as a coassociative cone, and $\check{D}(0)$ corresponds to the $O(1)$ closed self-dual 2 -forms on $C$, so $\operatorname{dim} \check{D}(0)$ is at least as large as the space of translations of $C$. Thus, $\operatorname{ind}_{\mathcal{C}}(C) \geq 0$.

We say that the cone $C$ is $\mathcal{C}$-stable if $\operatorname{ind}_{\mathcal{C}}(C)=0$. If $\operatorname{ind}(C)=0$ we say that the cone $C$ is stable. We also say that $C$ is rigid if $\operatorname{dim} \check{D}(1)=$ $14-\operatorname{dim} \mathrm{G}$, where $\mathrm{G}$ is the Lie subgroup of $\mathrm{G}_{2}$ preserving $C$.

\section{Notes.}

(a) It is clear that stability of $C$ implies rigidity. It is also clear that if $C$ is rigid then it is Jacobi integrable, since then every Lagrangian deformation of $L$ in $\mathcal{S}^{6}$ comes from $\mathrm{G}_{2}$ transformations. However, we shall see that one may have cones which are Jacobi integrable but neither stable nor rigid.

(b) By Theorem 3.14 and Proposition 4.11, if all the cones $C_{i}$ at the singularities of a CS coassociative 4 -fold $N$ are $\mathcal{C}_{i}$-stable and $\mu \in(1,2)$ is 
such that $(1, \mu] \cap \mathcal{D}=\emptyset$, then the obstruction space $\mathcal{O}(N, \mu, \mathfrak{C})=\{0\}$ and so the moduli space of deformations $\mathcal{M}(N, \mu, \mathfrak{C})$ is a smooth manifold near $N$. Moreover, $N$ is "stable" under deformations of the ambient closed $\mathrm{G}_{2}$ structure by Theorem 3.17.

We see from (4.6) that to determine the stability index for a coassociative cone we need to study the equation

$$
* \mathrm{~d} \gamma=-(\lambda+2) \gamma
$$

for 1 -forms $\gamma$ on a compact Riemannian 3-manifold $L$ for $\lambda \in(-1,1]$. The operator $* \mathrm{~d}: C^{\infty}\left(T^{*} L\right) \rightarrow C^{\infty}\left(T^{*} L\right)$ is a natural self-adjoint operator on $L$ which we call the curl operator. Thus, our problem is to calculate the negative eigenvalues of the curl operator and their multiplicities. For convenience we make the following definition.

Definition 4.13. Let $\left(L, g_{L}\right)$ be a compact Riemannian 3-manifold and let $c_{L}=-* \mathrm{~d}: C^{\infty}\left(T^{*} L\right) \rightarrow C^{\infty}\left(T^{*} L\right)$. Denote by $\sigma_{L}(\nu)$ the multiplicity of a non-zero eigenvalue $\nu$ of $c_{L}$. Note that $\operatorname{dim} \check{D}(\lambda)=\sigma_{L}(\lambda+2)$ for $\lambda \neq-2$.

Finding the positive spectrum of $c_{L}$ and the multiplicities is an extremely complicated problem and in general there is no hope to solve it. However, since we need only consider eigenvalues in $(1,3)$, the problem is tractable in special cases. For possible further applications to coassociative geometry it is of greatest practical use to study eigenvalues in the range $(0,4)$.

\section{Homogeneous cones}

In this section, we explicitly determine the stability index for coassociative cones whose links are orbits of closed 3-dimensional subgroups of $\mathrm{G}_{2}$. We achieve this by calculating the small eigenvalues of the curl operator on Berger 3-spheres and their quotients using elementary methods.

\subsection{Berger 3-spheres}

Definition 5.1. Let $\mathbb{H}$ denote the quaternions with standard basis $\{\mathbf{1}, \mathbf{i}, \mathbf{j}, \mathbf{k}\}$. Identify $\mathcal{S}^{3} \cong \operatorname{Sp}(1)$ and let $\mathbf{x}: \operatorname{Sp}(1) \rightarrow \mathbb{H}$ denote the inclusion map of $\operatorname{Sp}(1)$ as unit quaternions. Then $\mathrm{d} \mathbf{x}=\mathbf{x} \omega$ for a 1-form $\omega$ taking values in the Lie algebra of $\operatorname{Sp}(1)$, which here is represented by $\operatorname{Im} \mathbb{H}$. Therefore, 
we can write

$$
\mathbf{x}=x_{0} \mathbf{1}+x_{1} \mathbf{i}+x_{2} \mathbf{j}+x_{3} \mathbf{k} \quad \text { and } \quad \omega=\omega_{1} \mathbf{i}+\omega_{2} \mathbf{j}+\omega_{3} \mathbf{k}
$$

Since the Maurer-Cartan form $\omega$ satisfies the equations $\mathrm{d} \omega+\omega \wedge \omega=0$ and $\mathrm{d} \mathbf{x}=\mathbf{x} \omega$, we have that

$$
\begin{array}{ll}
\mathrm{d} x_{0}=-x_{1} \omega_{1}-x_{2} \omega_{2}-x_{3} \omega_{3}, & \mathrm{~d} x_{1}=x_{0} \omega_{1}-x_{3} \omega_{2}+x_{2} \omega_{3}, \\
\mathrm{~d} x_{2}=x_{3} \omega_{1}+x_{0} \omega_{2}-x_{1} \omega_{3}, & \mathrm{~d} x_{3}=-x_{2} \omega_{1}+x_{1} \omega_{2}+x_{0} \omega_{3}
\end{array}
$$

and

$$
\mathrm{d} \omega_{1}=-2 \omega_{2} \wedge \omega_{3}, \quad \mathrm{~d} \omega_{2}=-2 \omega_{3} \wedge \omega_{1}, \quad \mathrm{~d} \omega_{3}=-2 \omega_{1} \wedge \omega_{2}
$$

We define a 1-parameter family of metrics on $\mathcal{S}^{3}$ by $g_{\tau^{2}}=\tau^{2} \omega_{1}^{2}+\omega_{2}^{2}+\omega_{3}^{2}$ for $\tau>0$. The Riemannian manifolds $\left(\mathcal{S}^{3}, g_{\tau^{2}}\right)$ are the Berger 3-spheres.

It is immediately clear that finding the eigenvalues of $* \mathrm{~d}$ on a Berger 3 -sphere will involve the Laplacian acting on $\mathcal{S}^{3}$, thus homogeneous harmonic polynomials on $\mathbb{R}^{4}$. We are thus lead to make the following definitions.

Definition 5.2. Use the notation of Definition 5.1. For $m=1,2,3$, we define operators $\partial_{m}$ on $f \in C^{\infty}\left(\mathcal{S}^{3}\right)$ by the expression: $\mathrm{d} f=\partial_{1} f \omega_{1}+\partial_{2} f \omega_{2}$ $+\partial_{3} f \omega_{3}$. For a unit imaginary quaternion $\mathbf{q}=q_{1} \mathbf{i}+q_{2} \mathbf{j}+q_{3} \mathbf{k}$ we define $\partial_{\mathbf{q}}=$ $q_{1} \partial_{1}+q_{2} \partial_{2}+q_{3} \partial_{3}$. We say that $f \in C^{\infty}\left(\mathcal{S}^{3}\right)$ has $\mathbf{q}$-weight $w \geq 0$ if $\partial_{\mathbf{q}}^{2} f=$ $-w^{2} f$.

Observe that the Laplacian $\Delta_{\tau^{2}}$ on $\left(\mathcal{S}^{3}, g_{\tau^{2}}\right)$ acting on functions is given by $\Delta_{\tau^{2}}=-\frac{1}{\tau^{2}} \partial_{1}^{2}-\partial_{2}^{2}-\partial_{3}^{2}$.

Of course, $\partial_{m}$ is just Lie derivative along the vector field dual to $\omega_{m}$.

Definition 5.3. Use the notation of Definition 5.1. For $k \in \mathbb{N}$, let $\mathcal{P}_{k}$ be the space of homogeneous polynomials in $x_{0}, x_{1}, x_{2}, x_{3}$ of degree $k$ on $\mathcal{S}^{3}$. Let $\mathcal{Q}_{k}$ be the subspace of $\mathcal{P}_{k}$ consisting of polynomials which are eigenfunctions of the standard Laplacian $\Delta_{1}$; that is, restrictions of homogeneous harmonic polynomials in 4 real variables to $\mathcal{S}^{3}$. We let $\mathcal{A}_{k}=\left\{p_{1} \omega_{1}+p_{2} \omega_{2}+p_{3} \omega_{3}\right.$ : $\left.p_{1}, p_{2}, p_{3} \in \mathcal{P}_{k}\right\}$ and $\mathcal{B}_{k}=\left\{p_{1} \omega_{1}+p_{2} \omega_{2}+p_{3} \omega_{3}: p_{1}, p_{2}, p_{3} \in \mathcal{Q}_{k}\right\}$.

It is often clearer to work with the representation of $\mathrm{Sp}(1)=\mathrm{SU}(2)$ on $\mathbb{C}^{2}$ rather than $\mathbb{H}$, so we make the following useful definition. 
Definition 5.4. Recall the notation of Definition 5.1. We define complex coordinates on $\mathbb{H} \cong \mathbb{C}^{2}$ by $z_{1}=x_{0}+i x_{1}$ and $z_{2}=x_{2}+i x_{3}$. For $\mathbf{q}=q_{0} \mathbf{1}+$ $q_{1} \mathbf{i}+q_{2} \mathbf{j}+q_{3} \mathbf{k} \in \operatorname{Sp}(1)$, its action on $\mathbb{C}^{2}$ is given by

$$
\mathbf{q}:\left(\begin{array}{c}
z_{1} \\
z_{2}
\end{array}\right) \mapsto\left(\begin{array}{cc}
q_{0}+i q_{1} & q_{2}+i q_{3} \\
-q_{2}+i q_{3} & q_{0}-i q_{1}
\end{array}\right)\left(\begin{array}{c}
z_{1} \\
z_{2}
\end{array}\right) .
$$

For $k \in \mathbb{N}$, let $\mathcal{P}_{k}^{\mathbb{C}}$ denote the space of homogeneous polynomials in $z_{1}, z_{2}, \bar{z}_{1}$, $\bar{z}_{2}$ of degree $k$ restricted to $\mathcal{S}^{3}$ and let $\mathcal{Q}_{k}^{\mathbb{C}}$ be the subspace of $\mathcal{P}_{k}^{\mathbb{C}}$ consisting of polynomials which are harmonic on $\mathbb{C}^{2}$. We also let $\mathcal{R}_{m}^{\mathbb{C}}$, for $m \in \mathbb{Z}$, be the space of homogeneous polynomials $p$ in $z_{1}, z_{2}, \bar{z}_{1}, \bar{z}_{2}$, restricted to $\mathcal{S}^{3}$, such that under the action of $\cos \theta \mathbf{1}+\sin \theta \mathbf{i}, p$ maps to $e^{i m \theta} p$. Note that $p \in \mathcal{R}_{m}^{\mathbb{C}}$ if and only if $\bar{p} \in \mathcal{R}_{-m}^{\mathbb{C}}$.

We now recall the following well-known facts concerning eigenfunctions of the Laplacian on $\mathcal{S}^{3}$.

Theorem 5.5. Use the notation of Definitions 5.1-5.3.

(a) For each $k \in \mathbb{N}$, there is a direct sum decomposition $\mathcal{Q}_{k}=\oplus_{l=0}^{[k / 2]} \mathcal{Q}_{k, k-2 l}$ such that the elements of $\mathcal{Q}_{k, k-2 l}$ have $\mathbf{i}$-weight $k-2 l$. Moreover,

$$
\operatorname{dim} \mathcal{Q}_{k, k-2 l}=2 k+2 \text { if } l<\left[\frac{k}{2}\right], \quad \operatorname{dim} \mathcal{Q}_{2 l, 0}=2 l+1
$$

and $\operatorname{dim} \mathcal{Q}_{k}=(k+1)^{2}$.

(b) The eigenvalues of $\Delta_{\tau^{2}}$ are of the form $k(k+2)+\left(\frac{k-2 l}{\tau}\right)^{2}\left(1-\tau^{2}\right)$ for $k \in \mathbb{N}$ and $l \leq\left[\frac{k}{2}\right]$, and the corresponding eigenspace is $\mathcal{Q}_{k, k-2 l}$.

Proof. The results of [39, Lemmas 3.1 and 4.1] give the decomposition in (a) and all of (b). We can determine the dimensions of the $\mathcal{Q}_{k, k-2 l}$ by explicitly identifying the functions as the real and imaginary parts of elements in $\mathcal{Q}_{k}^{\mathbb{C}} \cap \mathcal{R}_{k-2 l}^{\mathbb{C}}$, in the notation of Definition 5.4 . Clearly $\mathcal{Q}_{2 l, 0}$ is the space of lifts of eigenfunctions of the Laplacian on $\mathbb{C P}^{1}$ with eigenvalue $4 l(l+1)$, which has dimension $2 l+1$. For the remaining spaces, it is straightforward to see that each $\mathcal{Q}_{k, k-2 l}$ has the same dimension for fixed $k$, and $\operatorname{since} \operatorname{dim} \mathcal{Q}_{k}=$ $(k+1)^{2}$, as it is the multiplicity of the eigenvalue $k(k+2)$ for $\Delta_{1}$ on $\mathcal{S}^{3}$, it is an elementary calculation to find that $\operatorname{dim} \mathcal{Q}_{k, k-2 l}=2 k+2$ if $l \neq\left[\frac{k}{2}\right]$.

The proofs of the quoted results from [39] rest on the fact that $\partial_{1}$ commutes with $\Delta_{\tau^{2}}$ for any $\tau>0$. This is certainly not true of $\partial_{2}$ and $\partial_{3}$ if $\tau \neq 1$. 
Proposition 5.6. Use the notation of Definitions 4.13 and 5.1 and Theorem 5.5. The positive eigenvalues of $c_{L}$ on $\left(L, g_{L}\right)=\left(\mathcal{S}^{3}, g_{\tau^{2}}\right)$ are

$$
\nu_{k, k-2 l}=\tau+\sqrt{\tau^{2}+k(k+2)+\left(\frac{k-2 l}{\tau}\right)^{2}\left(1-\tau^{2}\right)} \quad \text { and } \quad \nu_{k}=\frac{k+2}{\tau}
$$

for $k, l \in \mathbb{N}$ with $l \leq\left[\frac{k}{2}\right]$. Moreover,

$$
\sigma_{L}\left(\nu_{k, k-2 l}\right)=\operatorname{dim} \mathcal{Q}_{k, k-2 l} \quad \text { and } \quad \sigma_{L}\left(\nu_{k}\right)=2 k+2
$$

Note. For the multiplicity count here we regard the $\nu_{k, k-2 l}$ and $\nu_{k}$ as distinct. If they agree then we add the multiplicities.

Proof. From (5.1)-(5.3) we see that $c_{L}=-* \mathrm{~d}$ sends $\mathcal{A}_{k}$, given in Definition 5.3, to itself. Since $\cup_{k \in \mathbb{N}} \mathcal{A}_{k}$ is dense in $C^{\infty}\left(T^{*} L\right)$ we need only consider $c_{L} \alpha=\nu \alpha$ for $\alpha \in \mathcal{A}_{k}$ to determine the eigenvalues $\nu$ of $c_{L}$.

The equation $c_{L} \alpha=\nu \alpha$ for $\alpha=p_{1} \omega_{1}+p_{2} \omega_{2}+p_{3} \omega_{3}$ and $\nu>0$ is equivalent to the following system, using the notation of Definition 5.2:

$$
\begin{aligned}
& \left(2-\frac{\nu}{\tau}\right) p_{1}=\partial_{2} p_{3}-\partial_{3} p_{2}, \\
& (2-\nu \tau) p_{2}=\partial_{3} p_{1}-\partial_{1} p_{3}, \\
& (2-\nu \tau) p_{3}=\partial_{1} p_{2}-\partial_{2} p_{1} .
\end{aligned}
$$

Moreover, since $* \mathrm{~d} \alpha=-\nu \alpha$ for $\nu \neq 0$, we have that $\mathrm{d}^{*} \alpha=0$, which is equivalent to the condition:

$$
\frac{1}{\tau} \partial_{1} p_{1}+\tau \partial_{2} p_{2}+\tau \partial_{3} p_{3}=0
$$

From (5.1)-(5.2) we see that, if $\epsilon_{a b c}$ is the standard permutation symbol,

$$
\left[\partial_{a}, \partial_{b}\right]=2 \epsilon_{a b c} \partial_{c}
$$

Using (5.4)-(5.8) we calculate:

$$
\begin{aligned}
\Delta_{\tau^{2}} p_{1} & =-\left(\frac{1}{\tau^{2}} \partial_{1}^{2}+\partial_{2}^{2}+\partial_{3}^{2}\right) p_{1} \\
& =\left(\left[\partial_{1}, \partial_{2}\right]-(2-\nu \tau) \partial_{3}\right) p_{2}+\left(\left[\partial_{1}, \partial_{3}\right]+(2-\nu \tau) \partial_{2}\right) p_{3} \\
& =-\nu \tau\left(\partial_{2} p_{3}-\partial_{3} p_{2}\right) \\
& =\nu(\nu-2 \tau) p_{1} .
\end{aligned}
$$


Thus $p_{1}$ is a $\nu(\nu-2 \tau)$-eigenfunction of $\Delta_{\tau^{2}}$ if $p_{1} \neq 0$ and hence

$$
\nu(\nu-2 \tau)=k(k+2)+\left(\frac{k-2 l}{\tau}\right)^{2}\left(1-\tau^{2}\right),
$$

from which the formula for $\nu_{k, k-2 l}$ follows.

If $p_{1}=0$ then (5.4)-(5.8) imply that

$$
-\partial_{1}^{2} p_{j}=(\nu \tau-2)^{2} p_{j} \quad \text { and } \quad-\left(\partial_{2}^{2}+\partial_{3}^{2}\right) p_{j}=2(\nu \tau-2) p_{j},
$$

for $j=2,3$. Thus, $\Delta_{1} p_{j}=\nu \tau(\nu \tau-2) p_{j}$, so $\nu \tau(\nu \tau-2)=k(k+2)$ from which the formula for $\nu_{k}$ follows.

To determine the multiplicity of $\nu_{k, k-2 l}$ we make the following observations. First, using (5.5)-(5.6) we see that

$$
\begin{aligned}
& \left(\partial_{1}^{2}+(\nu \tau-2)^{2}\right) p_{2}=\left(\partial_{1} \partial_{2}-(\nu \tau-2) \partial_{3}\right) p_{1}, \\
& \left(\partial_{1}^{2}+(\nu \tau-2)^{2}\right) p_{3}=\left(\partial_{1} \partial_{3}+(\nu \tau-2) \partial_{2}\right) p_{1} .
\end{aligned}
$$

Second, from (5.4)-(5.7), we calculate

$$
\begin{aligned}
\left(\partial_{2}^{2}+\partial_{3}^{2}+2(\nu \tau-2)\right) p_{2} & =-\left(\frac{1}{\tau^{2}} \partial_{2} \partial_{1}+\left(4-\frac{\nu}{\tau}\right) \partial_{3}\right) p_{1}, \\
\left(\partial_{2}^{2}+\partial_{3}^{2}+2(\nu \tau-2)\right) p_{3} & =-\left(\frac{1}{\tau^{2}} \partial_{3} \partial_{1}-\left(4-\frac{\nu}{\tau}\right) \partial_{2}\right) p_{1} .
\end{aligned}
$$

Combining (5.12)-(5.15) we see that $p_{1}$ determines $p_{2}$ and $p_{3}$ unless they satisfy (5.11), which happens if and only if $\nu_{k, k-2 l}=\nu_{k}$. Thus, the multiplicity of $\sigma_{L}\left(\nu_{k, k-2 l}\right)$ is determined by the number of choices for $p_{1}$, so $\sigma_{L}\left(\nu_{k, k-2 l}\right)=\operatorname{dim} \mathcal{Q}_{k, k-2 l}$.

For the multiplicity of $\nu_{k}$, we may take $p_{1}=0$ and see that (5.6) determines $p_{3}$ given $p_{2}$ unless $k=0$. If $k \neq 0, \sigma_{L}\left(\nu_{k}\right)$ is the number of choices for $p_{2}$. By (5.11), we see that $p_{2} \in \mathcal{Q}_{k, k}$, so $\sigma_{L}\left(\nu_{k}\right)=\operatorname{dim} \mathcal{Q}_{k, k}=2 k+2$ by Theorem 5.5. For $k=0, p_{2}$ and $p_{3}$ are arbitrary constants so $\sigma_{L}\left(\nu_{0}\right)=2$.

Notes. We have some important elementary observations from the proof of Proposition 5.6, which will be important later.

(a) There is a basis for the $\nu_{k, k-2 l}$-eigenforms on $\left(\mathcal{S}^{3}, g_{\tau^{2}}\right)$ consisting of forms $p_{1} \omega_{1}+p_{2} \omega_{2}+p_{3} \omega_{3}$ with $p_{1} \in \mathcal{Q}_{k, k-2 l}$ and $p_{2}, p_{3} \in \mathcal{Q}_{k}$ determined by $p_{1}$.

(b) There is a basis for the $\nu_{k}$-eigenforms on $\left(\mathcal{S}^{3}, g_{\tau^{2}}\right)$ consisting of forms $p_{2} \omega_{2}+p_{3} \omega_{3}$ such that $p_{2}, p_{3} \in \mathcal{Q}_{k, k}$ and $p_{3}$ is determined by $p_{2}$ if $k>0$. 
We have an immediate corollary to Proposition 5.6 which builds on an already well-known result.

Corollary 5.7. Use the notation of Definitions 4.13 and 5.1. The positive eigenvalues of $c_{L_{0}}$ on $\left(L_{0}, g_{L_{0}}\right)=\left(\mathcal{S}^{3}, g_{1}\right)$ are $k+2$ for $k \in \mathbb{N}$ and we have $\sigma_{L_{0}}(k+2)=(k+2)^{2}-1$. Hence, a coassociative 4-plane in $\mathbb{R}^{7}$ is stable.

Proof. Clearly if $\tau=1$ then $\nu_{k, k-2 l}=1+\sqrt{(k+1)^{2}}=k+2=\nu_{k}$. If $k=$ $2 n$, then the multiplicity of the eigenvalue $2 n+2$ is

$$
\begin{aligned}
& \sum_{l=0}^{n-1} \sigma_{L_{0}}\left(\nu_{2 n, l}\right)+\sigma_{L_{0}}\left(\nu_{2 n, n}\right)+\sigma_{L_{0}}\left(\nu_{2 n}\right) \\
& \quad=n(4 n+2)+2 n+1+4 n+2=(2 n+2)^{2}-1 .
\end{aligned}
$$

Similarly, if $k=2 n+1$ the multiplicity of the eigenvalue $2 n+3$ is

$$
\sum_{l=0}^{n} \sigma_{L_{0}}\left(\nu_{2 n+1, l}\right)+\sigma_{L_{0}}\left(\nu_{2 n+1}\right)=(n+1)(4 n+4)+4 n+4=(2 n+3)^{2}-1
$$

The multiplicities follow.

For a coassociative 4-plane $C_{0}$,

$$
\sum_{\lambda \in(-1,1]} \operatorname{dim} \check{D}(\lambda)=\operatorname{dim} \check{D}(0)+\operatorname{dim} \check{D}(1)=\sigma_{L_{0}}(2)+\sigma_{L_{0}}(3)=3+8=11
$$

The number of non-trivial translations of $C_{0}$ is 3 , and the stabilizer of $C_{0}$ in $\mathrm{G}_{2}$ is isomorphic to $\mathrm{SO}(4)$ as observed in Example 4.1. Thus, the dimension of the space of $\mathrm{G}_{2} \ltimes \mathbb{R}^{7}$ transformations of $C_{0}$ is $3+14-6=11$. Therefore $\operatorname{ind}\left(C_{0}\right)=0$.

By [7, Example 5.1 and Theorem 5.1], the Lagrangian $L_{1}$ given in Example 4.2 is isometric to $\mathcal{S}^{3}$ with the metric $\frac{8}{3} g_{\frac{1}{6}}$. We may thus calculate the stability index of $C_{1}$ as follows.

Corollary 5.8. Use the notation of Definitions 4.13 and 5.1 and Example 4.2. The eigenvalues of $c_{L_{1}}$ on $\left(L_{1}, g_{L_{1}}\right)=\left(\mathcal{S}^{3}, \frac{8}{3} g_{\frac{1}{6}}\right)$ which lie in $(0,4)$ are 
given by $\left\{\frac{1}{2}, 2,3, \frac{1+\sqrt{145}}{4}, \frac{7}{2}\right\}$. Moreover,

$$
\begin{gathered}
\sigma_{L_{1}}\left(\frac{1}{2}\right)=1, \quad \sigma_{L_{1}}(2)=7, \quad \sigma_{L_{1}}(3)=10 \\
\sigma_{L_{1}}\left(\frac{1+\sqrt{145}}{4}\right)=5, \quad \sigma_{L_{1}}\left(\frac{7}{2}\right)=6 .
\end{gathered}
$$

Hence, the coassociative cone $C_{1}$ is stable.

Proof. Let $\lambda_{k, k-2 l}=\sqrt{\frac{3}{8}} \nu_{k, k-2 l}$ and let $\lambda_{k}=\sqrt{\frac{3}{8}} \nu_{k}$, using Proposition 5.6 with $\tau=\frac{1}{\sqrt{6}}$. Then $\lambda_{k, k-2 l}$ and $\lambda_{k}$ are the eigenvalues of $c_{L_{1}}$.

We notice that $\lambda_{5,1}=\frac{1}{4}(1+\sqrt{241})>4$, so we need only consider $\lambda_{k, k-2 l}$ for $k \leq 4$ for eigenvalues in $(0,4)$. We may calculate:

$$
\begin{aligned}
& \lambda_{0,0}=\frac{1}{2}, \quad \lambda_{1,1}=2, \quad \lambda_{2,2}=\frac{7}{2}, \quad \lambda_{2,0}=2, \quad \lambda_{3,3}=5, \quad \lambda_{3,1}=3, \\
& \lambda_{4,4}=\frac{13}{2}, \quad \lambda_{4,2}=\frac{1}{4}(1+\sqrt{265})>4, \quad \lambda_{4,0}=\frac{1}{4}(1+\sqrt{145})<4 .
\end{aligned}
$$

Since $\lambda_{k}=\frac{3 k}{2}+3$, only $\lambda_{0}=3$ is relevant here. By Proposition 5.6,

$$
\begin{aligned}
& \sigma_{L_{1}}\left(\frac{1}{2}\right)=\operatorname{dim} \mathcal{Q}_{0,0}, \\
& \sigma_{L_{1}}(2)=\operatorname{dim} \mathcal{Q}_{1,1}+\operatorname{dim} \mathcal{Q}_{2,0}, \quad \sigma_{L_{1}}(3)=\operatorname{dim} \mathcal{Q}_{3,1}+2, \\
& \sigma_{L_{1}}\left(\frac{1+\sqrt{145}}{4}\right)=\operatorname{dim} \mathcal{Q}_{4,0}, \quad \sigma_{L_{1}}\left(\frac{7}{2}\right)=\operatorname{dim} \mathcal{Q}_{2,2} .
\end{aligned}
$$

The multiplicities now follow from Theorem 5.5.

Since $C_{1}$ is non-planar and the stabilizer of $C_{1}$ under $\mathrm{G}_{2}$ transformations is $\mathrm{U}(2)$, the dimension of the family of $\mathrm{G}_{2} \ltimes \mathbb{R}^{7}$ transformations of $C_{1}$ is $7+14-4=17$. Further,

$$
\sum_{\lambda \in(-1,1]} \operatorname{dim} \check{D}(\lambda)=\operatorname{dim} \check{D}(0)+\operatorname{dim} \check{D}(1)=\sigma_{L_{1}}(2)+\sigma_{L_{1}}(3)=7+10=17,
$$

so $\operatorname{ind}\left(C_{1}\right)=0$.

Remark. We may observe, as in [40, Theorem 5], that $\tau^{2}=\frac{1}{6}$ is the critical value at which the multiplicity of the first eigenvalue of $\Delta_{\tau^{2}}$ on $\mathcal{S}^{3}$ "jumps". 


\subsection{Quotients of the 3-sphere}

We now wish to consider quotients of $\mathcal{S}^{3}$ by finite groups. The possible finite groups are the cyclic groups $\mathbb{Z}_{n}$, the binary dihedral groups $\mathrm{D}_{n}^{*}$, the binary tetrahedral group $\mathrm{A}_{4}^{*}$, the binary octahedral group $\mathrm{S}_{4}^{*}$ and the binary icosahedral group $\mathrm{A}_{5}^{*}$. We know, from Section 4.1, that the only groups we must consider are $\mathbb{Z}_{2}, \mathrm{D}_{3}^{*}$ and $\mathrm{A}_{4}^{*}$. We describe the actions of the finite subgroups we need explicitly.

Definition 5.9. Let unit quaternions act on $\mathbb{H}$ by left-multiplication.

(a) The cyclic group of order $n \geq 2$ acts as $\mathbb{Z}_{n}=\left\{\cos \left(\frac{2 k \pi}{n}\right) \mathbf{1}+\sin \left(\frac{2 k \pi}{n}\right) \mathbf{i}\right.$ : $k=0,1, \ldots, n-1\}$.

(b) The binary dihedral group of order $4 n$, for $n \geq 1$, acts as $\mathrm{D}_{n}^{*}=\mathbb{Z}_{2 n} \cup$ $\mathbf{j} \mathbb{Z}_{2 n}$.

(c) The binary tetrahedral group acts as $\mathrm{A}_{4}^{*}=\mathrm{D}_{2}^{*} \cup\left\{\frac{1}{2}( \pm \mathbf{1} \pm \mathbf{i} \pm \mathbf{j} \pm \mathbf{k})\right\}$, where any combination of signs is permissible.

To understand the spectrum of $c_{L}$ on quotients of $\mathcal{S}^{3}$ we need the following.

Proposition 5.10. Use the notation of Definitions 4.13 and 5.1. Let $\xi_{m}$ be the vector field on $\left(L, g_{L}\right)=\left(\mathcal{S}^{3}, g_{\tau^{2}}\right)$ dual to $\omega_{m}$. Then $c_{L}$ and the Lie derivative $\mathcal{L}_{\xi_{1}}$ commute for all $\tau>0$, and $c_{L}$ commutes with $\mathcal{L}_{\xi_{m}}$ for all $m$ if $\tau=1$.

Proof. Using Cartan's formula, we see that

$$
\left.\left.\left.\left[c_{L}, \mathcal{L}_{\xi_{1}}\right] \alpha=\xi_{1}\right\lrcorner \mathrm{~d} * \mathrm{~d} \alpha+\mathrm{d}\left(\xi_{1}\right\lrcorner * \mathrm{~d} \alpha\right)-* \mathrm{~d}\left(\xi_{1}\right\lrcorner \mathrm{d} \alpha\right)
$$

for 1 -forms $\alpha$. Let $\alpha=p_{1} \omega_{1}+p_{2} \omega_{2}+p_{3} \omega_{3}$ and let $\partial_{j}$ be the operator given in Definition 5.2 for $j=1,2,3$. We calculate:

$$
\begin{aligned}
* \mathrm{~d} \alpha= & \tau\left(\partial_{2} p_{3}-\partial_{3} p_{2}-2 p_{1}\right) \omega_{1} \\
& +\frac{1}{\tau}\left(\partial_{3} p_{1}-\partial_{1} p_{3}-2 p_{2}\right) \omega_{2}+\frac{1}{\tau}\left(\partial_{1} p_{2}-\partial_{2} p_{1}-2 p_{3}\right) \omega_{3},
\end{aligned}
$$


hence

$$
\begin{aligned}
\left.\xi_{1}\right\lrcorner \mathrm{d} * \mathrm{~d} \alpha= & \left(\left(\frac{1}{\tau} \partial_{1} \partial_{3}+2\left(\tau+\frac{1}{\tau}\right) \partial_{2}\right) p_{1}+\left(\tau \partial_{2} \partial_{3}-\frac{4}{\tau} \partial_{1}\right) p_{2}\right. \\
& \left.+\left(\frac{4}{\tau}-\frac{1}{\tau} \partial_{1}^{2}-\tau \partial_{2}^{2}\right) p_{3}\right) \omega_{2} \\
& +\left(\left(-\frac{1}{\tau} \partial_{1} \partial_{2}+2\left(\tau+\frac{1}{\tau}\right) \partial_{3}\right) p_{1}+\left(-\frac{4}{\tau}+\frac{1}{\tau} \partial_{1}^{2}+\tau \partial_{3}^{2}\right) p_{2}\right. \\
& \left.+\left(-\tau \partial_{3} \partial_{2}-\frac{4}{\tau} \partial_{1}\right) p_{3}\right) \omega_{3}
\end{aligned}
$$

and

$$
\begin{aligned}
\left.\mathrm{d}\left(\xi_{1}\right\lrcorner * \mathrm{~d} \alpha\right)= & \tau\left(-2 \partial_{1} p_{1}-\partial_{1} \partial_{3} p_{2}+\partial_{1} \partial_{2} p_{3}\right) \omega_{1} \\
& +\tau\left(-2 \partial_{2} p_{1}-\partial_{2} \partial_{3} p_{2}+\partial_{2}^{2} p_{3}\right) \omega_{2} \\
& +\tau\left(-2 \partial_{3} p_{1}-\partial_{3}^{2} p_{2}+\partial_{3} \partial_{2} p_{3}\right) \omega_{3}
\end{aligned}
$$

Since

$$
\left.\xi_{1}\right\lrcorner \mathrm{d} \alpha=\left(\partial_{1} p_{2}-\partial_{2} p_{1}-2 p_{3}\right) \omega_{2}-\left(\partial_{3} p_{1}-\partial_{1} p_{3}-2 p_{2}\right) \omega_{3},
$$

we have that:

$$
\begin{aligned}
\left.* \mathrm{~d}\left(\xi_{1}\right\lrcorner \mathrm{d} \alpha\right)= & \tau\left(\left[\partial_{3}, \partial_{2}\right] p_{1}+\left(-\partial_{3} \partial_{1}+2 \partial_{2}\right) p_{2}+\left(\partial_{2} \partial_{1}+2 \partial_{3}\right) p_{3}\right) \omega_{1} \\
& +\frac{1}{\tau}\left(\left(\partial_{1} \partial_{3}+2 \partial_{2}\right) p_{1}-4 \partial_{1} p_{2}+\left(4-\partial_{1}^{2}\right) p_{3}\right) \omega_{2} \\
& +\frac{1}{\tau}\left(\left(-\partial_{1} \partial_{2}+2 \partial_{3}\right) p_{1}-\left(4-\partial_{1}^{2}\right) p_{2}-4 \partial_{1} p_{3}\right) \omega_{3} .
\end{aligned}
$$

Combining these formulae and using (5.8) shows that $\left[c_{L}, \mathcal{L}_{\xi_{1}}\right] \alpha=0$. Clearly this argument will extend to $\mathcal{L}_{\xi_{2}}$ and $\mathcal{L}_{\xi_{3}}$ in the case $\tau=1$.

Proposition 5.11. Use the notation of Definitions 4.13 and 5.9 and Proposition 5.6. Let $n \in \mathbb{Z}^{+} \backslash\{1\}$. The positive eigenvalues of $c_{L}$ on $\left(L, g_{L}\right)=$ $\left(\mathcal{S}^{3} / \mathbb{Z}_{n}, g_{\tau^{2}}\right)$ are

$$
\begin{aligned}
\nu_{r n+2 l, r n} & =\tau+\sqrt{\tau^{2}+(r n+2 l)(r n+2 l+2)+\left(\frac{r n}{\tau}\right)^{2}\left(1-\tau^{2}\right)} \text { and } \\
\nu_{s n+n-2} & =\frac{(s+1) n}{\tau}
\end{aligned}
$$


for $l, r, s \in \mathbb{N}$. Moreover,

$$
\begin{gathered}
\sigma_{L}\left(\nu_{r n+2 l, r n}\right)=2 r n+4 l+2 \quad(r>0), \quad \sigma_{L}\left(\nu_{2 l, 0}\right)=2 l+1, \\
\sigma_{L}\left(\nu_{s n+n-2}\right)=2 s n+2 n-2 .
\end{gathered}
$$

Proof. Let $\xi_{1}$ be the vector field dual to $\omega_{1}$. By Proposition 5.10, $c_{L}$ and $\mathcal{L}_{\xi_{1}}$ commute. Thus, using the notation of Definition 5.3, we may restrict attention to $\alpha \in \mathcal{B}_{k} \otimes \mathbb{C}$ such that

$$
* \mathrm{~d} \alpha=-\nu \alpha \quad \text { and } \quad \mathcal{L}_{\xi_{1}} \alpha=i m \alpha
$$

for $\nu>0$ and $m \in \mathbb{Z}$. For $\alpha$ to descend to the quotient $\mathcal{S}^{3} / \mathbb{Z}_{n}$, we must have that $m \equiv 0(\bmod n)$. If we write $\alpha=p_{1} \omega_{1}+p_{2} \omega_{2}+p_{3} \omega_{3}$ then using Cartan's formula and the notation of Definition 5.2 we find that

$$
\begin{aligned}
i m \alpha & \left.\left.=\xi_{1}\right\lrcorner \mathrm{~d} \alpha+\mathrm{d}\left(\xi_{1}\right\lrcorner \alpha\right) \\
& \left.\left.=-\nu \xi_{1}\right\lrcorner * \alpha+\mathrm{d}\left(\xi_{1}\right\lrcorner\left(p_{1} \omega_{1}+p_{2} \omega_{2}+p_{3} \omega_{3}\right)\right) \\
& \left.=-\nu \xi_{1}\right\lrcorner\left(\frac{1}{\tau} p_{1} \omega_{2} \wedge \omega_{3}+\tau p_{2} \omega_{3} \wedge \omega_{1}+\tau p_{3} \omega_{1} \wedge \omega_{2}\right)+\mathrm{d} p_{1} \\
& =\partial_{1} p_{1} \omega_{1}+\left(\partial_{2} p_{1}-\nu \tau p_{3}\right) \omega_{2}+\left(\partial_{3} p_{1}+\nu \tau p_{2}\right) \omega_{3} .
\end{aligned}
$$

For $p_{1} \neq 0$ we see that $-\partial_{1}^{2} p_{1}=m^{2} p_{1}$, so $\alpha$ is a $\nu_{k, k-2 l}$-eigenform for $c_{L}$ by Proposition 5.6, where $k-2 l=r n$ for some $r \in \mathbb{N}$. For $p_{1}=0$, we observe that

$$
-m^{2} p_{2}=i m\left(i m p_{2}\right)=i m\left(-\nu \tau p_{3}\right)=-\nu^{2} \tau^{2} p_{2}
$$

Since $\nu \tau=k+2$ by Proposition 5.6, we have that $k=(s+1) n-2$ for some $s \in \mathbb{N}$, since $k \geq 0$. Thus $\alpha$ has eigenvalue $\nu_{s n+n-2}$ in these cases. The eigenvalues and multiplicities now follow from Theorem 5.5 and Proposition 5.6.

From the observations in [28, Example 6.14] we see that $L_{2}$ given in Example 4.3 is isometric to $\mathcal{S}^{3} / \mathbb{Z}_{2} \cong \mathrm{SO}(3)$ with metric $2 g_{2}$. We therefore calculate the spectrum of $c_{L_{2}}$ in $(0,4)$ using Proposition 5.11 as follows.

Corollary 5.12. Use the notation of Definitions 4.13, 5.1 and 5.9 and Example 4.3. The eigenvalues of $c_{L_{2}}$ on $\left(L_{2}, g_{L_{2}}\right)=\left(\mathcal{S}^{3} / \mathbb{Z}_{2}, 2 g_{2}\right)$ in $(0,4)$ 
are: $\{1,2,3,1+\sqrt{5}\}$. Moreover,

$$
\sigma_{L_{2}}(1)=2, \quad \sigma_{L_{2}}(2)=7, \quad \sigma_{L_{2}}(3)=16, \quad \sigma_{L_{2}}(1+\sqrt{5})=3
$$

Let $\mathcal{C}_{\circ}$ be the family of cones generated by $\mathrm{G}_{2} \ltimes \mathbb{R}^{7}$ transformations of cones

$$
C_{\mathbf{a}}=\left\{\left(0, z_{1}, z_{2}, z_{3}\right) \in \mathbb{R} \oplus \mathbb{C}^{3} \cong \mathbb{R}^{7}: a_{1} z_{1}^{2}+a_{2} z_{2}^{2}+a_{3} z_{3}^{2}=0\right\}
$$

where $\mathbf{a} \in \mathcal{T}=\left\{\left(a_{1}, a_{2}, a_{3}\right) \in \mathbb{R}^{3}: a_{i}>0\right.$ for all $i$ and $\left.a_{1}+a_{2}+a_{3}=1\right\}$. Then $C_{2}=C_{\left(\frac{1}{3}, \frac{1}{3}, \frac{1}{3}\right)}$, $\operatorname{ind}\left(C_{2}\right)=5$ and $\operatorname{ind}_{\mathcal{C}_{\circ}}\left(C_{2}\right)=0$, so $C_{2}$ is $\mathcal{C}_{\circ}$-stable and Jacobi integrable but not stable or rigid.

Proof. Using the notation of Proposition 5.6, let $\lambda_{2 r+2 l, 2 r}=\frac{1}{\sqrt{2}} \nu_{2 r+2 l, 2 r}$ and $\lambda_{2 s}=\frac{1}{\sqrt{2}} \nu_{2 s}$, calculated using $\tau=\sqrt{2}$. Then $\lambda_{2 r+2 l, 2 r}$ and $\lambda_{2 s}$ are the eigenvalues of $c_{L_{2}}$ by Proposition 5.11.

Since $\lambda_{4,4}=4$, we need only consider $\lambda_{2 r+2 l, 2 r}$ for $r, l \leq 1$. We also see that $\lambda_{2 s}=s+1$. We therefore calculate

$$
\begin{gathered}
\lambda_{0,0}=2, \quad \lambda_{2,2}=3, \quad \lambda_{2,0}=1+\sqrt{5}, \\
\lambda_{0}=1, \quad \lambda_{2}=2, \quad \lambda_{4}=3 .
\end{gathered}
$$

The eigenvalues and multiplicities follow from Proposition 5.11.

Since $C_{2}$ is non-planar and the stabilizer of $C_{2}$ under $\mathrm{G}_{2}$ transformations is $\mathrm{SO}(3)$, the dimension of the family of $\mathrm{G}_{2} \ltimes \mathbb{R}^{7}$ transformations of $C_{2}$ is $7+14-3=18$. Further,

$\sum_{\lambda \in(-1,1]} \operatorname{dim} \check{D}(\lambda)=\operatorname{dim} \check{D}(0)+\operatorname{dim} \check{D}(1)=\sigma_{L_{2}}(2)+\sigma_{L_{2}}(3)=7+16=23$

so ind $\left(C_{2}\right)=5$. Now, $C_{2}=C_{\left(\frac{1}{3}, \frac{1}{3}, \frac{1}{3}\right)} \in \mathcal{C}_{\circ}$. Moreover, if $\mathbf{a} \neq\left(\frac{1}{3}, \frac{1}{3}, \frac{1}{3}\right), C_{\mathbf{a}}$ has trivial stabilizer in $\mathrm{G}_{2} \ltimes \mathbb{R}^{7}$ and has a two-parameter family of deformations up to rigid motion given by varying a. Therefore, $\operatorname{dim} \mathcal{C}_{\circ}=7+14+2=$ 23 and $C_{2}$ is $\mathcal{C}_{\circ}$-stable. Finally note that $\operatorname{dim} \check{D}(1)=16$, so the space of Lagrangian Jacobi fields on $L_{2}$ is equal to the space of genuine deformations of $L_{2}$ in the family of links of cones in $\mathcal{C}_{\circ}$, which also shows that $\mathcal{C}_{\circ}$ is a maximal deformation family for $C_{2}$. 
We now consider the binary dihedral group.

Proposition 5.13. Use the notation of Definitions 4.13, 5.1 and 5.9 and Proposition 5.6. For $n \in \mathbb{Z}^{+}$, the positive eigenvalues of $c_{L}$ on $\left(L, g_{L}\right)=$ $\left(\mathcal{S}^{3} / \mathrm{D}_{n}^{*}, g_{\tau^{2}}\right)$ are:

$$
\begin{aligned}
& \nu_{2 r n+2 n+2 l, 2 r n+2 n} \\
& \quad=\tau+\sqrt{\tau^{2}+4(r n+n+l)(r n+n+l+1)+4\left(\frac{r n+n}{\tau}\right)^{2}\left(1-\tau^{2}\right)} ; \\
& \nu_{4 l, 0}=\tau+\sqrt{\tau^{2}+8 l(2 l+1)} ; \quad \text { and } \quad \nu_{2 s n+2 n-2}=\frac{2(s+1) n}{\tau}
\end{aligned}
$$

for $l, r, s \in \mathbb{N}$. Moreover,

$$
\begin{gathered}
\sigma_{L}\left(\nu_{2 r n+2 n+2 l, 2 r n+2 n}\right)=2 r n+2 n+2 l+1, \quad \sigma_{L}\left(\nu_{4 l, 0}\right)=4 l+1, \\
\sigma_{L}\left(\nu_{2 s n+2 n-2}\right)=4 s n+4 n-2 .
\end{gathered}
$$

Proof. Let $\alpha=p_{1} \omega_{1}+p_{2} \omega_{2}+p_{3} \omega_{3}$ be an eigenform of $c_{L}$ of positive eigenvalue $\nu$. From Definition 5.9(b), $\mathbb{Z}_{2 n} \subseteq \mathrm{D}_{n}^{*}$. Hence, by Proposition 5.11, the possible $\nu$ are of the form $\nu_{k, k-2 l}$ where $k-2 l \equiv 0(\bmod 2 n)$ or $\nu_{2 s n+2 n-2}$, using the notation of Proposition 5.6.

Recalling Definition 5.4, we see that $\mathbf{j}$ sends $\left(z_{1}, z_{2}\right) \in \mathbb{C}^{2}$ to $\left(z_{2},-z_{1}\right)$ and hence $\mathbf{j}$ maps $\mathcal{R}_{m}^{\mathbb{C}}$ to $\mathcal{R}_{-m}^{\mathbb{C}}$ for each $m \in \mathbb{Z}$. By note (a) after Proposition 5.6 and Definition 5.9(b), the multiplicity of $\nu_{2 r n+2 l, 2 r n}$ is the number of choices of $p_{1} \in \mathcal{Q}_{2 r n+2 l, 2 r n}$ which are $\mathbf{j}$-invariant. By Theorem 5.5, $p_{1}$ is the sum of real and imaginary parts of polynomials in $\mathcal{Q}_{2 r n+2 l}^{\mathbb{C}} \cap \mathcal{R}_{2 r n}^{\mathbb{C}}$. Since $\mathbf{j}^{2}$ clearly acts as the identity on $\mathcal{Q}_{2 r n+2 l}^{\mathbb{C}}$, we can decompose $\mathcal{Q}_{2 r n+2 l, 2 r n}$ into \pm 1 -eigenspaces for $\mathbf{j}$. Thus, for $r>0$, the subspace of $\mathcal{Q}_{2 r n+2 l, 2 r n}$ which is $\mathbf{j}$-invariant is half the total dimension. For $r=0$, it is straightforward to see that $\mathbf{j}$ acts as $(-1)^{l}$ on $\mathcal{Q}_{2 l}^{\mathbb{C}} \cap \mathcal{R}_{0}^{\mathbb{C}}$, so $p_{1} \in \mathcal{Q}_{2 l, 0}$ is $\mathbf{j}$-invariant if and only if $l$ is even.

By note (b) after Proposition 5.6, the multiplicity of $\nu_{k}$, if $k \neq 0$, is determined by the number of choices for $p_{2}$. Now, since $p_{2}$ is only welldefined up to sign on the quotient of $\mathcal{S}^{3}$ by $\mathrm{D}_{n}^{*}$, we need to calculate the number of polynomials in $\mathcal{Q}_{k, k}$ on which $\mathbf{j}$ acts as \pm 1 . However, since $k=$ $2 s n+2 n-2>0$ is even, we can find a basis for $\mathcal{Q}_{k, k}$ consisting of \pm 1 eigenfunctions for $\mathbf{j}$ by the previous paragraph. For $\nu_{0}$ the multiplicity is 2 because $p_{2}$ and $p_{3}$ are constant.

The result follows from Theorem 5.5 and Proposition 5.6. 
By [28, Example 6.15], $L_{3}$ given in Example 4.4 is isometric to $\mathrm{SO}(3) /$ $\mathrm{S}_{3} \cong \mathcal{S}^{3} / \mathrm{D}_{3}^{*}$ with metric $6 g_{6}$. We may thus apply Proposition 5.13 as follows.

Corollary 5.14. Use the notation of Definitions 4.13, 5.1 and 5.9 and Example 4.4. The eigenvalues of $c_{L_{3}}$ on $\left(L_{3}, g_{L_{3}}\right)=\left(\mathcal{S}^{3} / \mathrm{D}_{3}^{*}, 6 g_{6}\right)$ in $(0,4)$ are: $\{1,2,3,1+\sqrt{5}\}$. Moreover,

$$
\sigma_{L_{3}}(1)=10, \quad \sigma_{L_{3}}(2)=23, \quad \sigma_{L_{3}}(3)=41, \quad \sigma_{L_{3}}(1+\sqrt{5})=5 .
$$

Hence, the cone $C_{3}$ is not stable and $\operatorname{ind}\left(C_{3}\right)=46$. Moreover, $C_{3}$ is not rigid.

Proof. By Proposition 5.13 applied with $\tau=\sqrt{6}$, the eigenvalues of $c_{L_{3}}$ are $\lambda_{6 r+6+2 l, 6 r+6}=\frac{1}{\sqrt{6}} \nu_{6 r+6+2 l, 6 r+6}, \quad \lambda_{4 l, 0}=\frac{1}{\sqrt{6}} \nu_{4 l, 0}$ and $\lambda_{6 s+4}=\frac{1}{\sqrt{6}} \nu_{6 s+4}$, for $l, r, s \in \mathbb{N}$. Since $\lambda_{8,0}=1+\frac{1}{3} \sqrt{129}>4, \lambda_{8,6}=1+\frac{1}{3} \sqrt{84}>4$ and $\lambda_{12,12}=4$, we need only calculate:

$$
\lambda_{0,0}=2, \quad \lambda_{4,0}=1+\sqrt{5}, \quad \lambda_{6,6}=3, \quad \lambda_{6 s+4}=s+1 .
$$

The multiplicities now follow from Proposition 5.13.

Since $C_{3}$ is non-planar and has $\mathrm{SO}(3)$ stabilizer in $\mathrm{G}_{2}$,

$$
\begin{aligned}
\operatorname{ind}\left(C_{3}\right) & =\sum_{\lambda \in(-1,1]} \operatorname{dim} \check{D}(\lambda)-7-(14-3)=\operatorname{dim} \check{D}(0)+\operatorname{dim} \check{D}(1)-18 \\
& =\sigma_{L_{3}}(2)+\sigma_{L_{3}}(3)-18=23+41-18=46
\end{aligned}
$$

as claimed. We also see that $\operatorname{dim} \check{D}(1)=41>11=\operatorname{dim} \mathrm{G}_{2}-\operatorname{dim} \mathrm{SO}(3)$, so $C_{3}$ is not rigid.

Lastly, we study the constant curvature 1 metric on $\mathcal{S}^{3} / \mathrm{A}_{4}^{*}$.

Proposition 5.15. Use the notation of Definitions 4.13 and 5.9. Let $\left(L, g_{L}\right)=\left(\mathcal{S}^{3} / \mathrm{A}_{4}^{*}, g_{1}\right)$. The positive eigenvalues of $c_{L}$ are

$$
\nu_{2 r}=2 r+2 \quad \text { and } \quad \nu_{6 s+4,6 s+4}=6 s+6
$$

for $r, s \in \mathbb{N}$. Moreover,

$$
\sigma_{L}\left(\nu_{2 r}\right)=(2 r+1)\left(1+2\left[\frac{r}{3}\right]+\left[\frac{r}{2}\right]-r\right) \quad \text { and } \quad \sigma_{L}\left(\nu_{6 s+4,6 s+4}\right)=12 s+10
$$

where $[q]$ denotes the integer part of a non-negative rational number $q$. 
Proof. From Definition 5.9(c), we observe that $\mathrm{A}_{4}^{*}$ is generated by $\mathbf{i}$ and $\mathbf{j}$, which are elements of order 4 , and

$$
\frac{1}{2}(\mathbf{1}+\mathbf{i}+\mathbf{j}+\mathbf{k})=\frac{1}{2} \mathbf{1}+\frac{\sqrt{3}}{2}\left(\frac{\mathbf{i}+\mathbf{j}+\mathbf{k}}{\sqrt{3}}\right),
$$

which has order 6. By Proposition 5.6 and Corollary 5.7, we know that the possible positive eigenvalues of $c_{L}$ are $k+2$ for $k \in \mathbb{N}$ with corresponding eigenforms $\alpha \in \mathcal{B}_{k}$, in the notation of Definition 5.3.

Suppose $\alpha=p_{1} \omega_{1}+p_{2} \omega_{2}+p_{3} \omega_{3} \in \mathcal{B}_{k} \otimes \mathbb{C}$ is an eigenform of $c_{L}$. We may write $\alpha=P_{1} \Omega_{1}+P_{2} \Omega_{2}+P_{3} \Omega_{3}$ where

$$
\begin{array}{lll}
\Omega_{1}=\frac{\omega_{1}+\omega_{2}+\omega_{3}}{\sqrt{3}}, & \Omega_{2}=\frac{\omega_{1}-\omega_{3}}{\sqrt{2}}, & \Omega_{3}=\frac{-\omega_{1}+2 \omega_{2}-\omega_{3}}{\sqrt{6}}, \\
P_{1}=\frac{p_{1}+p_{2}+p_{3}}{\sqrt{3}}, & P_{2}=\frac{p_{1}-p_{3}}{\sqrt{2}}, & P_{3}=\frac{-p_{1}+2 p_{2}-p_{3}}{\sqrt{6}} .
\end{array}
$$

Let $\xi_{m}$ be the vector field dual to $\omega_{m}$ and set $\xi=\left(\xi_{1}+\xi_{2}+\xi_{3}\right) / \sqrt{3}$. Since $\xi$ commutes with $c_{L}$ by Proposition 5.10, we may look for $\alpha$ satisfying

$$
c_{L} \alpha=(k+2) \alpha \quad \text { and } \quad \mathcal{L}_{\xi} \alpha=i m \alpha
$$

for $m \in \mathbb{Z}$. For $\alpha$ to be well-defined on $L$ we must have that $m \equiv 0(\bmod 6)$. Using (5.3) we calculate:

$$
\begin{aligned}
\mathcal{L}_{\xi} \Omega_{1} & =\frac{1}{3}\left(\mathcal{L}_{\xi_{2}+\xi_{3}} \omega_{1}+\mathcal{L}_{\xi_{3}+\xi_{1}} \omega_{2}+\mathcal{L}_{\xi_{1}+\xi_{2}} \omega_{3}\right) \\
& =\frac{1}{3}\left(-2 \omega_{3}+2 \omega_{2}-2 \omega_{1}+2 \omega_{3}-2 \omega_{2}+2 \omega_{1}\right)=0 ; \\
\mathcal{L}_{\xi} \Omega_{2} & =2 \Omega_{3} ; \\
\mathcal{L}_{\xi} \Omega_{3} & =-2 \Omega_{2} .
\end{aligned}
$$

Recall the notation of Definitions 5.2 and 5.4. By similar methods to the proof of Proposition 5.11, $P_{1}$ has q-weight $6 l$ for some $l \in \mathbb{N}$, where $\mathbf{q}=\frac{\mathbf{i}+\mathbf{j}+\mathbf{k}}{\sqrt{3}}$. Moreover, $P_{1}$ must be invariant under $\mathbf{i}$ and $\mathbf{j}$, so $P_{1}$ is $\mathrm{A}_{4^{-}}^{*}$ invariant. The $\mathrm{A}_{4}^{*}$-invariant eigenfunctions of the Laplacian on $\mathcal{S}^{3}$ are effectively determined in [12, Theorem 4.4], so we deduce that $k=2 r$ and $P_{1}$ lies in a subspace of $\mathcal{Q}_{2 r}^{\mathbb{C}}$ of dimension $(2 r+1)\left(1+2\left[\frac{r}{3}\right]+\left[\frac{r}{2}\right]-r\right)$. The formulae for $\nu_{2 r}$ and $\sigma_{L}\left(\nu_{2 r}\right)$ follow.

Again following the proof of Proposition 5.11, we observe that $P_{1}$ determines $P_{2}$ and $P_{3}$ unless $P_{2}, P_{3} \in \mathcal{Q}_{6 s+4}^{\mathbb{C}}$ have q-weight $6 s+4$ for some $s \in \mathbb{N}$. 
Thus, we also have eigenvalues of the form $6 s+6$ with multiplicity equal to the number of choices for $P_{2}$. As in the proof of Proposition 5.13, $P_{2}$ is only well-defined up to sign on the quotient of $\mathcal{S}^{3}$. Since $P_{2}$ is a polynomial of even degree it has even $\mathbf{i}$-weight and $\mathbf{j}$-weight, so $P_{2}$ maps to $\pm P_{2}$ under the action of $\mathbf{i}$ and $\mathbf{j}$. Since we may decompose the harmonic polynomials on $\mathcal{S}^{3}$ by $\mathbf{q}$-weight rather than $\mathbf{i}$-weight as in Theorem 5.5, and the metric on $L$ has constant curvature, we deduce that $\sigma_{L}(6 s+6)=\operatorname{dim} \mathcal{Q}_{6 s+4,6 s+4}=$ $12 s+10$.

As observed in [28, Example 6.6], the link $L_{4}$ of $C_{4}$, given in Example 4.4, is isometric to $\mathrm{SO}(3) / \mathrm{A}_{4} \cong \mathcal{S}^{3} / \mathrm{A}_{4}^{*}$ with constant curvature $\frac{1}{16}$. We may therefore deduce the following.

Proposition 5.16. Use the notation of Definitions 4.13, 5.1 and 5.9 and Example 4.4. The eigenvalues of $c_{L_{4}}$ on $\left(L_{4}, g_{L_{4}}\right)=\left(\mathcal{S}^{3} / \mathrm{A}_{4}^{*}, 16 g_{1}\right)$ in $(0,4)$ are: $\left\{\frac{1}{2}, \frac{3}{2}, 2, \frac{5}{2}, 3, \frac{7}{2}\right\}$. Moreover,

$$
\begin{aligned}
& \sigma_{L_{4}}\left(\frac{1}{2}\right)=1, \quad \sigma_{L_{4}}\left(\frac{3}{2}\right)=10, \quad \sigma_{L_{4}}(2)=7, \\
& \sigma_{L_{4}}\left(\frac{5}{2}\right)=9, \quad \sigma_{L_{4}}(3)=22, \quad \sigma_{L_{4}}\left(\frac{7}{2}\right)=26 .
\end{aligned}
$$

Hence, the cone $C_{4}$ is not stable and $\operatorname{ind}\left(C_{4}\right)=30$. Moreover, $C_{4}$ is not rigid.

Proof. By Proposition 5.15, the positive eigenvalues of $c_{L_{4}}$ are $\lambda_{2 r}=\frac{1}{2}(r+1)$ and $\lambda_{6 s+4,6 s+4}=\frac{3}{2}(s+1)$. We are restricted to $r=0,3,4,6$ and $s=0,1$ for eigenvalues in $(0,4)$ since the multiplicity of $\lambda_{2 r}$ for $r=1,2,5$ is zero.

Hence, on $C_{4}$,

$$
\begin{aligned}
\sum_{\lambda \in(-1,1]} \operatorname{dim} \check{D}(\lambda) & =\operatorname{dim} \check{D}\left(-\frac{1}{2}\right)+\operatorname{dim} \check{D}(0)+\operatorname{dim} \check{D}\left(\frac{1}{2}\right)+\operatorname{dim} \check{D}(1) \\
& =\sigma_{L_{4}}\left(\frac{3}{2}\right)+\sigma_{L_{4}}(2)+\sigma_{L_{4}}\left(\frac{5}{2}\right)+\sigma_{L_{4}}(3) \\
& =10+7+9+22=48 .
\end{aligned}
$$

Since $C_{4}$ is non-planar and $\mathrm{SO}(3)$-invariant, the dimension of the family of $\mathrm{G}_{2} \ltimes \mathbb{R}^{7}$ transformations of $C_{4}$ has dimension $7+14-3=18$. Thus $\operatorname{ind}\left(C_{4}\right)=30$. Observe that $\operatorname{dim} \check{D}(1)=22>11=\operatorname{dim} \mathrm{G}_{2}-\operatorname{dim} \mathrm{SO}(3)$, so $C_{4}$ is not rigid. 
The stability index is a measure of the geometry complexity of the coassociative cone. We might therefore expect homogeneous cones to have the greatest chance of being stable. Since we find that the only stable homogeneous coassociative cones are 4-planes and the cone in Example 4.2, we might naively expect that these are the only stable coassociative cones.

In Geometric Measure Theory one defines the $m$-dimensional density of a set $S \subseteq \mathbb{R}^{n}$ at a point $p$ by

$$
\Theta(S, p)=\lim _{r \rightarrow 0} \frac{\mathcal{H}^{m}\left(S \cap B_{n}(p ; r)\right)}{\operatorname{vol}\left(B_{m}(0 ; r)\right)},
$$

where $\mathcal{H}^{m}$ is $m$-dimensional Hausdorff measure and $B_{n}(p ; r)$ is the ball in $\mathbb{R}^{n}$ of radius $r$ about $p$. If $S$ is an $m$-dimensional submanifold then $\Theta(S, p)=1$ for all $p \in S$. For coassociative cones $C$ in $\mathbb{R}^{7}$ with isolated singularities at the origin and compact links $L$ it is straightforward to calculate the 4-dimensional density as $\Theta(C, 0)=\operatorname{vol}(L) / \operatorname{vol}\left(\mathcal{S}^{3}\right)$. We may therefore easily calculate the density for the homogeneous coassociative cones given in Examples 4.1-4.4 as follows:

$\Theta\left(C_{0}, 0\right)=1, \Theta\left(C_{1}, 0\right)=\frac{16}{9}, \Theta\left(C_{2}, 0\right)=4, \Theta\left(C_{3}, 0\right)=36, \Theta\left(C_{4}, 0\right)=64$.

The fact that $C_{0}$ and $C_{1}$ are the homogeneous cones with the lowest density and are also the only stable ones is suggestive.

\section{Algebraic curves and 2-ruled cones}

Since Example 4.8 gives the largest known family of coassociative cones, we are motivated to analyse the stability index for 2-ruled cones. We therefore need to understand the curl operator on geodesic $\mathcal{S}^{1}$-bundles over surfaces. We are particularly interested in the case where the surface is an algebraic curve.

We start with some definitions from the theory of Riemannian submersions.

Definition 6.1. Let $\left(L, g_{L}\right)$ be a compact Riemannian 3-manifold which is an $\mathcal{S}^{1}$-bundle $\pi: L \rightarrow \Sigma$ over a compact Riemannian surface $\left(\Sigma, g_{\Sigma}\right)$. Suppose further that $\pi$ is a Riemannian submersion. Let $\xi$ be a unit vector field spanning the vertical distribution of $\pi$ and let $\theta$ be the 1 -form dual to $\xi$.

We define a form $\alpha$ on $L$ to be horizontal if $\xi\lrcorner \alpha=0$ and we denote the bundle of horizontal $m$-forms by $\Lambda_{h}^{m} T^{*} L$. Trivially all functions are horizontal. We can identify the horizontal $m$-forms with $m$-forms on $\Sigma$. We 
define the horizontal Hodge star $*_{h}$ on horizontal forms $\alpha$ via the equation: $* \alpha=*_{h} \alpha \wedge \theta$.

By [33, Equation (2.1.2)],

$$
\Lambda^{m+1} T^{*} L=\Lambda_{h}^{m+1} T^{*} L \oplus\left(\Lambda_{h}^{m} T^{*} L \wedge \theta\right),
$$

so we can define a horizontal derivative $\mathrm{d}_{h}: C^{\infty}\left(\Lambda^{m} T^{*} L\right) \rightarrow C^{\infty}\left(\Lambda_{h}^{m+1} T^{*} L\right)$ by sending $\alpha$ to the horizontal part of $\mathrm{d} \alpha$. Since $\mathrm{d}_{h}$ sends any section of $\Lambda_{h}^{m} T^{*} L \wedge \theta$ to zero, $\mathrm{d}_{h}: C^{\infty}\left(\Lambda_{h}^{m} T^{*} L\right) \rightarrow C^{\infty}\left(\Lambda_{h}^{m+1} T^{*} L\right)$ is an antiderivation. We can therefore define the formal adjoint $\mathrm{d}_{h}^{*}$ of $\mathrm{d}_{h}$ and the horizontal Laplacian $\Delta_{h}=\mathrm{d}_{h} \mathrm{~d}_{h}^{*}+\mathrm{d}_{h}^{*} \mathrm{~d}_{h}$. The horizontal Laplacian is not necessarily elliptic.

If $\Delta_{L}$ is the ordinary Laplacian on $L$, we call $\Delta_{v}=\Delta_{L}-\Delta_{h}$ the vertical Laplacian. Note in the case of functions that $\Delta_{v}=-\mathcal{L}_{\xi}^{2}$.

The situation above includes the Berger 3-spheres, tubes over pseudoholomorphic curves in $\mathcal{S}^{6}$ and real links of complex 2-dimensional cones.

The key result for understanding the Laplacian on functions in the situation of Definition 6.1 is the following [3, Theorem 1.5].

Proposition 6.2. Use the notation of Definition 6.1. If $\pi: L \rightarrow \Sigma$ has totally geodesic fibres then, on functions, $\Delta_{L}, \Delta_{h}$ and $\Delta_{v}$ commute.

For convenience we make the following definition.

Definition 6.3. Let $L$ be a compact Riemannian 3-manifold. Let $m_{L}(\nu)$ denote the multiplicity of the eigenvalue $\nu$ of the Laplacian $\Delta_{L}$ on functions. Let $\mathcal{E}_{L}=\left\{\nu \in \mathbb{R}: m_{L}(\nu) \neq 0\right\}$, which is a countable discrete set.

Remarks. Use the notation of Definition 6.1 and suppose that $\pi: L \rightarrow$ $\Sigma$ has totally geodesic fibres. Let $m_{L}\left(\nu_{h}, \nu_{v}\right)$ denote the dimension of the space of functions $f$ such that $\Delta_{h} f=\nu_{h} f$ and $\Delta_{v} f=\nu_{v} f$. Note that, since $\Delta_{h}$ and $\Delta_{v}$ are non-negative operators, we must have $\nu_{h} \geq 0$ and $\nu_{v} \geq 0$. Moreover, by one of the main results in [42], $m_{L}\left(\nu_{h}, 0\right)$ is the multiplicity of the eigenvalue $\nu_{h}$ of the Laplacian $\Delta_{\Sigma}$ acting on functions on $\Sigma$. Finally, by Proposition 6.2, every eigenvalue of $\Delta_{L}$ is of the form $\nu_{h}+\nu_{v}$, so $m_{L}(\nu) \leq$ $\sum_{\nu_{h}+\nu_{v}=\nu} m_{L}\left(\nu_{h}, \nu_{v}\right)$.

We now have the following result that is similar to Proposition 5.10. 
Proposition 6.4. Use the notation of Definitions 4.13 and 6.1. If the fibres of $\pi: L \rightarrow \Sigma$ are totally geodesic and $\xi$ is a Killing vector field for $g_{L}$, then $c_{L}$ and the Lie derivative $\mathcal{L}_{\xi}$ commute.

Proof. Recall Definition 6.1. By the work in [33, Section 2.1], since $\nabla_{\xi} \xi=0$, $\mathrm{d} \theta$ is horizontal. Moreover, if we write a 1-form $\gamma$ on $L$ as $\gamma=\alpha+f \theta$ with $\alpha$ horizontal and $f$ a function, then

$$
* \mathrm{~d} \gamma=*_{h}\left(\mathcal{L}_{\xi} \alpha-\mathrm{d}_{h} f\right)+\left(*_{h} \mathrm{~d}_{h} \alpha+f *_{h} \mathrm{~d} \theta\right) \theta \quad \text { and } \quad \mathcal{L}_{\xi} \gamma=\mathcal{L}_{\xi} \alpha+\left(\mathcal{L}_{\xi} f\right) \theta
$$

Thus, since $\xi$ is Killing and $\mathrm{d} \theta$ is horizontal, it is straightforward to compute:

$$
* \mathrm{~d}\left(\mathcal{L}_{\xi} \gamma\right)=\left({ }_{h} \mathcal{L}_{\xi}^{2} \alpha-*_{h} \mathrm{~d}_{h}\left(\mathcal{L}_{\xi} f\right)\right)+\left({ }{ }_{h} \mathrm{~d}_{h}\left(\mathcal{L}_{\xi} \alpha\right)+\left(\mathcal{L}_{\xi} f\right) *_{h} \mathrm{~d} \theta\right) \theta
$$

and

$$
\mathcal{L}_{\xi}(* \mathrm{~d} \gamma)=\left(*_{h} \mathcal{L}_{\xi}^{2} \alpha-*_{h} \mathcal{L}_{\xi}\left(\mathrm{d}_{h} f\right)\right)+\left(*_{h} \mathcal{L}_{\xi}\left(\mathrm{d}_{h} \alpha\right)+\left(\mathcal{L}_{\xi} f\right) *_{h} \mathrm{~d} \theta\right) \theta
$$

Note that, since the fibres of $\pi$ are totally geodesic, $\left[\mathrm{d}_{h}, \mathcal{L}_{\xi}\right]=0$ on horizontal forms by $\left[33\right.$, Equation (2.1.6)]. We conclude that $\left[c_{L}, \mathcal{L}_{\xi}\right]=0$ as claimed.

Observe that, by Example 4.9, we have two types of links of coassociative cones where Definition 6.1 and Propositions 6.2 and 6.4 apply: namely, Hopf lifts of holomorphic curves in $\mathbb{C P}^{2}$ and tubes of radius $\frac{\pi}{2}$ in the first or second normal bundle about a null-torsion pseudoholomorphic curve in $\mathcal{S}^{6}$. We may therefore try to describe the spectrum of the curl operator in these cases.

We begin with the links of complex 2-dimensional cones.

Theorem 6.5. Let $\Sigma$ be a compact, connected, holomorphic curve in $\mathbb{C P}^{2}$ with degree $d_{\Sigma}$. Let $L$ be the Hopf lift of $\Sigma$ in $\mathcal{S}^{5}$. Use the notation of Definitions 4.13 and 6.3. The eigenvalues of $c_{L}$ in $(0,4)$ are $1,2,3$ and $\lambda+2 \in(2,4) \backslash\{3\}$ such that $\lambda(\lambda+2) \in \mathcal{E}_{L}$. Moreover,

$$
\begin{aligned}
& \sigma_{L}(1)=d_{\Sigma}^{2}-d_{\Sigma}, \\
& \sigma_{L}(2)=d_{\Sigma}^{2}+d_{\Sigma}+1, \\
& \sigma_{L}(3)=m_{L}(3)+d_{\Sigma}^{2}+3 d_{\Sigma}, \\
& \sigma_{L}(\lambda+2)=m_{L}(\lambda(\lambda+2)) \text {. }
\end{aligned}
$$

Furthermore, $m_{L}(3) \geq 6$ if $d_{\Sigma} \geq 2$.

Proof. Notice that $L$ is a $\mathrm{U}(1)$-bundle over $\Sigma$, and that the projection $\pi$ : $L \rightarrow \Sigma$ is a Riemannian submersion with totally geodesic fibres when $L$ and $\Sigma$ are given the induced metrics from the standard metrics on $\mathcal{S}^{5}$ and $\mathbb{C P}^{2}$. Therefore, Definition 6.1 and Proposition 6.2 apply. If we let $\xi$ be the vector 
field given by the $\mathrm{U}(1)$ action then, as observed in Example 4.9, $\xi$ is a Killing vector field for the metric on $L$, so Proposition 6.4 applies. Hence, to understand the positive eigenvalues $\nu$ of $c_{L}$, it is enough to study the equations

$$
\begin{aligned}
\mathrm{d} \gamma & =-\nu * \gamma \quad \text { and } \\
\mathcal{L}_{\xi} \gamma & =i m \gamma
\end{aligned}
$$

for $\gamma \in C^{\infty}\left(T^{*} L \otimes \mathbb{C}\right)$ and $m \in \mathbb{Z}$.

Let $\omega_{0}$ be the standard Kähler form on $\mathbb{C}^{3}$. Since $\Sigma$ is holomorphic, $\left.\omega_{0}\right|_{\Sigma}=\operatorname{vol}_{\Sigma}$ and thus $\omega=\left.\omega_{0}\right|_{L}$ is a nowhere vanishing 2 -form on $L$. Moreover, $* \omega$ is the 1 -form on $L$ dual to the $\mathrm{U}(1)$ vector field $\xi$, where $*$ is the Hodge star on $L$, so $\theta=* \omega$ in the notation of Definition 6.1.

By (2.3), if $x_{1}$ is the coordinate on $\mathbb{R}$ in the decomposition $\mathbb{R}^{7}=\mathbb{R} \oplus \mathbb{C}^{3}$ and $C$ is the cone on $L$ embedded in $\mathbb{R}^{7}$, then $\left.\beta=\frac{\partial}{\partial x_{1}}\right\lrcorner\left.\varphi\right|_{C}=\left.\omega_{0}\right|_{C}$ is a selfdual 2-form which corresponds to the deformation of $C$ by translation in the $x_{1}$ direction. By Corollary 2.11 and Proposition 2.17, $\beta$ must be closed. Notice that $\left.\omega_{0}\right|_{C}=r^{2} \omega+r * \omega \wedge \mathrm{d} r$, where $r$ is the radial coordinate, and so $\mathrm{d} \beta=0$ if and only if $\mathrm{d} * \omega=-2 \omega$.

By Definition 6.1, given $\gamma \in C^{\infty}\left(T^{*} L \otimes \mathbb{C}\right)$, there exist $f \in C^{\infty}(L)$ and $\alpha \in C^{\infty}\left(T_{h}^{*} L \otimes \mathbb{C}\right)$ such that $\gamma=f * \omega+\alpha$. Thus,

$$
\mathrm{d} \gamma=\mathrm{d}(f * \omega)+\mathrm{d} \alpha=\mathrm{d}_{h} f \wedge * \omega-2 f \omega+\mathrm{d} \alpha \quad \text { and } \quad * \gamma=f \omega+*_{h} \alpha \wedge * \omega
$$

We deduce from looking at the horizontal components of (6.1) that

$$
\mathrm{d}_{h} \alpha=-(\nu-2) f \omega
$$

For convenience we set $\mathrm{d}_{v}=\mathrm{d}-\mathrm{d}_{h}$. Hence, using Cartan's formula and the facts that $\xi\lrcorner \alpha=\xi\lrcorner \omega=0$ and $\xi\lrcorner * \omega=1,(6.2)$ becomes:

$$
\begin{aligned}
i m \gamma & =\mathrm{d}(\xi\lrcorner \gamma)+\xi\lrcorner \mathrm{d} \gamma=\mathrm{d} f+\xi\lrcorner \mathrm{d}(f * \omega)+\xi\lrcorner \mathrm{d} \alpha \\
& \left.\left.=\mathrm{d} f+\xi\lrcorner\left(\mathrm{d}_{h} f \wedge * \omega\right)+\xi\right\lrcorner \mathrm{d}_{v} \alpha=\mathrm{d}_{v} f+\xi\right\lrcorner \mathrm{d}_{v} \alpha .
\end{aligned}
$$

We can also use (6.1) in (6.2) to see that:

$$
i m \gamma=\mathrm{d} f-\nu \xi\lrcorner * \gamma=\mathrm{d} f-\nu f \xi\lrcorner \omega-\nu \xi\lrcorner\left(*_{h} \alpha \wedge * \omega\right)=\mathrm{d} f+\nu *_{h} \alpha .
$$


Together, we deduce that

$$
\begin{aligned}
\mathrm{d}_{h} f & =i m \alpha-\nu *{ }_{h} \alpha, \\
\mathrm{d}_{v} f & =i m f * \omega, \\
\mathrm{d}_{v} \alpha & =i m * \omega \wedge \alpha .
\end{aligned}
$$

Since $\nu>0,(6.1)$ forces $\mathrm{d}^{*} \gamma=0$ and thus

$$
\mathrm{d}_{h}{ }_{h} \alpha=-i m f \omega .
$$

As in [33, Section 2.2], the complex structure $J$ on $\Sigma$ defines a complex structure map $J_{h}$ on horizontal forms, which agrees with $J$ on horizontal 1 -forms (viewed as lifts of 1 -forms on $\Sigma$ ). This allows us to define $\Lambda_{h}^{p, q} T^{*} L \otimes$ $\mathbb{C}$, where $p, q \in \mathbb{N}$, to be the bundle of horizontal $(p+q)$-forms which have eigenvalue $i(p-q)$ under $J_{h}$, and thus give the decomposition $\Lambda_{h}^{m} T^{*} L \otimes \mathbb{C}=$ $\oplus_{p+q=m} \Lambda_{h}^{p, q} T^{*} L \otimes \mathbb{C}$. We may therefore define operators

$$
\begin{aligned}
& \partial_{h}: C^{\infty}\left(\Lambda_{h}^{p, q} T^{*} L \otimes \mathbb{C}\right) \rightarrow C^{\infty}\left(\Lambda_{h}^{p+1, q} T^{*} L \otimes \mathbb{C}\right) \quad \text { and } \\
& \bar{\partial}_{h}: C^{\infty}\left(\Lambda_{h}^{p, q} T^{*} L \otimes \mathbb{C}\right) \rightarrow C^{\infty}\left(\Lambda_{h}^{p, q+1} T^{*} L \otimes \mathbb{C}\right)
\end{aligned}
$$

by taking appropriate components of $\mathrm{d}_{h}$.

Since $\alpha$ is a horizontal complex 1 -form on $L$, it can be decomposed into $(1,0)$ and $(0,1)$ components. Thus, $*_{h} \alpha=*_{h}\left(\alpha^{1,0}+\alpha^{0,1}\right)=i \alpha^{1,0}-i \alpha^{0,1}$ and so (6.4) becomes:

$$
\begin{aligned}
& \partial_{h} f=i(m-\nu) \alpha^{1,0}, \\
& \bar{\partial}_{h} f=i(m+\nu) \alpha^{0,1} .
\end{aligned}
$$

We notice that $f=0$ forces $m^{2}=\nu^{2}$ otherwise $\alpha=0$ by (6.8) and (6.9). Furthermore, (6.3) for $f=0$ becomes

$$
\bar{\partial}_{h} \alpha^{1,0}+\partial_{h} \alpha^{0,1}=0
$$

Thus, $f=0=m-\nu$ is equivalent to $\bar{\partial}_{h} \alpha^{1,0}=0=\alpha^{0,1}$ and $f=0=m+\nu$ is equivalent to $\partial_{h} \alpha^{0,1}=0=\alpha^{1,0}$.

The hyperplane bundle over $\mathbb{C P}^{2}$ defines a complex line bundle $H$ over $\Sigma$ which is also a real line bundle over $L$. We can, of course, identify $H$ over $L$ with the cone $C$, and so $H$ has a global section $s$ over $L$ given simply by $s(x)=x$. It is clear that $\mathcal{L}_{\xi} s=i s$ and $\alpha \otimes s^{-m}$, by (6.6), is a $\mathrm{U}(1)$-invariant section of $T^{*} L \otimes \mathbb{C} \otimes H^{-m}$, and so pushes down to be a welldefined section of $T^{*} \Sigma \otimes H^{-m}$ over $\Sigma$. The condition $\bar{\partial}_{h} \alpha^{1,0}=0$ given by 
(6.10), when $m=\nu$, is then equivalent to saying that $\alpha^{1,0} \otimes s^{-\nu}$ is a holomorphic section of $P_{\nu}=T^{* 1,0} \Sigma \otimes H^{-\nu}$. Since $H$ is the bundle $\mathcal{O}_{\Sigma}(-1)$ and $T^{* 1,0} \Sigma \cong \mathcal{O}_{\Sigma}\left(d_{\Sigma}-3\right)$, we see that $P_{\nu} \cong \mathcal{O}_{\Sigma}\left(\nu+d_{\Sigma}-3\right)$. Thus, by RiemannRoch, the dimension of the vector space of holomorphic sections of $P_{\nu}$ is

$$
h^{0}\left(P_{\nu}\right)=d_{\Sigma}\left(\nu+d_{\Sigma}-3\right)+1-g_{\Sigma}
$$

(Notice that $P_{3}$ is isomorphic to the normal bundle of $\Sigma$ in $\mathbb{C P}^{2}$ and so its holomorphic sections correspond precisely to the infinitesimal deformations of $\Sigma$ as a holomorphic curve.) Similarly, for $m=-\nu$, we have that $\alpha^{0,1} \otimes$ $s^{\nu}$ is an anti-holomorphic section of $\overline{P_{\nu}}$. Hence, for $f=0$, we get integer eigenvalues $\nu \in\{1,2,3\}$ for $c_{L}$ in $(0,4)$ (since $m^{2}=\nu^{2}$ ) and contributions of $2 h^{0}\left(P_{\nu}\right)$ to $\sigma_{L}(\nu)$. Using the degree-genus formula, we calculate

$$
2 h^{0}\left(P_{\nu}\right)=d_{\Sigma}\left(d_{\Sigma}-3+2 \nu\right) .
$$

It follows from (6.3)-(6.7) that

$$
\begin{aligned}
\Delta_{L} f & =* \mathrm{~d} *\left(i m f * \omega+i m \alpha-\nu *_{h} \alpha\right) \\
& =-* \mathrm{~d}\left(i m f \omega+i m *_{h} \alpha \wedge * \omega+\nu \alpha \wedge * \omega\right) \\
& =-*\left(i m(i m f) \operatorname{vol}_{L}+i m(-i m f) \operatorname{vol}_{L}-\nu(\nu-2) f \operatorname{vol}_{L}\right) \\
& =\nu(\nu-2) f .
\end{aligned}
$$

Since $\mathcal{L}_{\xi} f=i m f$ by $(6.4),(6.5)$, we deduce from Definition 6.1 and Proposition 6.2 that

$$
\Delta_{h} f=\left(\nu(\nu-2)-m^{2}\right) f
$$

Recall that the eigenvalues of $\Delta_{L}$ and $\Delta_{h}$ have to be non-negative.

If $\nu \in(0,2)$ then the only solution of (6.12) is $f=0$. Thus, $m^{2}=\nu^{2}$ by (6.8) and (6.9), so $\nu=1$ and $\sigma_{L}(1)=d_{\Sigma}\left(d_{\Sigma}-1\right)$ from (6.11) as claimed.

If $\nu=2$ and $f \neq 0$ then $m=0$ by (6.13) and the solutions of (6.12) consist of constant (non-zero) functions. Since $f \neq 0$ is constant, we see from (6.8)-(6.9) that $\alpha=0$. Thus, we have a 1 -dimensional space of solutions to (6.1), (6.2) for $\nu=2$ and $f \neq 0$. We deduce the formula for $\sigma_{L}(2)$ from (6.11).

If $\nu \in(2,4)$ and $f \neq 0$ then the non-negativity of $\nu^{2}-2 \nu-m^{2}$ by $(6.13)$ forces $|m| \leq 2$. Moreover, solutions to (6.12) define $\alpha$ via (6.8) and (6.9) since $m^{2} \neq \nu^{2}$. We deduce the remaining eigenvalues of $c_{L}$ and multiplicities from (6.11) as claimed.

Now suppose $d_{\Sigma}>1$ so that the cone $C$ is non-planar. We can view the Lie algebra of $\mathrm{SU}(3)$ as a subalgebra of $\mathfrak{g}_{2}$ and decompose $\mathfrak{g}_{2}=\mathfrak{s u}(3) \oplus$ 
$\mathfrak{s u}(3)^{\perp}$. If $v \in \mathfrak{s u}(3)^{\perp}$ is identified with a tangent vector on $\mathbb{R}^{7},\left.v\right|_{C}$ defines a normal vector $w$ which in turn defines an infinitesimal deformation of $C$ as a coassociative cone. Moreover, since $v \in \mathfrak{s u}(3)^{\perp}$ and $C$ is non-planar, $w$ is a non-trivial infinitesimal deformation which is not complex. Thus $(w\lrcorner \varphi)\left.\right|_{C}$ will be an order $O(r)$ self-dual 2-form, which does not arise purely from a horizontal 1-form on $L$, and will define a 3 -eigenform of $c_{L}$ with $f \neq 0$ (since, for $f=0$, the 3 -eigenforms correspond to the infinitesimal deformations of $\Sigma$ as a holomorphic curve). Since $\operatorname{dim} \mathfrak{s u}(3)^{\perp}=6$, we deduce that $m_{L}(3) \geq 6$ as claimed.

It is easy to check that Theorem 6.5 applied to a totally geodesic $\mathbb{C P}^{1}$ in $\mathbb{C P}^{2}$ implies the stability of complex 2-planes as coassociative 4-planes as we already knew from Corollary 5.7. However, we can in fact prove the following.

Proposition 6.6. The only complex 2-dimensional cones in $\mathbb{C}^{3}$, with compact non-singular complex links in $\mathbb{C P}^{2}$, which are stable as coassociative cones in $\mathbb{R}^{7}$ are complex 2-planes.

Proof. Suppose $C$ is a counterexample to the statement of the proposition and suppose, for simplicity, that the complex link $\Sigma$ of $C$ is connected. Embed $C$ as a coassociative cone in $\mathbb{R}^{7}$ and let $\mathrm{G}$ be the Lie subgroup of $\mathrm{G}_{2}$ preserving $C$. Since $C$ is non-planar and supposed to be stable, in the notation of Proposition 4.12 we should have $\operatorname{dim} \check{D}(0)=7$, since every translation of $C$ will define an order $O(1)$ coassociative deformation, and $\operatorname{dim} \check{D}(1)=14-\operatorname{dim}$ G. By Theorem 6.5 , this occurs if and only if

$$
d_{\Sigma}^{2}+d_{\Sigma}=6 \quad \text { and } \quad d_{\Sigma}^{2}+3 d_{\Sigma}=14-\operatorname{dim} \mathrm{G}-m_{L}(3)
$$

Subtracting these equations and using the fact that $m_{L}(3) \geq 6$ shows that $2 d_{\Sigma} \leq 2-\operatorname{dim} \mathrm{G}$, but this contradicts $d_{\Sigma}>1$ as $C$ is non-planar.

We now wish to mimic our result for holomorphic curves in $\mathbb{C P}^{2}$ for the case of null-torsion pseudoholomorphic curves in $\mathcal{S}^{6}$.

Theorem 6.7. Recall Definitions 4.5, 4.6, 4.13 and 6.3. Let $\Sigma \subseteq \mathcal{S}^{6}$ be a compact, connected, null-torsion pseudoholomorphic curve of genus $g_{\Sigma}$ and let $c_{1}\left(N_{2} \Sigma\right)$ be the first Chern number of $N_{2} \Sigma$. Let $L$ be the tube of radius $\frac{\pi}{2}$ in $N_{2} \Sigma$ about $\Sigma$. The eigenvalues of $c_{L}$ in $(0,4)$ are $1,2,3$ and 
$\lambda+2 \in(2,4) \backslash\{3\}$ such that $\lambda(\lambda+2) \in \mathcal{E}_{L}$. Moreover,

$$
\begin{aligned}
\sigma_{L}(1) & =2 g_{\Sigma}-2 c_{1}\left(N_{2} \Sigma\right)-2, & \sigma_{L}(2) & =2 g_{\Sigma}-4 c_{1}\left(N_{2} \Sigma\right)-1, \\
\sigma_{L}(3) & =m_{L}(3)+2 g_{\Sigma}-6 c_{1}\left(N_{2} \Sigma\right)-2, & \sigma_{L}(\lambda+2) & =m_{L}(\lambda(\lambda+2)) .
\end{aligned}
$$

Proof. It is straightforward to see that Definition 6.1 applies to the natural projection $\pi: L \rightarrow \Sigma$ and that the fibres of $\pi$ are totally geodesic since $L$ is a tube of radius $\frac{\pi}{2}$. Moreover, by Example 4.9, if $\xi$ is the unit vector field given by the $\mathcal{S}^{1}$-fibration of $L$ over $\Sigma$ then $\xi$ is Killing. By Proposition 6.4 it is therefore enough to study (6.1)-(6.2) for $\gamma \in C^{\infty}\left(T^{*} L \otimes \mathbb{C}\right)$ and $m \in \mathbb{Z}$ to find the positive eigenvalues $\nu$ of $c_{L}$.

If we let $\theta$ be the 1 -form on $L$ dual to $\xi$ then, since $\Sigma$ has null-torsion, the structure equations for $L$ given in [28, Section 6.4] imply that $\mathrm{d} \theta=-2 * \theta$. This formula, together with the fact that $\Sigma$ is endowed with a complex structure, enables us to follow the proof of Theorem 6.5 and see that we essentially have two possible contributions to eigenvalues of $c_{L}$ : either eigenfunctions of the Laplacian on $L$ or holomorphic sections of $P_{\nu}=T^{* 1,0} \Sigma \otimes H^{-\nu}$ for $\nu \in \mathbb{Z}^{+}$, where $H=N_{2} \Sigma$ since we can identify the cone over $L$ with $N_{2} \Sigma$ over $\Sigma$.

Recall the structure equations for $\Sigma$ given in Definition 4.6. Since $\kappa_{32}=$ 0 , the curvature form of $N_{2} \Sigma$ is

$$
\mathrm{d} \kappa_{33}=-\theta_{1} \wedge \bar{\theta}_{1},
$$

so $c_{1}\left(N_{2} \Sigma\right)<0$ (in fact, it is proportional to $-\operatorname{vol}(\Sigma)$ ). Thus, by RiemannRoch, we have that

$$
h^{0}\left(P_{\nu}\right)=c_{1}\left(T^{* 1,0} \Sigma\right)-\nu c_{1}\left(N_{2} \Sigma\right)+1-g_{\Sigma}=g_{\Sigma}-1-\nu c_{1}\left(N_{2} \Sigma\right) .
$$

The result now follows from the proof of Theorem 6.5.

\section{Remarks.}

(a) The first Chern number $c_{1}\left(N_{2} \Sigma\right)$ is the negative of the degree of $\Sigma$ as a holomorphic curve in the 5 -quadric in $\mathbb{C P}^{6}$, so is just $-\frac{1}{4 \pi} \operatorname{vol}(\Sigma)$.

(b) Theorem 6.7 will not immediately generalize to the other possible case given by Example 4.9, namely tubes $L$ of radius $\frac{\pi}{2}$ in $N_{1} \Sigma$. First, the relationship between the derivative of the vertical 1-form $\theta$ and its dual $* \theta$ is not as straightforward. Second, $c_{1}\left(N_{1} \Sigma\right)$ is often positive, so it is not as easy to calculate $\sigma_{L}(\nu)$. 
One can apply Theorem 6.7 to the Borůvka sphere $\Sigma$, for which $c_{1}\left(N_{2} \Sigma\right)=$ -6 , and recover the result of Corollary 5.14. Theorem 6.7 can also be applied to a totally geodesic 2-sphere, which is the degenerate case of a null-torsion curve, to prove Corollary 5.7.

We now prove the analogue of Proposition 6.6.

Proposition 6.8. Recall Definitions 4.5 and 4.6. Let $C$ be a coassociative cone whose link is a tube of radius $\frac{\pi}{2}$ in $N_{2} \Sigma$ about a compact (nontotally geodesic) null-torsion pseudoholomorphic curve $\Sigma$ in $\mathcal{S}^{6}$. Then $C$ is not stable.

Proof. Suppose for simplicity that $\Sigma$ is connected and let $\mathrm{G}$ be the subgroup of $\mathrm{G}_{2}$ preserving $C$. By Theorem $6.7, C$ is stable only if

$$
2 g_{\Sigma}-4 c_{1}\left(N_{2} \Sigma\right)=8 \quad \text { and } \quad m_{L}(3)+2 g_{\Sigma}-6 c_{1}\left(N_{2} \Sigma\right)-2=14-\operatorname{dim} \mathrm{G}
$$

since $C$ is non-planar. These equations force

$$
g_{\Sigma}=m_{L}(3)+\operatorname{dim} \mathrm{G}-4 \quad \text { and } \quad 2 c_{1}\left(N_{2} \Sigma\right)=m_{L}(3)+\operatorname{dim} \mathrm{G}-8 .
$$

Therefore, $m_{L}(3)+\operatorname{dim} \mathrm{G} \geq 4$ as $g_{\Sigma} \geq 0$. Hence, $c_{1}\left(N_{2} \Sigma\right) \geq-2$ so the degree of $\Sigma$ as a holomorphic curve in $\mathbb{C P}^{6}$ must be 1 or 2 . We deduce that $\Sigma$ must have genus zero and lie in some $\mathbb{C P}^{2}$ in $\mathbb{C P}^{6}$. We now show that $\Sigma$ cannot be a plane curve by the structure equations in Definition 4.6, giving our required contradiction.

The embedding of $\Sigma$ as a holomorphic curve in $\mathbb{C P}^{6}$ is given by $\mathbf{f}_{3}$. Since $\Sigma$ is non-totally geodesic, $\mathrm{d} \mathbf{f}_{3}$ depends on $\mathbf{f}_{2}$ and $\mathrm{d} \mathbf{f}_{2}$ depends on $\mathbf{f}_{1}$. However, $\mathrm{d} \mathbf{f}_{1}$ has a non-zero component in the direction of $\mathbf{u}$, which is independent of $\mathbf{f}_{1}, \mathbf{f}_{2}, \mathbf{f}_{3}$, since $\theta_{1}$ is nowhere vanishing on the curve. It therefore follows that $\Sigma$ cannot lie in some $\mathbb{C P}^{2}$ in $\mathbb{C P}^{6}$.

Theorems 6.5 and 6.7 invite us to make the following definition.

Definition 6.9. For a compact Riemannian 3-manifold $L$ let

$$
\eta(L)=\sum_{\lambda \in(0,1]} m_{L}(\lambda(\lambda+2)),
$$

using the notation of Definition 6.3.

Remark. By Theorems 6.5 and 6.7, the stability index of certain 2-ruled cones with link $L$ fibred over an algebraic curve $\Sigma$ will be the sum of a 
topological term determined by the degree and genus of $\Sigma$, and an analytic piece given by $\eta(L)$. We can therefore think of $\eta(L)$ as a sort of " $\eta$-invariant".

We now make an elementary observation, which we state for complex cones although it is equally valid for cones whose links are as in Theorem 6.7.

Proposition 6.10. Let $C$ be a complex 2-dimensional cone in $\mathbb{C}^{3}$ with compact real link $L$ such that $C \backslash\{0\}$ is non-singular. Let $\mathcal{C}$ consist of $\mathrm{G}_{2} \ltimes \mathbb{R}^{7}$ transformations of a deformation family for $C$ as a complex cone and recall Definitions 4.12 and 6.9. Let $L^{\prime}$ be the link of $C^{\prime} \in \mathcal{C}$. Then

$$
\operatorname{ind}_{\mathcal{C}}(C)-\operatorname{ind}_{\mathcal{C}}\left(C^{\prime}\right)=\eta(L)-\eta\left(L^{\prime}\right)
$$

for all $C^{\prime} \in \mathcal{C}$. Moreover, there exists an open neighbourhood $\mathcal{C}^{\prime}$ of $C$ in $\mathcal{C}$ such that, for all $C^{\prime} \in \mathcal{C}^{\prime}, \eta(L)-\eta\left(L^{\prime}\right) \geq 0$. Thus, if $C$ is $\mathcal{C}$-stable then $C^{\prime}$ is $\mathcal{C}$-stable for all $C^{\prime} \in \mathcal{C}^{\prime}$.

Proof. By Definitions 4.12 and 4.13 and Theorem 6.5, the difference in the $\mathcal{C}$-stability indices of $C$ and $C^{\prime}$ is determined by the spectra of $L$ and $L^{\prime}$ as claimed because the degree of the complex link is the same for $C$ and $C^{\prime}$.

Deformations of $L$ will change the spectrum, but the only way in which $\eta(L) \neq \eta\left(L^{\prime}\right)$, for a sufficiently small perturbation $L^{\prime}$ of $L$, is if a new element of the spectrum is created strictly above 3 under the deformation and the number of the elements of the spectrum in $(0,3]$ decreases. Thus, $\eta(L) \geq$ $\eta\left(L^{\prime}\right)$ for all $L^{\prime}$ in some open neighbourhood of $L$, proving the existence of $\mathcal{C}^{\prime}$.

If $C$ is $\mathcal{C}$-stable then $\operatorname{ind}_{\mathcal{C}}\left(C^{\prime}\right) \leq \operatorname{ind}_{\mathcal{C}}(C)=0$ for all $C^{\prime} \in \mathcal{C}^{\prime}$. We deduce that $\operatorname{ind}_{\mathcal{C}}\left(C^{\prime}\right)=0$ from the non-negativity of the stability index.

We conclude this section with an application of Proposition 6.10.

Corollary 6.11. Recall $C_{\mathbf{a}}, \mathcal{C}_{\circ}$ and $\mathcal{T}$ defined in Corollary 5.12. The cone $C_{\mathbf{a}}$ is Jacobi integrable and $\mathcal{C}_{\circ}$-stable for all $\mathbf{a} \in \mathcal{T}$.

Proof. Recall that $C_{2}$, with link $L_{2}$, given by Example 4.3 satisfies $C_{2}=$ $C_{\left(\frac{1}{3}, \frac{1}{3}, \frac{1}{3}\right)}$ and $\operatorname{ind}_{\mathcal{C}_{\circ}}\left(C_{2}\right)=0$ by Corollary 5.12 . Let $L_{\mathbf{a}}$ be the link of $C_{\mathbf{a}}$.

Let

$$
\mathcal{T}_{\circ}=\left\{\mathbf{a} \in \mathcal{T}: \operatorname{ind}_{\mathcal{C}_{\circ}}\left(C_{\mathbf{a}}\right)=0\right\}
$$

which is non-empty since $\left(\frac{1}{3}, \frac{1}{3}, \frac{1}{3}\right) \in \mathcal{T}_{\circ}$. By Proposition $6.10 \mathcal{T}_{\circ}$ is open. Our aim is to show that $\mathcal{T}_{\circ}$ is also closed in $\mathcal{T}$, since then $\mathcal{T}_{\circ}=\mathcal{T}$ by connectedness. 
Recall that $\operatorname{dim} \mathcal{C}_{\circ}=23$ and that $m_{L_{\mathbf{a}}}(3) \geq 6$ by Theorem 6.5 . Thus

$$
\operatorname{ind}_{\mathcal{C}_{\circ}}\left(C_{\mathbf{a}}\right)=\sum_{\lambda \in(0,1)} m_{L_{\mathbf{a}}}(\lambda(\lambda+2))+\left(m_{L_{\mathbf{a}}}(3)-6\right)
$$

by Theorem 6.5. We deduce that $\mathbf{a} \in \mathcal{T}_{\circ}$ if and only if $m_{L_{\mathbf{a}}}(3)=6$ and $m_{L_{\mathbf{a}}}(\lambda(\lambda+2))=0$ for all $\lambda \in(0,1)$.

Suppose, for a contradiction, that $\mathcal{T}_{\circ}$ is not closed, so there exists $\mathbf{a} \in$ $\overline{\mathcal{T}_{\circ}} \cap \mathcal{T} \backslash \mathcal{T}_{\circ}$. Therefore $m_{L_{\mathbf{a}}}(3)>6$ or $m_{L_{\mathbf{a}}}(\lambda(\lambda+2))>0$ for some $\lambda \in(0,1)$. However, since the spectrum of the Laplacian varies continuously under deformations of the metric, the latter can occur if and only if there exists $\mathbf{a}^{\prime} \in \overline{\mathcal{T}_{\circ}}$ for which $m_{L_{\mathbf{a}^{\prime}}}(3)>6$. Since this cannot happen for $\mathbf{a}^{\prime} \in \mathcal{T}_{\circ}$ we may suppose therefore that $m_{L_{\mathbf{a}}}(3)>6$.

Hence, there is a Lagrangian Jacobi field $v$ on $L_{\mathbf{a}}$ which is independent of the Lagrangian Jacobi fields corresponding to deformations of $C_{\mathbf{a}}$ in $\mathcal{C}_{\circ}$. For all $\mathbf{a}^{\prime}$ close to $\mathbf{a}$ we have that $L_{\mathbf{a}^{\prime}}=\exp _{v^{\prime}}\left(L_{\mathbf{a}}\right)$ for some Lagrangian Jacobi field $v^{\prime}$. Moreover, since $L_{\mathbf{a}^{\prime}}$ is Lagrangian and $v+v^{\prime}$ is a Lagrangian Jacobi field on $L_{\mathbf{a}}$, we may view $v$ as a Lagrangian Jacobi field on $L_{\mathbf{a}^{\prime}}$ for $\mathbf{a}^{\prime}$ sufficiently close to $\mathbf{a}$. However, any open neighbourhood of a meets $\mathcal{T}_{\circ}$ as $\mathbf{a} \in \overline{\mathcal{T}_{0}}$, so there exists $\mathbf{a}^{\prime} \in \mathcal{T}_{\circ}$ such that $L_{\mathbf{a}^{\prime}}$ has a Lagrangian Jacobi field independent of those corresponding to deformations of $C_{\mathbf{a}^{\prime}}$ in $\mathcal{C}_{\text {o }}$. Thus $m_{L_{\mathbf{a}^{\prime}}}(3)>6$ for some $\mathbf{a}^{\prime} \in \mathcal{T}_{\circ}$, which is our required contradiction.

\section{Examples of coassociative 4-folds with conical singularities}

In this section we produce our examples of coassociative 4-folds with conical singularities. We describe the construction of compact $\mathrm{G}_{2}$ manifolds we require and the singular 4-dimensional submanifolds which arise following [20] and [21]. Initially we will have a singular coassociative 4-fold $N$ in a compact almost $\mathrm{G}_{2}$ manifold $M$, but the ambient $\mathrm{G}_{2}$ structure will have torsion. We then show that $N$ has conical singularities which are stable under deformations of the $\mathrm{G}_{2}$ structure. Finally, we deform the $\mathrm{G}_{2}$ structure on $M$ so that it has no torsion and simultaneously deform $N$ to produce our CS coassociative 4 -fold.

\subsection{Examples of compact $\mathrm{G}_{2}$ manifolds}

Here we review the relevant material from [20]. The key ingredients will be Fano 3-folds and K3 surfaces, which we now define. 
Definition 7.1. A compact complex 3-dimensional manifold $X$ is a Fano 3-fold if its first Chern class is positive. Equivalently, $X$ has ample anticanonical bundle. Fano 3 -folds are simply connected and projective.

A $K 3$ surface $P$ is a simply connected, compact, complex surface with $c_{1}(P)=0$. A generic divisor in the anticanonical linear system of a Fano 3 -fold is a smooth $K 3$ surface by the work of Shokurov [36].

Remark. One definition of a Calabi-Yau 3-fold is a compact Kähler 3-fold with vanishing first Chern class or, equivalently, with trivial canonical bundle.

The construction of compact $\mathrm{G}_{2}$ manifolds in [20] proceeds via the construction of certain non-compact Calabi-Yau 3-folds. These non-compact Riemannian manifolds are asymptotically cylindrical. We define these manifolds formally.

Definition 7.2. Let $(Y, g)$ be a Riemannian $n$-manifold. We say that $Y$ is asymptotically cylindrical (with rate $\lambda$ ) if there exist constants $\lambda<0$ and $R>0$, a compact subset $K$ of $Y$, a compact Riemannian $(n-1)$-manifold $\left(S, g_{S}\right)$ and a diffeomorphism $\Psi:(R, \infty) \times S \rightarrow Y \backslash K$ satisfying

$$
\left|\nabla^{j}\left(\Psi^{*}(g)-g_{\text {cyl }}\right)\right|=O\left(e^{\lambda t}\right), \quad \text { as } t \rightarrow \infty \text { for all } j \in \mathbb{N}
$$

where $g_{\text {cyl }}=\mathrm{d} t^{2}+g_{S}$ is the cylindrical metric on $(0, \infty) \times S, \nabla$ is the Levi-Civita connection of $g_{\text {cyl }}$ and |.| is calculated with respect to $g_{\text {cyl }}$.

We now introduce some important notation.

Definition 7.3. Let $\mathcal{X}$ be a maximal deformation family of Fano 3-folds, let $X \in \mathcal{X}$ and let $P, Q$ be $K 3$ surfaces in the anticanonical linear system of $X$ such that $P \cap Q$ is a non-singular curve in $X$. Let $\tilde{X}(P, Q)$ denote the blow-up of $X$ along $P \cap Q$ and let $\tilde{P}$ denote the proper transform of $P$ in $\tilde{X}(P, Q)$. Finally, let $Y(X, P, Q)=\tilde{X}(P, Q) \backslash \tilde{P}$.

Since $\tilde{X}(P, Q)$ is the blow-up of $X$ along $P \cap Q$, we have a smooth map $\varpi: \tilde{X}(P, Q) \rightarrow \mathbb{C P}^{1}$ whose fibres are the proper transforms of the divisors in the pencil defined by $P$ and $Q$. We may introduce a holomorphic coordinate $\zeta$ on $\mathbb{C P}^{1}$ such that $\varpi^{-1}(0)=\tilde{P}$ and see that, for some open neighbourhood $U$ of 0 in the $\zeta$ coordinate, $\varpi^{-1}(U \backslash\{0\})$ is diffeomorphic to $(0, \infty) \times P \times \mathcal{S}^{1}$. Thus we may view $Y(X, P, Q)$ as a manifold with a cylindrical "end" with cross-section $P \times \mathcal{S}^{1}$. This motivates the next result which follows from [20, Corollary 6.43]. 
Theorem 7.4. Use the notation of Definition 7.3. There is a smooth complete metric $g_{Y}$ on $Y=Y(X, P, Q)$ such that $\left(Y, g_{Y}\right)$ is asymptotically cylindrical and the holonomy of $g_{Y}$ is $\mathrm{SU}(3)$.

Suppose we have a pair of maximal deformation families $\mathcal{X}_{1}$ and $\mathcal{X}_{2}$ of Fano 3-folds. Using the notation of Definition 7.3, we have a pair of asymptotically cylindrical complex 3 -folds $Y_{1}=Y_{1}\left(X_{1}, P_{1}, Q_{1}\right)$ and $Y_{2}=$ $Y_{2}\left(X_{2}, P_{2}, Q_{2}\right)$ with holonomy $\mathrm{SU}(3)$ by Theorem 7.4. We thus have 7-manifolds $Z_{i}=Y_{i} \times \mathcal{S}^{1}$, for $i=1,2$, which are asymptotically cylindrical to $(0, \infty) \times P_{i} \times \mathcal{S}^{1} \times \mathcal{S}^{1}$. In [20, Section 4] it is explained that if $P_{1}$ and $P_{2}$ satisfy a certain "matching condition", then one can apply a "twisted connected sum" construction to $Z_{1}$ and $Z_{2}$ to get a one-parameter family of compact almost $\mathrm{G}_{2}$ manifolds $\left\{\left(M_{T}, \varphi_{T}, g_{\varphi_{T}}\right): T>T_{0}\right\}$ for some $T_{0}>0$. Moreover, $\left(\varphi_{T}, g_{\varphi_{T}}\right)$ is simply the product $\mathrm{G}_{2}$ structure on $Z_{i}$, as described in Definition 2.18, away from the "interpolation region" where $Z_{1}$ and $Z_{2}$ are "glued". The only question is whether this "matching condition" holds for $P_{1}$ and $P_{2}$. The answer [20, Theorem 6.44] is that there always exist $X_{i} \in \mathcal{X}_{i}$ such that $P_{1}$ and $P_{2}$ can be chosen which satisfy the "matching condition". Finally, [20, Proposition 5.32 and Theorem 5.34] imply that one can always perturb the closed $\mathrm{G}_{2}$ structure on $M_{T}$ to a torsion-free one for sufficiently large $T$. We can summarize these observations as a theorem.

Theorem 7.5. Let $\mathcal{X}_{1}$ and $\mathcal{X}_{2}$ be maximal deformation families of Fano 3-folds and recall the notation of Definitions 2.13, 2.14, 2.15 and 7.3. There exist constants $T_{0}>0$ and $\lambda<0$ and, for $i=1,2, X_{i} \in \mathcal{X}_{i}$ and a K3 surface $P_{i}$ in the anticanonical linear system of $X_{i}$ such that, for all $T>T_{0}$ and suitable $Q_{1}, Q_{2}$ as in Definition 7.3, the following hold.

(a) There is a compact almost $\mathrm{G}_{2}$ manifold $\left(M_{T}, \varphi_{T}, g_{\varphi_{T}}\right)$ which, outside some compact set $I_{T}$, is diffeomorphic to the disjoint union of $Y_{1}\left(X_{1}, P_{1}, Q_{1}\right) \times \mathcal{S}^{1}$ and $Y_{2}\left(X_{2}, P_{2}, Q_{2}\right) \times \mathcal{S}^{1}$ endowed with the product $\mathrm{G}_{2}$ structure induced from the asymptotically cylindrical $\mathrm{SU}(3)$ structure given by Theorem 7.4.

(b) Let $p>4$. There is a smooth 2-form $\eta_{T}$ on $M_{T}$, satisfying $\left\|\eta_{T}\right\|_{L_{2}^{p}} \leq$ $c_{p} e^{\lambda T}$ and $\left\|\eta_{T}\right\|_{C^{1}} \leq c_{p} e^{\lambda T}$ for some constant $c_{p}>0$, such that $\varphi_{T}+$ $\mathrm{d} \eta_{T} \in C^{\infty}\left(\Lambda_{+}^{3} T^{*} M_{T}\right)$ and the metric $g_{\varphi_{T}+\mathrm{d} \eta_{T}}$ on $M_{T}$ has holonomy $\mathrm{G}_{2}$.

The compact set $I_{T}$ is the interpolation region between $Z_{1}$ and $Z_{2}$. 
Note. Of course, the $M_{T}$ are topologically the same for all $T$, so we can view $\left(\varphi_{T}, g_{\varphi_{T}}\right)$ as a one-parameter family of closed $\mathrm{G}_{2}$ structures on a 7-manifold $M$.

We now have the following important result.

Proposition 7.6. Use the notation of Theorem 7.5. Outside $I_{T}, M_{T}$ is fibred by K3 surfaces which are coassociative with respect to $\varphi_{T}$. Moreover, for generic $Q_{1}$ and $Q_{2}$, there exist coassociative $K 3$ surfaces in $\left(M_{T}, \varphi_{T}, g_{\varphi_{T}}\right)$ whose singularities are isolated and are ordinary double points.

Proof. After Definition 7.3 we noted that we have a fibration $\varpi: \tilde{X}(P, Q) \rightarrow$ $\mathbb{C P}^{1}$ whose fibres are $K 3$ surfaces. Necessarily some of these fibres will be singular and the generic singularity is an ordinary double point. Recall that we have the freedom to choose any smooth $K 3$ surface $Q$ in the anticanonical linear system of $X$ which meets $P$ in a non-singular curve. Therefore, through generic choice of $Q$ we can be assured that there are fibres other than the exceptional divisor whose only singularities are ordinary double points. Thus, for generic $Q_{1}$ and $Q_{2}$, we have a fibration

$$
\varpi_{T}: M_{T} \backslash I_{T} \cong\left(Y_{1} \times \mathcal{S}^{1}\right) \sqcup\left(Y_{2} \times \mathcal{S}^{1}\right) \rightarrow\left(\mathbb{C P}^{1} \sqcup \mathbb{C P}^{1}\right) \times \mathcal{S}^{1}
$$

with fibres that are $K 3$ surfaces in $Y_{i} \times\{x\}$ for some $i$ and some $x \in \mathcal{S}^{1}$. Moreover, there are some fibres of $\varpi_{T}$ which only have ordinary double point singularities. Since $Y_{1}$ and $Y_{2}$ are Calabi-Yau manifolds and the almost $\mathrm{G}_{2}$ structure on $M_{T} \backslash I_{T}$ agrees with the product $\mathrm{G}_{2}$ structure on $Y_{1} \times \mathcal{S}^{1}$ and $Y_{2} \times \mathcal{S}^{1}$, a simple generalization of Corollary 2.11 leads us to deduce that the fibres of $\varpi_{T}$ are coassociative with respect to $\varphi_{T}$.

Remark. By studying the $\mathrm{G}_{2}$ structure on $I_{T}$, it is shown in [21] that one can extend the coassociative fibration $\varpi_{T}$ through $I_{T}$.

For convenience we introduce the following notation.

Definition 7.7. Use the notation of Theorem 7.5 and let $Q_{1}$ and $Q_{2}$ be generic so that Proposition 7.6 applies. Let $\Gamma_{T}$ denote the set of coassociative $K 3$ surfaces in $\left(M_{T}, \varphi_{T}, g_{\varphi_{T}}\right)$, which have isolated ordinary double point singularities. By Proposition 7.6, there exist $N \in \Gamma_{T}$ such that $N \subseteq M_{T} \backslash I_{T}$.

Our aim now is to show that some of the singular coassociative 4-folds in $\Gamma_{T}$ are "stable" under the deformation from the closed $\mathrm{G}_{2}$ structure $\varphi_{T}$ to the torsion-free $\mathrm{G}_{2}$ structure $\varphi_{T}+\mathrm{d} \eta_{T}$ given in Theorem 7.5(b). 


\subsection{Stable coassociative conical singularities}

We begin with a crucial result that allows us to implement our stability theory.

Proposition 7.8. Use the notation of Theorem 7.5 and Definition 7.7. If $N \in \Gamma_{T}$, then $N$ is a CS coassociative 4-fold in $\left(M_{T}, \varphi_{T}, g_{\varphi_{T}}\right)$ in the sense of Definition 3.2. Moreover, the singularities of $N$ have cones in the family $\mathcal{C}_{\circ}$ given in Corollary 5.12.

Proof. First observe that $N$ is clearly a connected coassociative integral current with $\partial N=\emptyset$. Second, if $z$ is a singular point of $N$ then it is an ordinary double point of a complex surface, so the tangent cone at $z$ has multiplicity one and is modelled on a cone in $\mathcal{C}_{\circ}$ by definition. Since cones in $\mathcal{C}_{\circ}$ are Jacobi integrable by Corollary 6.11 , we may apply Corollary 3.20 to deduce the result.

Proposition 7.9. Let $N$ be a CS coassociative 4-fold in an almost $\mathrm{G}_{2}$ manifold. Suppose that the singularities of $N$ are $z_{1}, \ldots, z_{s}$ with rate $\mu$ and cones $C_{1}, \ldots, C_{s}$ such that:

(i) $C_{i}$ is in the family $\mathcal{C}_{\circ}$ given in Corollary 5.12 for all $i$; and

(ii) $(1, \mu] \cap \mathcal{D}=\emptyset$, where $\mathcal{D}$ is given in Definition 3.12.

If $\mathfrak{C}=\mathcal{C}_{\circ}^{s}$, then $\mathcal{O}(N, \mu, \mathfrak{C})=\{0\}$, in the notation of Theorem 3.14.

Proof. This follows immediately from Definition 4.12 and Corollary 6.11.

Theorem 1.3 now follows from our final result.

Theorem 7.10. Use the notation of Theorem 7.5 and Definition 7.\%. Let $T>T_{0}$ and let $N \in \Gamma_{T}$ be such that $N \subseteq M_{T} \backslash I_{T}$. Making $T_{0}$ larger if necessary, there exists a CS deformation $N^{\prime}$ of $N$ which is coassociative with respect to $\varphi_{T}+\mathrm{d} \eta_{T}$.

Proof. Note that there are $N \in \Gamma_{T}$ which do not lie in $I_{T}$ and that we are free to make the rate $\mu$ at the singularities of $N$ lower if necessary to satisfy $(1, \mu] \cap \mathcal{D}=\emptyset$. Hence, Propositions 7.8 and 7.9 imply $N$ is a CS coassociative 4-fold with respect to $\varphi_{T}$ and that $\mathcal{O}(N, \mu, \mathfrak{C})=\{0\}$.

Recall that $M_{T^{\prime}}$ is diffeomorphic to some 7 -manifold $M$ for all $T^{\prime} \geq$ $T_{0}$. Let $\mathcal{F}=\left\{\left(\varphi_{t}+s \mathrm{~d} \eta_{t}, g_{\varphi_{t}+s \mathrm{~d} \eta_{t}}\right): s \in \mathbb{R}, t>T_{0}\right\}$. Then $\mathcal{F}$ is a smooth 
2-dimensional family of closed $\mathrm{G}_{2}$ structures on $M$. Moreover, for each $T>T_{0}$ we may parameterize $\mathcal{F}$ by $(u, v) \in B(0 ; 1)$ via

$$
(u, v) \in B(0 ; 1) \subseteq \mathbb{R}^{2} \mapsto \varphi^{(u, v)}=\varphi_{t_{T}(u, v)}+s_{T}(u, v) \mathrm{d} \eta_{t_{T}(u, v)},
$$

where

$$
s_{T}(u, v)=\frac{\left(T-T_{0}\right) v}{(1-u)^{2}+v^{2}}
$$

and

$$
t_{T}(u, v)=\frac{T_{0}(1-u)^{2}+T_{0} v^{2}+\left(T-T_{0}\right)(1-u)}{(1-u)^{2}+v^{2}}
$$

With this parameterization of $\mathcal{F}$ we see that $\varphi^{(0,0)}=\varphi_{T}$.

Notice that, since $N \cap I_{T}=\emptyset$ there exists $\tau>0$ such that $T>T_{0}+\tau$ and $N \cap I_{T^{\prime}}=\emptyset$ for all $T^{\prime} \in(T-\tau, T+\tau)$. As the $\mathrm{G}_{2}$ structure outside $I_{T^{\prime}}$ is the same for all $T^{\prime} \in(T-\tau, T+\tau)$ by Theorem 7.5(a), $N$ is coassociative with respect to $\varphi_{T^{\prime}}$ for all such $T^{\prime}$. We therefore see that $\left[\varphi^{(u, v)}\right]=0$ in $H_{\mathrm{cs}}^{3}(\hat{N})$ for all $(u, v)$ sufficiently near $(0,0)$. Hence, by Theorem 3.17 , there exists some $\delta_{N}>0$ such that for all $(u, v) \in B\left(0 ; \delta_{N}\right)$ there exists a CS deformation $N^{(u, v)}$ of $N$ which is coassociative with respect to $\varphi^{(u, v)}$.

To complete the proof, we need to show that $\varphi^{(u, v)}=\varphi_{T}+\mathrm{d} \eta_{T}$ for some $(u, v) \in B\left(0 ; \delta_{N}\right)$. As discussed after Theorem 3.17, this occurs if $\left\|\mathrm{d} \eta_{T}\right\|_{C^{1}}<$ $\epsilon$ and $\left\|\mathrm{d} \eta_{T}\right\|_{L_{2}^{p}}<\epsilon$ for some $p>4$, where $\epsilon>0$ is determined by the geometry near $N$ with respect to $g_{\varphi_{T}}$. By Theorem $7.5(\mathrm{~b}),\left\|\mathrm{d} \eta_{T}\right\|_{C^{1}}$ and $\left\|\mathrm{d} \eta_{T}\right\|_{L_{2}^{p}}$ are of order $O\left(e^{\lambda T}\right)$ for some $\lambda<0$. Moreover, by Theorem 7.5(a), the geometry near $N$ is not changing as $T$ varies, so the constant $\epsilon$ can be chosen to be independent of $T$. Thus, we can ensure that the relevant norms of $\mathrm{d} \eta_{T}$ are sufficiently small by making $T_{0}$ larger.

\section{Acknowledgements}

The author particularly thanks Robert Bryant, Dominic Joyce and Alexei Kovalev for useful conversations, and University College, Oxford, for hospitality during the course of this project. The author also thanks the referee for helpful comments and suggestions. The author is currently supported by an EPSRC Career Acceleration Fellowship. 


\section{References}

[1] D. Adams and L. Simon, Rates of asymptotic convergence near isolated singularities of geometric extrema, Indiana Univ. Math. J. 37 (1988), $225-254$.

[2] R. Bartnik, The mass of an asymptotically flat manifold, Comm. Pure Appl. Math. 39 (1986), 661-693.

[3] L. Bérard Bergery and J.-P. Bourguignon, Laplacians and Riemannian submersions with totally geodesic fibres, Illinois J. Math. 26 (1982), 181-200.

[4] R.L. Bryant, Submanifolds and special structures on the octonions, J. Diff. Geom. 17 (1982), 185-232.

[5] R.L. Bryant, Some remarks on $\mathrm{G}_{2}$-structures, Proceedings of Gökova Geometry-Topology Conference 2005, eds. S. Akbulut, T. Önder and R.J. Stern, International Press, 2006.

[6] A. Corti, M. Haskins, J. Nordström and T. Pacini, G ${ }_{2}$ manifolds and associative submanifolds via semi-Fano 3-folds, Preprint, arXiv:1207.4470.

[7] F. Dillen, L. Verstraelen and L. Vrancken, Classification of totally real 3dimensional submanifolds of $\mathcal{S}^{6}(1)$ with $K \geq 1 / 16$, J. Math. Soc. Japan 42 (1990), 565-584.

[8] J. Etnyre and R. Ghrist, Contact topology and hydrodynamics. I. Beltrami fields and the Seifert conjecture, Nonlinearity 13 (2000), 441-458.

[9] D. Fox, Coassociative cones that are ruled by 2-planes, Asian J. Math. 11 (2007), 535-554.

[10] R. Harvey and H.B. Lawson, Calibrated geometries, Acta Math. 148 (1982), 47-152.

[11] M. Haskins, The geometric complexity of special Lagrangian $T^{2}$-cones, Invent. Math. 157 (2004), 11-70.

[12] A. Ikeda, On the spectrum of homogeneous spherical space forms, Kodai Math. J. 18 (1995), 57-67.

[13] D.D. Joyce, Compact manifolds with special holonomy, OUP, Oxford, 2000 . 
[14] D.D. Joyce, Special Lagrangian submanifolds with isolated conical singularities. V. Survey and applications, J. Diff. Geom. 63 (2003), 299-347.

[15] D.D. Joyce, Special Lagrangian submanifolds with isolated conical singularities. I. Regularity, Ann. Global Ann. Geom. 25 (2004), 201-251.

[16] D.D. Joyce, Special Lagrangian submanifolds with isolated conical singularities. II. Moduli spaces, Ann. Global Ann. Geom. 25 (2004), 301-352.

[17] D.D. Joyce, Special Lagrangian submanifolds with isolated conical singularities. III. Desingularization, the unobstructed case, Ann. Global Ann. Geom. 26 (2004), 1-58.

[18] D.D. Joyce, Special Lagrangian submanifolds with isolated conical singularities. IV. Desingularization, obstructions and families, Ann. Global Ann. Geom. 26 (2004), 117-174.

[19] D.D. Joyce, Riemannian holonomy groups and calibrated geometry, Oxford Graduate Texts in Mathematics 12, OUP, Oxford, 2007.

[20] A.G. Kovalev, Twisted connected sums and special Riemannian holonomy, J. Reine Angew. Math. 565 (2003), 125-160.

[21] A.G. Kovalev, Coassociative K3 fibrations of compact $\mathrm{G}_{2}$-manifolds, Preprint, arXiv:math/0511150.

[22] H.B. Lawson and R. Osserman, Non-existence, non-uniqueness and irregularity of solutions to the minimal surface system, Acta Math. 139 (1977), 1-17.

[23] R.B. Lockhart and R.C. McOwen, Elliptic differential operators on noncompact manifolds, Ann. Sc. Norm. Super. Pisa Cl. Sci. 12 (1985), 409447 .

[24] J.D. Lotay, 2-Ruled calibrated 4-folds in $\mathbb{R}^{7}$ and $\mathbb{R}^{8}$, J. London Math. Soc. 74 (2006), 219-243.

[25] J.D. Lotay, Coassociative 4-folds with conical singularities, Comm. Anal. Geom. 15 (2007), 891-946.

[26] J.D. Lotay, Desingularization of coassociative 4-folds with conical singularities, Geom. Funct. Anal. 18 (2008), 2055-2100.

[27] J.D. Lotay, Deformation theory of asymptotically conical coassociative 4-folds, Proc. London Math. Soc. 99 (2009), 386-424. 
[28] J.D. Lotay, Ruled Lagrangian submanifolds of the 6-sphere, Trans. Amer. Math. Soc. 363 (2011), 2305-2339.

[29] K. Mashimo, Homogeneous totally real submanifolds of $\mathcal{S}^{6}$, Tsukuba J. Math. 9 (1985), 185-202.

[30] V.G. Maz'ya and B. Plamenevskij, Elliptic boundary value problems, Amer. Math. Soc. Transl. 123 (1984), 1-56.

[31] R.C. McLean, Deformations of calibrated submanifolds, Comm. Anal. Geom. 6 (1998), 705-747.

[32] F. Morgan, Geometric measure theory: a beginner's guide, 4th edition, Academic Press, San Diego, 2009.

[33] P.-A. Nagy, Un principe de séparation des variables pour le spectre du Laplacien des formes differentielles et applications, $\mathrm{PhD}$ thesis, Université de Savoie, 2001.

[34] Y. Ohnita, Stability and rigidity of special Lagrangian cones over certain minimal Legendrian orbits, Osaka J. Math. 44 (2007), 305-334.

[35] S. Salamon, Riemannian geometry and holonomy groups, Pitman Research Notes in Math. 201, Longman, Harlow, 1989.

[36] V.V. Shokurov, Smoothness of a general anticanonical divisor on a Fano variety, Izv. Akad. Nauk SSSR Ser. Mat. 43 (1979), 430-441; English transl.: Math. USSR Izb 14 (1980), 395-405.

[37] L. Simon, Isolated singularities of extrema of geometric variational problems, in Harmonic mappings and minimal immersions, ed. E. Giusti, Lect. Notes Math. 1161, Springer-Verlag, Berlin, 1985, 206-277.

[38] A. Strominger, S.T. Yau and E. Zaslow, Mirror symmetry is T-duality, Nucl. Phys. B 479 (1996), 243-259.

[39] S. Tanno, The first eigenvalue of the Laplacian on spheres, Tôhoku Math. J. 31 (1979), 179-185.

[40] H. Urakawa, On the least eigenvalue of the Laplacian for compact group manifolds, J. Math. Soc. Japan 31 (1979), 209-226.

[41] L. Vrancken, Killing vector fields and Lagrangian submanifolds of the nearly Kähler $\mathcal{S}^{6}$, J. Math. Pures Appl. 77 (1998), 631-645. 
[42] B. Watson, Manifold maps commuting with the Laplacian, J. Diff. Geom. 8 (1973), 85-94.

Department of Mathematics

University COLLEge LONDON

GOWER STREeT

LONDON WC1E 6BT

ENGLAND, UK

E-mail address: j.lotay@ucl.ac.uk

Received January 6, 2011 
